\title{
NEW DIRECTIONS IN CATEGORICAL LOGIC, FOR CLASSICAL, PROBABILISTIC AND QUANTUM LOGIC
}

\author{
BART JACOBS
}

Institute for Computing and Information Sciences, Radboud University Nijmegen, The Netherlands.

$U R L:$ www.cs.ru.nl/B. Jacobs

e-mail address: bart@cs.ru.nl

\begin{abstract}
Intuitionistic logic, in which the double negation law $\neg \neg P=P$ fails, is dominant in categorical logic, notably in topos theory. This paper follows a different direction in which double negation does hold, especially in quantitative logics for probabilistic and quantum systems. The algebraic notions of effect algebra and effect module that emerged in theoretical physics form the cornerstone. It is shown that under mild conditions on a category, its maps of the form $X \rightarrow 1+1$ carry such effect module structure, and can be used as predicates. Maps of this form $X \rightarrow 1+1$ are identified in many different situations, and capture for instance ordinary subsets, fuzzy predicates in a probabilistic setting, idempotents in a ring, and effects (positive elements below the unit) in a $C^{*}$-algebra or Hilbert space.

In quantum foundations the duality between states and effects (predicates) plays an important role. This duality appears in the form of an adjunction in our categorical setting, where we use maps $1 \rightarrow X$ as states. For such a state $\omega$ and a predicate $p$, the validity probability $\omega \models p$ is defined, as an abstract Born rule. It captures many forms of (Boolean or probabilistic) validity known from the literature.

Measurement from quantum mechanics is formalised categorically in terms of 'instruments', using Lüders rule in the quantum case. These instruments are special maps associated with predicates (more generally, with tests), which perform the act of measurement and may have a side-effect that disturbs the system under observation. This abstract description of side-effects is one of the main achievements of the current approach. It is shown that in the special case of $C^{*}$-algebras, side-effects appear exclusively in the noncommutative (properly quantum) case. Also, these instruments are used for test operators in a dynamic logic that can be used for reasoning about quantum programs/protocols.

The paper describes four successive assumptions, towards a categorical axiomatisation of quantitative logic for probabilistic and quantum systems, in which the above mentioned elements occur.
\end{abstract}

\section{INTRODUCTION}

Mathematical logic started in the 19th century with George Boole's Laws of Thought. Since then 'Boolean algebras' form the (algebraic) basis of formal logic. In the 20th century these

2012 ACM CCS: [Theory of computation]: Models of computation-Probabilistic computation / Quantum computation theory; Semantics and reasoning-Program semanticsCategorical semantics.

Key words and phrases: quantum logic, measurement, categorical logic, effect algebra, side-effects. 


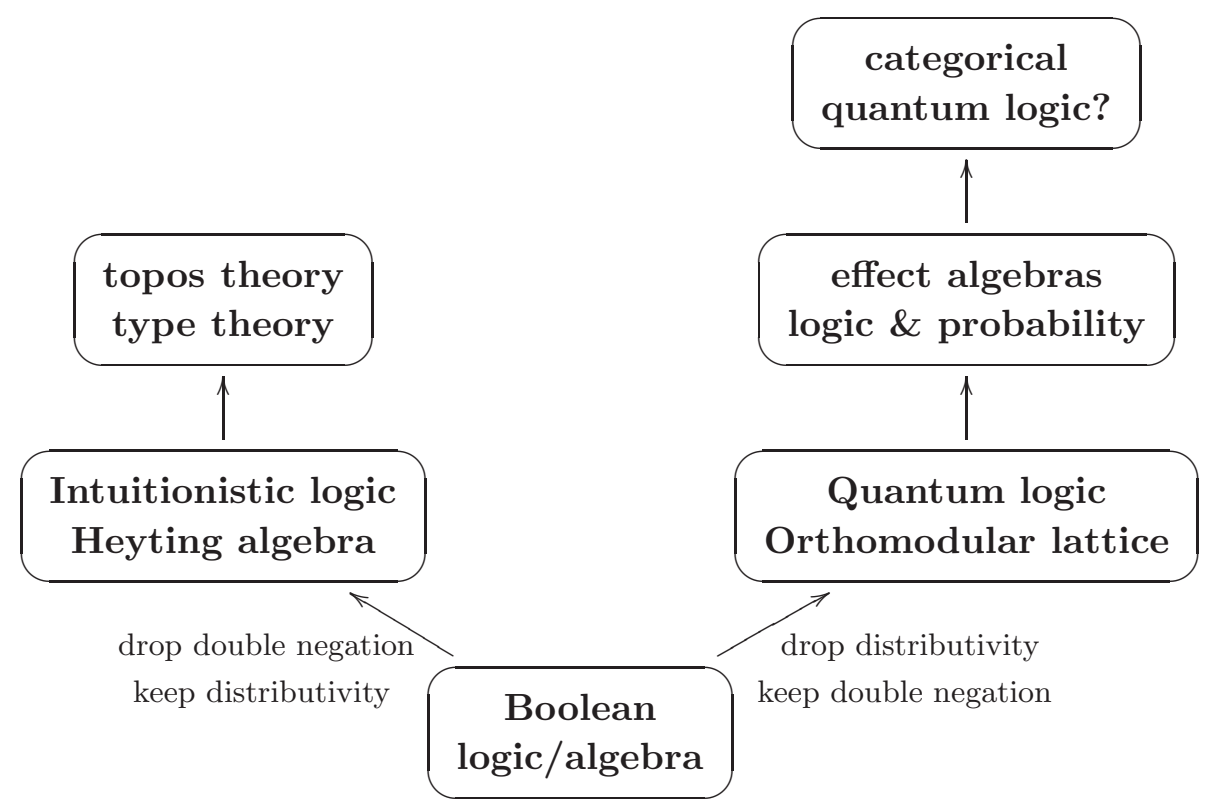

Figure 1: Generalisations of Boolean logic

Boolean algebras have been generalised, mainly in two directions, see Figure 1) Of these two directions the one on the left is most familiar and dominant. It has given rise to the edifice of topos theory, where the logic naturally associated with geometric structures is intuitionistic - in the sense that the double-negation law $\neg \neg P=P$ fails. Also, it has given rise to Martin-Löf's intuitionistic type theory [59, 65], which forms the foundation for the theorem prover Coq (and other such computerised formal systems). In this tradition it is claimed that the natural logic of computation is intuitionistic.

However, there are generalisations of Boolean logic where double-negation does hold, like linear logic or quantum logic. In this tradition negation is often written as superscript $P^{\perp}$ and called orthocomplement. The logic of the quantum world has first been formalised by von Neumann and Birkhoff in terms of orthomodular lattices [64, 8] (see also the monograph [50]). But these orthomodular lattices are rather awkward mathematical structures that have not led to a formal calculus of propositions (or predicates) capturing relevant logical phenomena in physics.

A second generalisation, started in the 1990s, involves so-called effect algebras [25] and effect modules, introduced in various forms (e.g. as D-posets [18, or as weak orthoalgebras in [29]) and by various people (see [24]), notably by theoretical physicists Foulis, Bennet, Gudder and Pulmannová, who published their work in the physics literature, largely outside the range of perception of traditional logicians. These effect algebras are partial commutative monoids with an orthocomplement $(-)^{\perp}$. Examples come from classical logic (Boolean algebras), quantum logic in a Hilbert space or $C^{*}$-algebra, probability theory (the unit interval $[0,1]$ ) and measure theory (step functions, taking finitely many values). Effect algebras form a common generalisation of structures from logic and probability theory, making it possible to see both negation $P \mapsto \neg P$ in logic and opposite probability $r \mapsto 1-r$ as instances of the same orthocomplement operation. Partial commutative monoids have 
appeared in programming language semantics early on, notably in the partially additive structures of [5], and also in models of linear logic [33. But orthocomplement is typical of the more restrictive quantum context.

As an aside: the name 'effect' in 'effect algebra' refers to the observer effect 11 that may arise in a quantum setting, where the act of observation may disturb the system under observation, see [57, 11, 10]. There is no historical connection with the use of the word 'effect' in the context of computer programming with monads (going back to 62]). However, there is a similarity in meaning. Both usages of the word 'effect' - in physics and in computer science - refer to a 'side-effect', that is, to a change in an underlying state space.

Effect modules are effect algebras with an additional scalar multiplication, where the scalars are typically taken from the unit interval $[0,1]$. Intuitively, these effect modules can be seen as vector spaces, defined not over the real or complex numbers, but over $[0,1]$. They are the algebraic counterparts of quantitative logics, where predicates can be multiplied with a scaling factor. This includes fuzzy predicates and effects in Hilbert spaces or $C^{*}$-algebras. In fact, we present a general construction for effect modules of predicates in a category, see Proposition 4.6. This forms an important part of our development of the right leg in Figure 1

The basic theory of effect algebras and modules has been developed by several physicists who (understandably) lacked a background in modern logic, (program) semantics, and categorical logic. Especially the unfamiliarity with the modern methods of categorical logic seriously hampered, or even blocked, progress in this field. As a result these founders failed to identify certain fundamental relationships and similarities with programming semantics and logic (notably the categorical dualities between convex sets and effect modules [44, described in quantum theoretic terms as the duality between states and effects, or between Schrödinger's and Heisenberg's view, see [35, Chapter 2]). Here this duality is described in terms of an adjunction $\mathbf{E M o d}^{\mathrm{op}} \rightleftarrows$ Conv between categories of effect modules and convex sets, see Figure 3 and Proposition 2.6 .

One of the great contributions in the left leg of Figure 1 is the notion of a topos (see e.g. 61]), which can be seen as a categorical formulation of intuitionistic set theory and logic. One wonders: is there a comparably important (categorical) notion in the right leg? Is there an analogue of a topos for the quantum world, incorporating a quantitative logic? This question forms the main motivation for the research presented in this paper. We do not claim to have the definitive answer at this stage, but we do think that we identify some key properties of such an analogue, belonging to the right leg in Figure 1.

In topos theory predicates on an object $X$ can equivalently be described as subobjects of $X$ and as maps $X \rightarrow \Omega$, where $\Omega$ is a special object called the subobject classifier. This $\Omega$ carries the structure of a Heyting algebra. Here we shall use maps of the form $p: X \rightarrow 1+1$ as predicates, where 1 is the final object and + is coproduct. Such predicates have a classical flavour, since there is an associated orthocomplement $p^{\perp}$ obtained by swapping the output options. We write this as $p^{\perp}=\left[\kappa_{2}, \kappa_{1}\right] \circ p$, where the $\kappa_{i}: 1 \rightarrow 1+1$ are coprojections and $[-,-]$ is cotupling. Double negation $p^{\perp \perp}=p$ holds by construction.

In the ordinary set-theoretic world there is a one-to-one correspondence between subsets $S \subseteq X$ of a set $X$ and characteristic functions $X \rightarrow 1+1$ on $X$. Both these notions can be used as interpretation of predicates. This one-to-one correspondence fails in many other settings. One can say that the emphasis in the left leg in Figure 1 lies on the 'spatial'

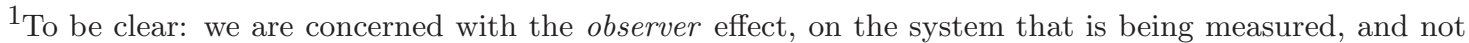
with the possible effect on the measurement apparatus.
} 
interpretation of predicates as subsets. The path on the right in Figure 1 rests, so we claim, on the view of predicates as characteristic maps.

The two main discoveries wrt. these predicates as characteristic maps are:

(1) in general, these predicates $X \rightarrow 1+1$ do not form a Boolean algebra, as one might have expected, but an effect algebra - or more specifically, an effect module;

(2) these predicates $X \rightarrow 1+1$ look rather simple, but, interpreted in different categories, they naturally capture various descriptions in a common framework, including for instance ordinary set-theoretic predicates $X \rightarrow\{0,1\}$, fuzzy predicates $X \rightarrow[0,1]$, effects in a Hilbert space or $C^{*}$-algebra, idempotents in a ring, complementable elements in a distributive lattice, etc.

For instance, for a ring $R$ we show that predicates $R \rightarrow 1+1$, in the opposite of the category of rings, correspond to idempotents in $R$, and form an effect algebra. When the ring $R$ is commutative, this effect algebra is actually a Boolean algebra. The fact that idempotents in a commutative ring form a Boolean algebra is well-known (see [48]). But the fact that these idempotents form an effect algebra in the non-commutative case is new (as far as we know).

There are four categories that will be used as leading examples. We briefly mention them now, but refer to the Appendix for more details.

(1) The category Sets of sets and functions, as model of deterministic computation with Boolean logic.

(2) The Kleisli category $\mathcal{K} \ell(\mathcal{D})$ of the distribution monad $\mathcal{D}$ on the category Sets, as model for discrete probabilistic computation and quantitative (fuzzy) logic.

(3) The Kleisli category $\mathcal{K} \ell(\mathcal{G})$ of the Giry monad $\mathcal{G}$ on the category Meas of measurable spaces; this category $\mathcal{K} \ell(\mathcal{G})$ is used for continuous probabilistic computation and quantitative logic.

(4) The opposites $\left(\mathbf{C s t a r}_{\mathrm{PU}}\right)^{\mathrm{op}}$ and $\left(\mathbf{C s t a r}_{\mathrm{CPU}}\right)^{\mathrm{op}}$ of the categories of $C^{*}$-algebras and (completely) positive unital maps, as models for quantum computation and logic. The (common) subcategory of commutative $C^{*}$-algebras and positive unital maps captures the probabilistic case.

In our axiomatisation of key aspects of these (and a few other) categories we start from predicates as maps of the form $X \rightarrow 1+1$. We then presents four successive assumptions on a category that are important, so we think, for the right leg in Figure1. These assumptions on a category $\mathbf{B}$ can be summarised as follows.

(1) The category B is an 'effectus', that is, it has a final object 1 and finite coproducts $0,+$ satisfying some mild properties. Then we prove that the predicates $X \rightarrow 1+1$ form an effect module. These predicates are related, via an adjunction, to states, which are maps of the form $1 \rightarrow X$ in $\mathbf{B}$. Moreover, for these predicates $p$ and states $\omega$ we can define validity $\omega \models p$ via an abstract Born rule $p \circ \omega$, producing a scalar. This scalar may for instance live in $\{0,1\}$ or in $[0,1]$, corresponding to Boolean and probabilistic validity respectively. The table in Figure 2 gives an overview of the different forms that this uniform definition of validity $\models$ takes in different categories. The duality between states and effects is an intrinsic part of our framework. The situation can be summarised in a "state-and-effect" triangle as in Figure 3. Many more such triangles are described in 43 .

(2) For each $n$-test, consisting of $n$ predicates $p_{i}: X \rightarrow 1+1$ which add up to 1 , there is a (chosen) instrument map instr $\operatorname{in}_{p} X n \cdot X=X+\cdots+X$ in $\mathbf{B}$ that performs 


\begin{tabular}{|c|c|c|c|}
\hline category & predicate $X \stackrel{p}{\rightarrow} 1+1$ & state $1 \stackrel{\omega}{\rightarrow} X$ & validity $\omega \mid=p$ \\
\hline Sets & subset $p \subseteq X$ & element $\omega \in X$ & $\omega \in p$ \\
\hline $\mathcal{K} \ell(\mathcal{D})$ & fuzzy $p: X \rightarrow[0,1]$ & distribution $\omega \in \mathcal{D}(X)$ & $\sum_{x \in X} p(x) \cdot \omega(x)$ \\
\hline $\mathcal{K} \ell(\mathcal{G})$ & measurable $p: X \rightarrow[0,1]$ & $\begin{array}{l}\text { probability measure } \\
\omega \in \mathcal{G}(X)\end{array}$ & $\int p \mathrm{~d} \omega$ \\
\hline $\mathbf{D L}^{\mathrm{op}}$ & complementable $p \in X$ & prime filter $\omega \subseteq X$ & $p \in \omega$ \\
\hline $\mathbf{B A}^{\text {op }}$ & $p \in X$ & ultrafilter $\omega \subseteq X$ & $p \in \omega$ \\
\hline $\mathbf{R n g}^{\text {op }}$ & idempotent $p \in X$ & $\omega: X \rightarrow \mathbb{Z}$ & $\omega(p)$ \\
\hline \multirow{2}{*}{ 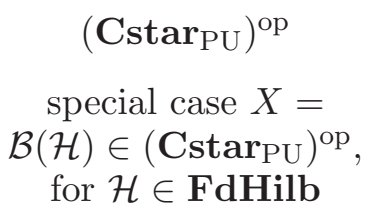 } & $p \in X$ with $0 \leq p \leq 1$ & $\omega: X \rightarrow \mathbb{C}$ & $\omega(p)$ \\
\hline & $\begin{array}{c}\mathcal{H} \stackrel{p}{\rightarrow} \mathcal{H} \\
\text { with } 0 \leq p \leq \mathrm{id}\end{array}$ & $\begin{array}{c}\text { density matrix } \\
\omega \in \mathcal{D} \mathcal{M}(\mathcal{H})\end{array}$ & $\operatorname{tr}(\omega p)$ \\
\hline
\end{tabular}

Figure 2: Examples of predicates $p$, states $\omega$, and validity $(\omega \models p)=p \circ \omega$ in different categories, where DL, BA, Rng, and FdHilb are the categories of distributive lattices, Boolean algebras, rings, and of finite-dimensional Hilbert spaces respectively. Validity $\omega \models p$ is either Boolean, in $\{0,1\}$, or probabilistic, in $[0,1]$, depending on what the scalars $1 \rightarrow 1+1$ are in the category at hand.

measurement. These maps are a categorical formalisation of (discrete) instruments in quantum theory, see [20, 66, 35. Intuitively, an instrument map sends an element in $X$ to a suitable spread over the $n$ coproduct options in the output $X+\cdots+X$, determined by the predicates $p_{i}$. Such a map instr $p$ may have a side-effect (aka. observer effect) on the system under observation, describing the state change resulting form measurement/observation. Such state changes are typical for the quantum world. We show that these side-effects do not occur in set-theoretic or probabilistic models, including commutative $C^{*}$-algebras. However, side-effects do arise in proper quantum models, given by non-commutative $C^{*}$-algebras.

Via these instruments instr $p$ a guarded command for a programming language and test operators for a dynamic logic can be defined. There are two of these test operations, labelled as 'test-andthen' and as 'test-then'. Both of them may have side-effects (in quantum models). Thus, side-effects have a prominent role in our framework.

(3) The category $\mathbf{B}$ has tensors $\otimes$ which interact appropriately with the previous two points. In particular, the unit of this tensor is the final object 1, giving a tensor with projections $X \leftarrow X \otimes Y \rightarrow Y$. These projections are used for weakening on predicates and for marginal / partial trace computations on states. They can be used to pinpoint dependence in probabilistic and entanglement in quantum models.

(4) Finally, an object $Q$ is assumed together with two states and an appropriate predicate that capture quantum bits (qubits). This is rather standard, but is required to model quantum computations. 


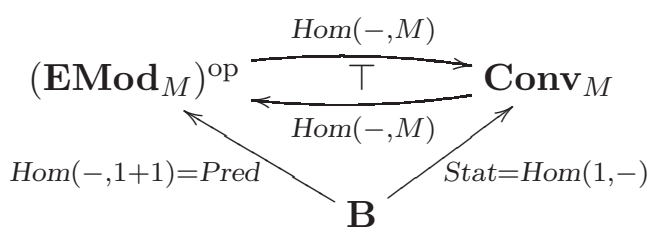

Figure 3: State-and-effect triangle for an effectus $\mathbf{B}$, with predicate and state functors, and with scalars $M=\operatorname{Pred}(1)=\operatorname{Stat}(1+1)=\operatorname{Hom}(1,1+1)$ in $\mathbf{B}$.

The first of these three points apply to set-theoretic, probabilistic, and to quantum models. Only the last, fourth point holds exclusively for quantum models (esp. $C^{*}$-algebras).

Thus, one of the achievements of this paper is the uniform description of these settheoretic, probabilistic and quantum models. Quantum mechanics is often described in terms of Hilbert spaces, see textbooks like [35, but also [2, 3]. However, it is the 'algebraic' formulation of quantum mechanics in term of $C^{*}$-algebras that fits in the current uniform framework. This algebraic approach stems from Heisenberg's matrix mechanics picture, see e.g. [58, 77].

This switch to $C^{*}$-algebras was one of three steps that were crucial in the development of this work. We list these steps explicitly.

(1) Not to use Hilbert spaces but $C^{*}$-algebras as (categorical) model for quantum computation.

(2) Not to use $C^{*}$-algebras with standard *-homomorphisms (preserving multiplication, involution and unit), but with (completely) positive unital maps. The latter preserve the unit and positive elements, and, if they are completely positive, preserve positive elements under every extension.

(3) Not to use the standard direction of maps between $C^{*}$-algebra, but to work in the opposite category, and understand computations (maps) between $C^{*}$-algebras as predicate transformers, working backwards.

These three points come together in the simple but crucial observation: maps of the form $A \rightarrow 1+1$, i.e. predicates, in the opposite of the category of $C^{*}$-algebras with (completely) positive unital maps are in one-to-one correspondence with elements $a \in A$ satisfying $0 \leq$ $a \leq 1$; these elements are called effects, and form an effect module. Also, instruments for $C^{*}$-algebras are given by Lüders rule, see (7.5), and form a completely positive map $A \rightarrow A+\cdots+A$, again in the opposite category.

From duality theory (see [48]) it is already known that some categories occur most naturally in dual form. A well-known example is the category cHA of complete Heyting algebras (aka. frames), whose opposite category $\mathbf{c H A}^{\mathrm{op}}$ even has a separate name, namely the category Loc of locales. Similarly, categories of $C^{*}$-algebras occur naturally in opposite form, as categories of non-commutative geometries. This is in line with Heisenberg's picture where computations act in opposite direction. Here we describe this phenomenon more generally as: maps between 'algebraic models of logics' can be understood as predicate transformers acting in the opposite direction. This applies for instance to categories of Boolean algebras, distributive lattices, or rings (like in Figure 2).

This paper is organised as follows. Background information about effect algebras and modules is provided in Section 2, and about our running probabilistic and quantum example categories in the Appendix. Predicates of the form $X \rightarrow 1+1$ in various categories are 
described in Section [3. Their effect structure is investigated subsequently in Section 4. States, as maps $1 \rightarrow X$, are added to the picture in Section 5. At that stage we can give our semantic framework involving the satisfaction relation $=$ (see the overview in Figure 2), predicate and state transformers, and the state-and-effect triangle in Figure 3 , Section 6 explains a side-topic, namely how predicates $X+Y \rightarrow 1+1$ on coproducts $X+Y$ correspond (via an isomorphism of effect modules) to pairs of predicates on $X$ and on $Y$. This general result includes familiar isomorphisms, like $\mathcal{P}(X+Y) \cong \mathcal{P}(X) \times \mathcal{P}(Y)$.

Section 7 introduces the next major contribution, namely a categorical formalisation of the notion of measurement instrument. Via these instruments we express what it means when a predicate (or more generally, a test) has a side-effect. This is studied more systematically in Section 8, where side-effect-freeness is related to commutativity in $C^{*}$-algebras. This gives mathematical expression to the idea that observations may disturb, which is typical for the quantum setting, as modelled by non-commutative $C^{*}$-algebras. These instruments are used to perform the action in a dynamic logic, involving 'andthen' and 'then' test operators $\langle p ?\rangle(q)$ and $[p ?](q)$, for predicates $p, q$. These operators are defined in the general setting provided by our axiomatisation. Instantiations in our running examples give familiar logical constructions, like conjunction and implication, multiplication and Reichenbach implication, or the sequential operations from [31, 32]. These logical test operators $\langle p ?\rangle(q)$ and $[p ?](q)$ satisfy some of the standard properties from dynamic logic, but they represent a new approach in that they capture side-effects of the action $p$, via the associated instrument that is used in their definition.

In Sections 10 and 11 the third and fourth assumptions are discussed, involving tensor products $\otimes$ and a special object $Q$ for qubits. The tensor product $\otimes$ comes with projections, which lead to weakening as predicate transformer and to marginals as state transformer. The composition operation on scalars turns out to be commutative in the presence of tensors — via a more-or-less standard, Eckmann-Hilton style argument - and allow us to pinpoint dependence and entanglement. We conclude in Section 11 by elaborating the superdense coding example in our newly developed setting.

\section{Preliminaries on effect algebras, effect modules, and Convex sets}

This paper uses categorical language to organise its material. It assumes a basic level of familiarity with category theory including (co)product and monoidal structure, adjunctions, and monads (including Kleisli and Eilenberg-Moore categories), see e.g. 7, 60. Also, it uses examples involving the distribution and Giry monad $\mathcal{D}$ and $\mathcal{G}$, Hilbert spaces, and $C^{*}$-algebras. Background information about these examples is relegated to the appendix. It may be consulted on a call-by-need basis.

This preliminary section does describe the basics of effect algebras, effect modules, and convex sets. This material is less standard, and an essential part of the approach presented here.

In order to define an effect algebra, we need the notion of partial commutative monoid (PCM). Before reading the definition of PCM, think of the unit interval $[0,1]$ with addition + . This + is obviously only a partial operation, which is commutative and associative in a suitable sense. This will be formalised next.

Definition 2.1. A partial commutative monoid (PCM) consists of a set $M$ with a zero element $0 \in M$ and a partial binary operation $\emptyset: M \times M \rightarrow M$ satisfying the three 
requirements below. They involve the notation $x \perp y$ for: $x \oslash y$ is defined; in that case $x, y$ are called orthogonal.

(1) Commutativity: $x \perp y$ implies $y \perp x$ and $x \oslash y=y \oslash x$;

(2) Associativity: $y \perp z$ and $x \perp(y \otimes z)$ implies $x \perp y$ and $(x \otimes y) \perp z$ and also $x \otimes(y \otimes z)=$ $(x \otimes y) \otimes z$

(3) Zero: $0 \perp x$ and $0 \oslash x=x$.

Later on we shall also use 'joint orthogonality' of elements $x_{1}, \ldots, x_{n}$. This means that the sum $x_{1} \oslash \cdots \otimes x_{n}$ is defined. The associativity property means that we can write such expressions $x_{1} \oslash \cdots \otimes x_{n}$ without brackets.

The notion of effect algebra extends a PCM with an orthocomplement $(-)^{\perp}$. It is due to [25], and in slightly different form also to [18] and [29]. The reference [24] gives an overview.

Definition 2.2. An effect algebra is a $\operatorname{PCM}(E, 0, \otimes)$ with an orthocomplement, that is, with a (total) unary operation $(-)^{\perp}: E \rightarrow E$, such that:

- $x^{\perp} \in E$ is the unique element in $E$ with $x \oslash x^{\perp}=1$, where $1=0^{\perp}$;

- $x \perp 1 \Rightarrow x=0$.

For such an effect algebra one defines,

$$
x \leq y \Longleftrightarrow \exists z \cdot x \otimes z=y \quad \text { and } \quad x \ominus y=\left(x^{\perp} \oslash y\right)^{\perp}, \text { for } x \geq y .
$$

We shall shortly see, in Lemma 2.3 (31), that there is at most one element $z$ with $x \oslash z=y$.

A homomorphism $E \rightarrow D$ of effect algebras is given by a function $f: E \rightarrow D$ between the underlying sets satisfying $f(1)=1$, and if $x \perp x^{\prime}$ in $E$ then both $f(x) \perp f\left(x^{\prime}\right)$ in $D$ and $f\left(x \otimes x^{\prime}\right)=f(x) \otimes f\left(x^{\prime}\right)$. Effect algebras and their homomorphisms form a category, written as EA.

The standard example of an effect algebra is the unit interval. In [0,1] one has $r \perp s$ iff $r+s \leq 1$, and in that case $r \otimes s=r+s$. The orthocomplement is $r^{\perp}=1-r$, obviously with $r \perp(1-r)$ and $r \otimes(1-r)=1$. But the 2-element set $2=\{0,1\}$ is also an example of an effect algebra - it is in fact the initial one. Hence both Booleans and probabilities form instances of the notion of effect algebra.

When writing $x \otimes y$ we shall implicitly assume that $x \otimes y$ is defined, i.e. that $x, y$ are orthogonal: $x \perp y$. Similarly for $\oslash$. The notation $\oslash$ might suggest that this operation is idempotent, but this is not the case (and not intended): for instance, in the unit interval $[0,1]$ one has $\frac{1}{2} \otimes \frac{1}{2}=1 \neq \frac{1}{2}$. But more generally, $x \otimes x=x$ only holds for $x=0$, since $x \otimes x=x=x \otimes 0$ yields $x=0$ by cancellativity, see Lemma 2.3 (3) below.

Aside: an alternative axiomatisation of the unit interval exists in terms of MV-algebras [14, 63, in which the sum operation is forced to be total, via truncation: $\min (r+s, 1)$. The partiality of $\otimes$ in effect algebras may look strange at first, but turns out to be quite natura 2, occurring in many categories (see Proposition 4.4 below).

\footnotetext{
${ }^{2}$ Interestingly, in his An Investigation of the Laws of Thought from 1854 George Boole himself considered disjunction to be a partial operation. He writes, on p.66 in the original edition: "Now those laws have been determined from the study of instances, in all of which it has been a necessary condition, that the classes or things added together in thought should be mutually exclusive. The expression $x+y$ seems indeed uninterpretable, unless it be assumed that the things represented by $x$ and the things represented by $y$ are entirely separate; that they embrace no individuals in common."
} 
We shall see many examples of effect algebras later on. Here we just mention that each orthomodular lattice is an effect algebra, in which elements $x, y$ are orthogonal iff $x \leq y^{\perp}$ iff $y^{\perp} \leq x$, and also that each Boolean algebra is an effect algebra with $x \perp y$ iff $x \wedge y=0$.

We summarise some basic properties, without proof.

Lemma 2.3. In an effect algebra one has:

(1) Orthocomplementing is an involution: $x^{\perp \perp}=x$;

(2) Top and bottom are each others orthocomplements: $1^{\perp}=0$ and $0^{\perp}=1$;

(3) Cancellation holds: $x \otimes y=x \otimes z$ implies $y=z$;

(4) Positivity (or zerosumfreeness) holds: $x \otimes y=0$ implies $x=y=0$;

$(5) \leq$ is a partial order with 1 as top and 0 as bottom element;

(6) $x \leq y$ implies $y^{\perp} \leq x^{\perp}$;

(7) $x \otimes y$ is defined iff $x \perp y$ iff $y \leq x^{\perp}$ iff $x \leq y^{\perp}$;

(8) $x \leq y$ and $y \perp z$ implies $x \perp z$ and $x \oslash z \leq y \otimes z$;

(9) $x \oslash y=z$ iff $y=z \ominus x$.

Homomorphisms of effect algebras preserve the sums $\otimes$ that exist. This is like for a probability measure $\mu: \Sigma \rightarrow[0,1]$ satisfying $\mu(U \cup V)=\mu(U)+\mu(V)$ but only if the measurable subsets $U, V \in \Sigma$ are disjoint, i.e. if the sum $U \otimes V$ is defined in the Boolean algebra $\Sigma$ of measurable subsets. Such a $\mu$ is thus a map of effect algebras. We mention two properties.

Lemma 2.4. Let $f: E \rightarrow D$ be a homomorphism of effect algebras.

(1) Then:

$$
f\left(x^{\perp}\right)=f(x)^{\perp} \quad f(0)=0 \quad x \leq x^{\prime} \Longrightarrow f(x) \leq f\left(x^{\prime}\right) .
$$

(2) If E, D happen to be Boolean algebras, then $f$ is also a map of Boolean algebras: the inclusion functor $\boldsymbol{B} \boldsymbol{A} \hookrightarrow \boldsymbol{E} \boldsymbol{A}$ is full and faithful - where $\boldsymbol{B} \boldsymbol{A}$ is the category of Boolean algebras.

Proof. For the first point notice that $1=f(1)=f\left(x \otimes x^{\perp}\right)=f(x) \oslash f\left(x^{\perp}\right)$, so $f\left(x^{\perp}\right)=f(x)^{\perp}$ by uniqueness of orthocomplements. In particular, $f(0)=f\left(1^{\perp}\right)=f(1)^{\perp}=1^{\perp}=0$. Monotonicity is trivial. For the second point one uses that a join $x \vee y$ in a Boolean algebra can equivalently be expressed as a disjoint join $(x \wedge \neg y) \vee(x \wedge y) \vee(y \wedge \neg x)$. Therefor it is preserved by a map of effect algebras.

A test, or more precisely, an $n$-test in an effect algebra $E$ is given by $n$ elements $e_{1}, \ldots, e_{n} \in E$ with $e_{1} \oslash \cdots \emptyset e_{n}=1$. Such tests will be used as the basis for measurement instruments in Section 7 An $n$-test in $E$ can be identified with a map of effect algebras $\mathcal{P}(n) \rightarrow E$. If $E$ is the set of effects $\mathcal{E} f(\mathcal{H})$ in a Hilbert space $\mathcal{H}$ - consisting of the positive maps $\mathcal{H} \rightarrow \mathcal{H}$ below the identity - then an $n$-test in $\mathcal{E} f(\mathcal{H})$ is an observable on the discrete measure space $n$, in the sense of $[35]^{3}$.

Two extensions of the notion of effect algebra will be used, namely extension of an effect algebra $E$ with:

- a multiplication $x \cdot y$ of its elements $x, y \in E$; we call such a structure an effect monoid;

\footnotetext{
${ }^{3}$ An observable on an arbitrary measure space $(X, \Sigma)$ can be described as a map of $\sigma$-effect algebras $\Sigma \rightarrow \mathcal{E} f(\mathcal{H})$, where the $\sigma$ refers to the preservation of countable joins of pairwise orthogonal elements, see [35, Defn. 3.5].
} 
- a scalar multiplication $r \bullet x$, where $x \in E$ and $r$ is a scalar, belonging for instance to $[0,1]$, or more generally to a given effect monoid; such a structure will be called an effect module.

In an effect monoid we require that the multiplication operation $\cdot$ is associative, preserves $0, \otimes$ in each argument separately, and satisfies $1 \cdot x=x=x \cdot 1$. An effect monoid is called commutative if its multiplication is commutative. The unit interval $[0,1]$ is an example of a commutative effect monoid, with the usual multiplication of probabilities. Similarly, the Booleans $\{0,1\}$ form a commutative effect monoid with multiplication (conjunction).

Given an effect monoid $M$, an effect algebra $E$ is an effect module over $M$ if there is a scalar multiplication $\bullet: M \times E \rightarrow E$ which preserves $0, \otimes$ in each argument separately and additionally satisfies: $1 \bullet x=x$ and $r \bullet(s \bullet x)=(r \cdot s) \bullet x$. A map of effect modules is a map of effect algebras that commutes with scalar multiplication. We write $\mathbf{E M o d}_{M}$ for the resulting category. There is an obvious forgetful functor $\mathbf{E M o d}_{M} \rightarrow \mathbf{E A}$, comparable to the forgetful functor Vect $_{K} \rightarrow \mathbf{A b}$ of vector spaces over a field $K$ to Abelian groups.

Here is an example of an effect module - many more will be given below. The set $[0,1]^{X}$ contains functions from a set $X$ to $[0,1]$ that can be understood as fuzzy predicates. This set $[0,1]^{X}$ is an effect algebra, via pointwise operations, with $p \perp q$ if $p(x)+q(x) \leq 1$ for all $x \in X$. It is also an effect module over $[0,1]$ with scalar multiplication $r \bullet p \in[0,1]^{X}$, for $r \in[0,1]$, given by $(r \bullet p)(x)=r \cdot p(x)$. Thus, $[0,1]^{X} \in \operatorname{EMod}_{[0,1]}$, for each set $X$.

In a trivial manner each effect algebra $E$ is an effect module over $\{0,1\}$, with obvious scalar multiplication $\{0,1\} \times E \rightarrow E$. This is like: each Abelian group is a trivial module over the (initial) ring $\mathbb{Z}$ of integers.

Remark 2.5. The notions of effect monoid and module can be described more abstractly: an effect monoid is a monoid in the category of effect algebras, just like a semiring is a monoid in the category of commutative monoids. In 44 it is shown that the category EA of effect algebras is symmetric monoidal with the two-element (initial) effect algebra $2=\{0,1\}$ as tensor unit. Then one can consider, in a standard way, the categories $\operatorname{Mon}(\mathbf{E A})$ and CMon(EA) of (commutative) monoids $2 \rightarrow M \leftarrow M \otimes M$ in the category EA of effect algebras.

For an effect monoid $M \in \operatorname{Mon}(\mathbf{E A})$ the category $\mathbf{E M o d}_{M}$ of effect modules over $M$ is the the category $\operatorname{Act}_{M}(\mathbf{E A})$ of $M$-actions $M \otimes E \rightarrow E$, see [60, VII,§4]. This resembles the category $\operatorname{Mod}_{S}$ of modules over a semiring $S$ which may be described as the category $\operatorname{Act}_{S}(\mathbf{C M o n})$ of commutative monoids with $S$-scalar multiplication. Effect modules over $[0,1]$ have appeared under the name 'convex effect algebras', see [68]. Via suitable totalisations of the partial operation $\varnothing$ (see [44]) it is shown in [45, Prop. 9] that the category $\mathbf{E M o d}_{[0,1]}$ is equivalent to the category of order unit spaces: (real) partially ordered vector spaces with an order unit 1 satisfying: for each vector $v$ there is an $n \in \mathbb{N}$ with $-n \cdot 1 \leq v \leq n \cdot 1$.

The Appendix $A .1$ describes the distribution monad $\mathcal{D}:$ Sets $\rightarrow$ Sets where $\mathcal{D}(X)$ consists of formal sums $\sum_{i} r_{i}\left|x_{i}\right\rangle$ with $r_{i} \in[0,1]$ satisfying $\sum_{i} r_{i}=1$. In [39] it is shown that such a distribution monad can be defined more generally wrt. a (commutative) effect monoid $M$ in place of $[0,1]$. This works as follows.

$$
\mathcal{D}_{M}(X)=\left\{m_{1}\left|x_{1}\right\rangle+\cdots+m_{k}\left|x_{k}\right\rangle \mid x_{i} \in X, m_{i} \in M \text { with } \emptyset_{i} m_{i}=1 \in M\right\} .
$$


Implicitly in this formulation we assume that the finite sum $\otimes_{i} m_{i}$ exists. It can be shown that $\mathcal{D}_{M}$ is a monad on Sets, just like $\mathcal{D}=\mathcal{D}_{[0,1]}$ is a monad. For the trivial effect monoid $\{0,1\}$ the associated monad $\mathcal{D}_{\{0,1\}}$ is the identity functor.

In a next step we can form the category $\operatorname{Conv}_{M}=\mathcal{E} \mathcal{M}\left(\mathcal{D}_{M}\right)$ of Eilenberg-Moore algebras of this monad $\mathcal{D}_{M}$. Its objects are 'convex sets over $M$ ', that is, sets $X$ in which for each formal convex sum $\sum_{i} m_{i}\left|x_{i}\right\rangle \in \mathcal{D}_{M}(X)$ an element $\emptyset_{i} m_{i} x_{i} \in X$ exists. Such convex sets can also be described in terms of 'weighted sums' $x+{ }_{r} y$, interpreted as $r x+(1-r) y$, see e.g. [76, 78, 38]. Morphisms in $\mathcal{E M}\left(\mathcal{D}_{M}\right)$ are affine maps; they preserve such convex sums $\otimes_{i} m_{i} x_{i}$.

In [45, Prop. 5] (based on [38]) a (dual) adjunction between convex sets over $[0,1]$ and effect modules over $[0,1]$ is described. This adjunction exists in fact for an arbitrary effect monoid $M$ - instead of $[0,1]$ — by using $M$ as dualising object. It formalises the duality between effects and states in quantum foundations, and also the dual relations between Heisenberg's and Schrödinger's view on quantum computation.

Proposition 2.6. Let $M$ be an effect monoid. By "homming into $M$ " one obtains an adjunction:

$$
\left(\boldsymbol{E M o d}_{M}\right)^{o p} \underset{\operatorname{CMod}(-, M)}{\stackrel{\operatorname{Conv}(-, M)}{\longleftarrow}} \operatorname{Conv}_{M}=\mathcal{E M}\left(\mathcal{D}_{M}\right)
$$

Proof. Given a convex set $X \in \operatorname{Conv}_{M}$, the homset $\operatorname{Conv}(X, M)$ of affine maps is an effect module, with $f \perp g$ iff $\forall x \in X . f(x) \perp g(x)$ in $M$. In that case one defines $(f \otimes$ $g)(x)=f(x) \otimes g(x)$. It is easy to see that this is again an affine function. Similarly, the pointwise scalar product $(m \bullet f)(x)=m \cdot f(x)$ yields an affine function. This mapping $X \mapsto \operatorname{Conv}(X, M)$ gives a contravariant functor since for $h: X \rightarrow X^{\prime}$ in $\mathbf{C o n v}_{M}$ precomposition with $h$ yields a map $(-) \circ h: \operatorname{Conv}\left(X^{\prime}, M\right) \rightarrow \operatorname{Conv}(X, M)$ of effect modules.

In the other direction, for an effect module $E \in \operatorname{EMod}_{M}$, the $\operatorname{homset} \operatorname{EMod}(E, M)$ of effect module maps yields a convex set: for a formal convex sum $\sum_{j} m_{j}\left|f_{j}\right\rangle$, where $f_{j}: E \rightarrow M$ in $\operatorname{EMod}_{M}$ and $m_{j} \in M$, we can define an actual sum $f: E \rightarrow M$ by $f(y)=\bigotimes_{j} m_{j} \bullet f_{j}(y)$. This $f$ forms a map of effect modules. Again, functoriality is obtained via pre-composition.

The dual adjunction between $\mathbf{E M o d}_{M}$ and $\mathbf{C o n v}_{M}$ involves a bijective correspondence that is obtained by swapping arguments.

\section{Predichtes and tests}

This section introduces predicates as maps of the form $X \rightarrow 1+1$ in a category, and more generally, tests as maps $X \rightarrow n \cdot 1=1+\cdots+1$. At this stage our only aim is to illustrate what such predicates/tests are in the categories of interest. The algebraic structure of predicates will be investigated in the next section.

In topos theory predicates are described as maps of the form $X \rightarrow \Omega$, where $\Omega$ is a special object that carries Heyting algebraic structure. The crucial topos property is that such maps $X \rightarrow \Omega$ correspond to subobjects of $X$. Here we use $1+1$ instead of $\Omega$. This looks extremely simple, but we shall see that predicates $X \rightarrow 1+1$ include many interesting examples, such as fuzzy predicates $X \rightarrow[0,1]$ taking values in the unit interval $[0,1]$ of probabilities. In this section we elaborate the overview of predicates given in Figure 2 in the introduction. 
Let's be more explicit about notation: we write 0 for the initial object in a category, with unique map !: $0 \rightarrow X$ to each object $X$. The coproduct object $X+Y$ of two objects $X, Y$ comes with two coprojections $\kappa_{1}: X \rightarrow X+Y$ and $\kappa_{2}: Y \rightarrow X+Y$ which are universal: for each pair of maps $f: X \rightarrow Z$ and $g: Y \rightarrow Z$ there is a unique cotuple $[f, g]: X+Y \rightarrow Z$ with $[f, g] \circ \kappa_{1}=f$ and $[f, g] \circ \kappa_{2}=g$. For two maps $h: X \rightarrow A$ and $k: Y \rightarrow B$ one writes $h+k=\left[\kappa_{1} \circ h, \kappa_{2} \circ k\right]: X+Y \rightarrow A+B$. The codiagonal map $X+X \rightarrow X$ is written as $\nabla=[\mathrm{id}, \mathrm{id}]$. These coproducts, coprojections and cotuples generalise to $n$-ary form: $X_{1}+\cdots+X_{n}$. If all these objects are the same, we have a so-called copower, written as $n \cdot X=X+\cdots+X$.

For the products $X \times Y$ we use standard notation, with projections $X \stackrel{\pi_{1}}{\leftarrow} X \times Y \stackrel{\pi_{2}}{\rightarrow} Y$ and tuples $\langle f, g\rangle: Z \rightarrow X \times Y$, for maps $f: Z \rightarrow X, g: Z \rightarrow Y$. The empty product is a final object 1 , with unique map !: $X \rightarrow 1$ for each object $X$. We shall frequently use that products in a category $\mathbf{B}$ form coproducts in the opposite category $\mathbf{B}^{\mathrm{op}}$.

Definition 3.1. Let $\mathbf{B}$ be a category with coproducts and with a final object $1 \in \mathbf{B}$. Let $n \in \mathbb{N}$ be non-zero.

(1) An $n$-test on an object $X \in \mathbf{B}$ is map $p: X \rightarrow n \cdot 1=1+\cdots+1$.

(2) A 2-test on $X$ is also called a predicate on $X$. We write $\operatorname{Pred}(X)=\operatorname{Hom}(X, 1+1)$ for the homset of predicates on $X$. There are true and false predicates $1_{X}, 0_{X} \in \operatorname{Pred}(X)$, defined as:

$$
1_{X}=\left(X \stackrel{!_{X}}{\longrightarrow} 1 \stackrel{\kappa_{1}}{\longrightarrow} 1+1\right) \quad 0_{X}=\left(X \stackrel{!_{X}}{\longrightarrow} 1 \stackrel{\kappa_{2}}{\longrightarrow} 1+1\right)
$$

Each predicate $p \in \operatorname{Pred}(X)$ has an orthocomplement $p^{\perp} \in \operatorname{Pred}(X)$ defined by swapping the outcomes:

$$
p^{\perp}=\left(X \stackrel{p}{\longrightarrow} 1+1 \stackrel{\left[\kappa_{2}, \kappa_{1}\right]}{\cong} 1+1\right)
$$

The predicates $\operatorname{Pred}(1)$ on the final object $1 \in \mathbf{B}$ play a special role and will be called scalars (sometimes probabilities).

(3) Each map $f: Y \rightarrow X$ in B yields a "substitution" or "reindexing" function $\operatorname{Pred}(f)=$ $(-) \circ f: \operatorname{Pred}(X) \rightarrow \operatorname{Pred}(Y)$. In this way we get a functor Pred: $\mathbf{B}^{\text {op }} \rightarrow$ Sets.

Several different notations are in use for this map Pred $(f)$, namely $f^{*}$ in categorical logic, $w p(f)$ for 'weakest precondition' in programming logic'. We shall use all of these interchangeably, so Pred $(f)=f^{*}=w p(f)=(-) \circ f$.

We often drop the subscripts for the predicates $0=0_{X}$ and $1=1_{X}$ when they are clear from the context. Obviously, $1^{\perp}=0$ and $0^{\perp}=1$. Also, $p^{\perp \perp}=p$. Thus we have 'double negation' built into our logic, in line with the right leg in Figure 1 The substitution function $f^{*}$ clearly preserves truth, false and orthocomplements.

Examples 3.2. We describe some of the motivating examples.

(1) In the category Sets we identify the $n$-fold copower $n \cdot=1+\cdots+1$ with the $n$-element set, commonly also written as $n$. An $n$-test on a set $X$ is thus a function $p: X \rightarrow n$ giving a partition of $X$ in $n$ subsets $p^{-1}(i) \subseteq X$, for $i \in n$. A predicate, or 2-test, is a predicate described as characteristic function $X \rightarrow 2=\{0,1\}$. The probabilities $\operatorname{Pred}(1)$ are the Booleans $\{0,1\}$.

More generally, in a topos, the maps $X \rightarrow 1+1$ correspond to the Boolean predicates. The set of scalars Pred(1) need not be as trivial as $\{0,1\}$. For instance, in Sets $^{2}$ one has four maps $(1,1) \rightarrow(1,1)+(1,1)=(1+1,1+1)$, namely $\left(\kappa_{i}, \kappa_{j}\right)$ for $i, j \in\{1,2\}$. 
(2) Our next example involves the distribution monad $\mathcal{D}$ on Sets from Appendix A.1, which is used to model discrete probabilistic computations. The associated Kleisli category $\mathcal{K} \ell(\mathcal{D})$ captures these computations, via stochastic matrices, as maps $X \rightarrow Y$ in $\mathcal{K} \ell(\mathcal{D})$, corresponding to functions $X \rightarrow \mathcal{D}(Y)$. An $n$-test $p$ on a set $X \in \mathcal{K} \ell(\mathcal{D})$ is a function $p: X \rightarrow \mathcal{D}(n)$. It assigns to each element $x \in X$ an $n$-tuple $p(x): n \rightarrow[0,1]$ of probabilities $p(x)(i) \in[0,1]$ which add up to 1 , that is, $\sum_{i} p(x)(i)=1$. A predicate on $X$ can be identified with a fuzzy predicate $X \rightarrow[0,1]$. The scalars Pred(1) are in this case the usual probabilities in the unit interval $[0,1] \subseteq \mathbb{R}$. Thus we use the bijective correspondences:

$\frac{\text { Kleisli map } X \longrightarrow 2}{\overline{\text { function } X \longrightarrow \mathcal{D}(2)}} \cong[0,1]$

(3) For continuous probabilistic computation one uses the Giry monad $\mathcal{G}$ on the category Meas of measurable spaces, see Appendix A.2. In the category Meas, and also in the Kleisli category $\mathcal{K} \ell(\mathcal{G})$ of the Giry monad $\mathcal{G}$ on Meas, the object $n \cdot 1$ is the $n$ element set with the discrete $\sigma$-algebra $\mathcal{P}(n)$. Therefore, $\mathcal{G}(n) \cong \mathcal{D}(n)$. Hence an $n$-test on $X \in$ Meas is a measurable function $X \rightarrow \mathcal{G}(n)$. In particular, a predicate on $X \in$ Meas is simply a measurable function $X \rightarrow[0,1]$, or a $[0,1]$-valued random variable, as used for instance in [40]. As before we have the probabilities $[0,1]$ as scalars: $\operatorname{Pred}(1) \cong \mathcal{G}(2) \cong \mathcal{D}(2) \cong[0,1]$.

(4) Let Cstar $_{\mathrm{PU}}$ be the category of (complex unital) $C^{*}$-algebras, with positive unital maps as morphisms between them, see Appendix A.4 The algebra $\mathbb{C}$ of complex numbers is initial in $\operatorname{Cstar}_{\mathrm{PU}}$, since the only positive unital map $\mathbb{C} \rightarrow A$ to an arbitrary $C^{*}$ algebra $A$ is the map $z \mapsto z \cdot 1$, where $1 \in A$ is the unit. The category $\operatorname{Cstar}_{\mathrm{PU}}$ has binary products $A_{1} \oplus A_{2}$, given by the cartesian product of the underlying spaces, with coordinate-wise operations, and supremum norm $\left\|\left(a_{1}, a_{2}\right)\right\|=\left\|a_{1}\right\| \vee\left\|a_{2}\right\|$. The singleton space $\{0\}$ is the final object in Cstar $_{\mathrm{PU}}$.

We shall be working in the opposite category $\left(\text { Cstar }_{\mathrm{PU}}\right)^{\mathrm{op}}$. It thus has coproducts $\oplus$ and a final object $\mathbb{C}$. The $n$-fold coproduct $n \cdot 1$ is then the $n$-fold cartesian product $\mathbb{C}^{n}$. An $n$-test on $A \in$ Cstar $_{\mathrm{PU}}$ is a map $A \rightarrow \mathbb{C}^{n}$ in $\left(\text { Cstar }_{\mathrm{PU}}\right)^{\text {op }}$, that is, a positive unital map $p: \mathbb{C}^{n} \rightarrow A$. It can be identified with an $n$-tuple of effects $e_{i}=p(|i\rangle) \in[0,1]_{A}$ with $e_{1} \oslash \cdots \otimes e_{n}=1$. Indeed, $|i\rangle \in \mathbb{C}^{n}$ is positive, and so $e_{i}=p(|i\rangle) \geq 0$ in $A$. And $\bigotimes_{i} e_{i}=\sum_{i} p(|i\rangle)=p\left(\sum_{i}|i\rangle\right)=p(1)=1$. A predicate on $A$ can be identified with a pair of predicates $e_{1}, e_{2} \in[0,1]_{A}$ with $e_{1}+e_{2}=1$. Hence $e_{2}=1-e_{1}$, so we can identify the predicate with a single effect $e \in[0,1]_{A}$, where $e^{\perp}=1-e$. Thus we have the fundamental correspondences:

$\begin{array}{cl}\frac{A \longrightarrow 2}{\overline{\mathbb{C}^{2} \longrightarrow A}} & \text { in }\left(\mathbf{C s t a r}_{\mathrm{PU}}\right)^{\mathrm{op}} \\ \text { effect in }[0,1]_{A} \subseteq A & \end{array}$

(This correspondence also works for completely positive unital maps, since a positive map $f: A \rightarrow B$ is automatically completely positive if either $A$ or $B$ is commutative.)

The truth predicate in $[0,1]_{A}$ is obtained by applying the map ! $\circ \pi_{1}: \mathbb{C}^{2} \rightarrow \mathbb{C} \rightarrow A$ to $(1,0) \in \mathbb{C}^{2}$. It yields the top/unit element $1 \in[0,1]_{A}$. Similarly, false is obtained by applying ! $\circ \pi_{2}$ to the pair $(1,0)$; this yields the bottom/zero element $0 \in[0,1]_{A}$. 
The scalars for $C^{*}$-algebras are the predicates $\operatorname{Pred}(\mathbb{C})$ on the complex numbers, and correspond to the usual probabilities $[0,1]$.

For a map $f: B \rightarrow A$ in $\left(\text { Cstar }_{\mathrm{PU}}\right)^{\text {op }}$ the associated substitution function $f^{*}: \operatorname{Pred}(A) \rightarrow$ $\operatorname{Pred}(B)$ is defined by function application: an effect $e \in[0,1]_{A}$ is sent to the effect $f(e) \in[0,1]_{B}$.

We briefly describe what happens in the case we do not use positive unital (PU) but the more common *-homomorphisms between $C^{*}$-algebras, i.e. the multiplicativeinvolutive-unital (MIU) maps. It is easy to see that predicates $A \rightarrow 2$ in (Cstar $\left._{\text {MIU }}\right)^{\text {op }}$ correspond to projections in $A$, that is, to idempotent effects $a \in[0,1]_{A}$. The resulting scalars are the Booleans $\{0,1\}$. Hence in the MIU-case the logic is no longer quantitative (with $[0,1]$ as scalars). But the projections themselves do not form a Boolean algebra, in general.

(5) Let Rng be the category of rings (with unit), with ring homomorphisms between them. The set of integers $\mathbb{Z}$, with its standard ring structure, is initial in the category $\mathbf{R n g}$, and thus final in the opposite category $\mathbf{R n g}{ }^{\mathrm{op}}$. We shall write it simply as 1 . The coproduct $1+1 \in \mathbf{R n g}^{\text {op }}$ is then the cartesian product $\mathbb{Z} \times \mathbb{Z}$. We claim that there are bijective correspondences, for $R \in \mathbf{R n g}$,

$$
\begin{array}{cc}
\frac{R \longrightarrow 1+1}{\stackrel{\mathbb{Z}^{2}-f \rightarrow R}{\longrightarrow}} & \text { in } \mathbf{R n g}^{\text {op }} \\
\hline \hline \text { idempotents } e \in R &
\end{array}
$$

The correspondence arises as follows. Given a ring homomorphism $f: \mathbb{Z}^{2} \rightarrow R$ we get an element $e_{f}=f(1,0) \in R$ which is idempotent: $e_{f}^{2}=f(1,0)^{2}=f\left((1,0)^{2}\right)=f(1,0)=e_{f}$. In the other direction, given an idempotent $e \in R$ we define $f_{e}: \mathbb{Z}^{2} \rightarrow R$ by $f_{e}(n, m)=$ $n \cdot e+m \cdot(1-e)$. In order to prove that $f_{e}$ is a ring homomorphism, in particular that it preserves multiplication, one uses that $e$ is an idempotent.

It is an elementary fact that the idempotent elements in a commutative ring form a Boolean algebra, see e.g. [48, I, 1.9]. We shall see that in the non-commutative case these idempotents form an effect algebra, see Proposition 4.8. The scalars are the idempotents of the initial ring $\mathbb{Z}$, which are the elements $\{0,1\}$. An $n$-test in $\mathbf{R n g}^{\mathrm{op}}$, that is, a ring homomorphism $\mathbb{Z}^{n} \rightarrow R$ can be identified with an $n$-tuple $e_{1}, \ldots, e_{n}$ of idempotent elements of $R$ satisfying not only $e_{1}+\cdots+e_{n}=1$ but also $e_{i} \cdot e_{j}=0$ for $i \neq j$. The latter multiplication property follows from the fact that these maps $\mathbb{Z}^{n} \rightarrow R$ preserve multiplication.

(6) We write DL for the category of distributive lattices — with finite joins $0, \vee$ and meets $1, \wedge$ distributing over each other - and lattices homomorphisms preserving these finite joins and meets. The 2-element lattice $2=\{0,1\}$ is initial in DL. In the opposite category $\mathbf{D L}^{\text {op }}$ a predicate $L \rightarrow 1+1$ is thus a lattice homomorphism $f: 2 \times 2 \rightarrow L$. It can be identified with an element $x \in L$ that has a complement: there is an $x^{\prime} \in L$ with $x \wedge x^{\prime}=0$ and $x \vee x^{\prime}=1$. It is easy to see that such a complement, if it exists, is necessarily unique. These 'complementable' elements in $L$ form a Boolean sublattice of $L$. The scalars are the elements of the 2-element lattice $\{0,1\}$ - which both have a complement. An $n$-test is an $n$-tuple of elements $x_{1}, \ldots, x_{n}$ with $x_{1} \vee \cdots \vee x_{n}=1$ and $x_{i} \wedge x_{j}=0$ for $i \neq j$.

(7) We conclude with three examples, as 'sanity check'. In the category BA of Boolean algebras, the two element algebra $2=\{0,1\}$ is initial, and maps of Boolean algebras 
$2 \times 2 \rightarrow B$ correspond to elements of $B$. Hence all elements of $B$ occur as predicates $B \rightarrow$ $1+1$ in $\mathbf{B A}^{\mathrm{op}}$. Similarly, in the category EMod of effect modules over $[0,1]$, this unit interval $[0,1]$ is initial, and effect module maps $[0,1]^{2} \rightarrow E$ correspond to elements of $E$. In the category $\mathbf{C o n v}=\mathcal{E} \mathcal{M}(\mathcal{D})$ of convex sets, the coproduct $1+1$ is the unit interval $[0,1]$, since $1+1 \cong \mathcal{D}(1)+\mathcal{D}(1) \cong \mathcal{D}(1+1) \cong[0,1]$. Thus, the predicates $X \rightarrow 1+1$ in Conv are the affine maps $X \rightarrow[0,1]$, as used in the functor $\operatorname{Hom}(-,[0,1]):$ Conv $\rightarrow$ EMod $^{\text {op }}$ in the adjunction Conv $\rightleftarrows$ EMod $^{\mathrm{op}}$ in Proposition 2.6.

One may wonder why several of the categories in the above list occur in opposite form $(-)^{\text {op }}$. The appropriate way to see a morphism $L \rightarrow K$ between two "logical" structures $L, K$ as a computation is to interpret it as a predicate transformer computation going in the other direction, from $K$ to $L$ : it takes predicates on the 'post' state to predicates on the 'pre' state of the computation. For $C^{*}$-algebras this corresponds to Heisenberg's view on quantum computation. In Dijkstra's weakest precondition semantics [23] there is bijective correspondence between:

computations, as functions $X \stackrel{f}{\longrightarrow} \mathcal{P}(Y)$ in the Kleisli category of the powerset monad meet-preserving predicate transformers $\mathcal{P}(Y) \underset{{ }_{w p(f)}}{\longrightarrow} \mathcal{P}(X)$

Notice that these predicate transformers operate in the opposite direction. They can be described as a computations in the opposite category $\left(\mathbf{C L}_{\wedge}\right)^{\text {op }}$ of complete lattices and meet-preserving functions. This is precisely like in the above 'logical' categories of the form $(-)^{\text {op }}$, see also [43, 42].

In general the predicates Pred(1), i.e. the probabilities, have a monoid structure. Later on, in Proposition 10.2, it will be shown that this monoid is commutative in presence of a (distributive) monoidal structure, following [52. However, this fact will not be used immediately.

Lemma 3.3. In a category $\boldsymbol{B}$ with coproduct + and a final object 1 the collection Pred(1) of scalars, i.e. of predicates $1 \rightarrow 1+1$ on 1 , has a monoid structure, given by Kleisli composition:

$$
s \cdot r=s \odot r=\left(1 \stackrel{r}{\longrightarrow} 1+1 \stackrel{\left[s, \kappa_{2}\right]}{\longrightarrow} 1+1\right) .
$$

The truth predicate $1=\kappa_{1}$ is unit for this multiplication, and the falsum predicate $0=\kappa_{2}$ is zero element (meaning $0 \cdot s=0=s \cdot 0$ ).

Proof. Easy, since this multiplication is Kleisli composition.

On the Boolean scalars $\{0,1\}$ this multiplication is conjunction, but on the unit interval $[0,1]$ of scalars in the other examples it is multiplication.

\section{The effect algebra structure on predicates}

Having seen predicates and tests in the previous section we now give a more precise description of the categorical properties that we require. These requirements will be formulated below as our first out of four assumptions. A category satisfying the first assumption is now called an effectus, since [47]. We shall use this name here too.

After the introduction of this first assumption, this section shows two things:

- that these assumptions give us effect module structure on predicates; 
- that the example categories described in the previous section satisfy these assumptions. Subsequent Sections 5 and 6 describe more consequences of the following assumptions.

In a category $\mathbf{B}$ with coproducts + and a final object $1 \in \mathbf{B}$ the coproduct $X_{1}+X_{2}$ in B comes with 'partial projections' $\triangleright_{i}: X_{1}+X_{2} \rightarrow X_{i}$, described in $\mathbf{B}$ as:

$$
X_{1}+1 \stackrel{\triangleright_{1}=\mathrm{id}+!}{\longleftrightarrow} X_{1}+X_{2} \stackrel{\triangleright_{2}=\left[\kappa_{2} \circ !, \kappa_{1}\right]}{\longrightarrow} X_{2}+1
$$

These partial projections form maps $\triangleright_{i}: X_{1}+X_{2} \rightarrow X_{i}$ in the Kleisli category $\mathbf{B}_{+1}$ of the lift monad $(-)+1$ on $\mathbf{B}$, where they can be described more symmetrically as $\triangleright_{1}=[i d, 0]$ and $\triangleright_{2}=[0, i d]$. They are natural in this Kleisli category, since $\triangleright_{i} \odot\left(f_{1}+f_{2}\right)=f_{i} \odot \triangleright_{i}$, where the + on the left-hand-side is understood in $\mathbf{B}_{+1}$ - which, in $\mathbf{B}$, is given by $f_{1}+f_{2}=$ $\left[\left(\kappa_{1}+\mathrm{id}\right) \circ f_{1},\left(\kappa_{2}+\mathrm{id}\right) \circ f_{2}\right]$. Obviously, there are $n$-ary versions $\triangleright_{i}: X_{1}+\cdots+X_{n} \rightarrow X_{i}$ of these partial projections.

Assumption 1. We use a category $\mathbf{B}$ that is an effectus. This means that it has a final object 1 and finite coproducts $(+, 0)$ such that diagrams of the following form are pullbacks in $\mathbf{B}$.

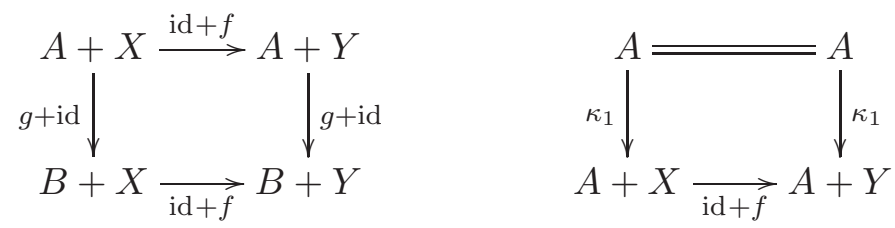

Additionally, we require that the following two maps are jointly monic in an effectus.

$$
(1+1)+1 \underset{\mathrm{X}=\left[\triangleright_{2}, \kappa_{2}\right]=\left[\left[\kappa_{2}, \kappa_{1}\right], \kappa_{2}\right]}{\stackrel{\mathrm{IV}=\left[\triangleright_{1}, \kappa_{2}\right]=\left[\left[\kappa_{1}, \kappa_{2}\right], \kappa_{2}\right]}{\longrightarrow}} 1+1
$$

The maps $\triangleright_{i}$ are the partial projections from (4.1). This joint monicity requirement means that if $f, g$ satisfy $\mathrm{I} \circ f=\mathrm{IV} \circ g$ and $\mathrm{X} \circ f=\mathrm{X} \circ g$, then $f=g$. The symbols $\mathrm{IV}$ and $\mathrm{X}$ should suggest what these two maps do.

The pullback requirements are rather mild and hold in many situations. The joint monicity of the maps (4.3) however is more restrictive, see also [39. Later on in this section, in Example 4.9, we show that it fails in the Kleisli category of the powerset monad. Thus, non-deterministic computation, in its standard form, does not fit in the current setting. Lemma 4.2 elaborates on this joint monicity requirement. At this stage we note that it says that in the Kleisli category of the lift monad the two partial projections $\triangleright_{1}, \triangleright_{2}: 1+1 \rightarrow 1$ are jointly monic.

At the end of Remark 6.4 we explain how the maps $\triangleright_{i}$ may be seen as partial projections in the Kleisli category of the lift monad, and how the joint monicity requirement translates to this Kleisli category.

First we obtain some more pullbacks. We show that assumptions about coproducts imply that they are disjoint: the coprojections are monic and have empty intersection (the pullback of different coprojections is empty). Hence our pullback requirements can be understood as a form of 'generalised' disjointness. In fact, the situation is:

$$
\left(\begin{array}{c}
\text { disjoint and stable } \\
\text { coproducts }
\end{array}\right) \Longrightarrow\left(\begin{array}{c}
\text { pullback requirements } \\
\text { in Assumption 1 } 14.2)
\end{array}\right) \Longrightarrow\left(\begin{array}{c}
\text { disjoint } \\
\text { coproducts }
\end{array}\right)
$$


Categories with coproducts that are disjoint and stable (under pullback) are also called extensive, see [13].

Lemma 4.1. In an effectus $\boldsymbol{B}$, the initial object $0 \in \boldsymbol{B}$ is strict, the coprojections $\kappa_{i}: A_{i} \rightarrow$ $A_{1}+A_{2}$ are monic and have empty intersection, and diagrams of the following form are pullbacks.
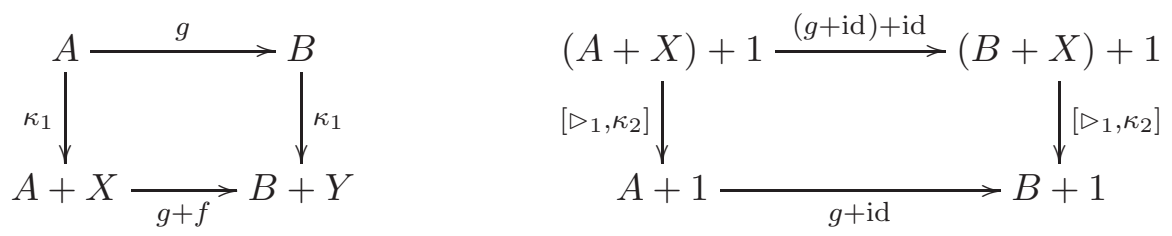

(The diagram on the right is a bit simpler when formulated in the Kleisli category $\boldsymbol{B}_{+1}$ of the lift monad, but we prefer to avoid confusion between $\boldsymbol{B}$ and $\boldsymbol{B}_{+1}$.)

Proof. Strictness of the initial object $0 \in \mathbf{B}$ means that each map $f: X \rightarrow 0$ is an isomorphism. For such a map, we have to prove that the composite $X \rightarrow 0 \rightarrow X$ is the identity. Consider the diagram:

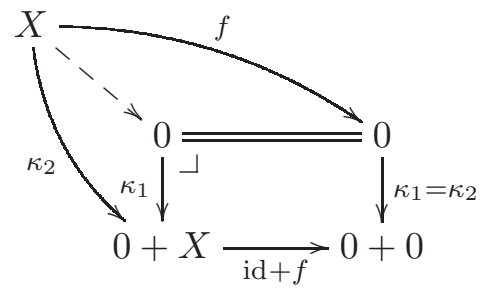

The rectangle is a pullback, as in (4.2). By initiality of 0 , we have $\kappa_{1}=\kappa_{2}: 0 \rightarrow 0+0$, so that the outer diagram commutes. Then we get the dashed map, as indicated, which must be $f: X \rightarrow 0$. But then $!_{X} \circ f=\left[!_{X}, \mathrm{id}_{X}\right] \circ \kappa_{1} \circ f=\left[!_{X}, \operatorname{id}_{X}\right] \circ \kappa_{2}=\mathrm{id}_{X}$.

We now prove a special case of the diagram on the left in (4.4) with $f=$ id:

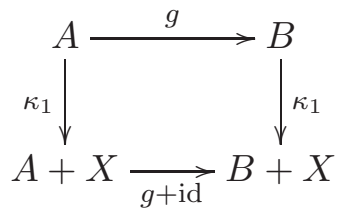

This is a pullback since it can be obtained from the square on the left in 4.2 ;

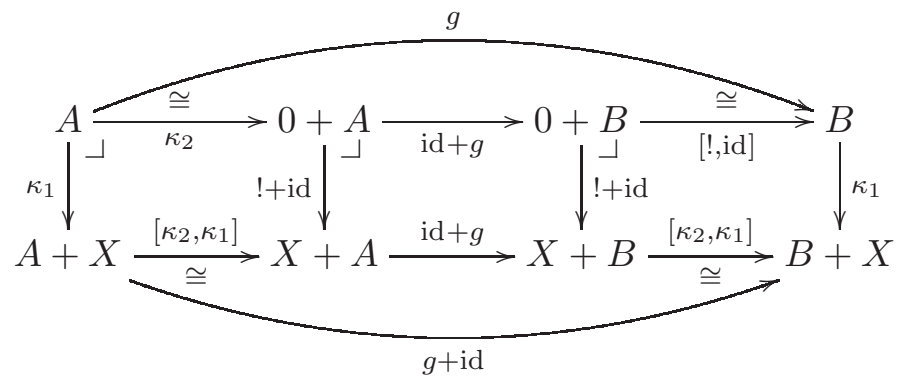


The square on the left in (4.4) is a pullback since it can be obtained from the square on the right in (4.2) and the previous square in (4.5):

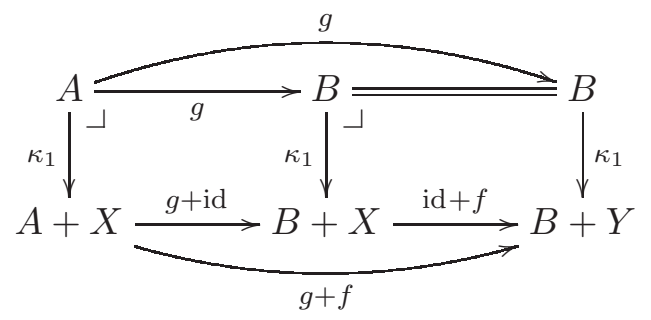

The first coprojection $\kappa_{1}: A \rightarrow A+X$ is monic since, using the pullback on the right in (4.2), we get a diagram as on the left below.
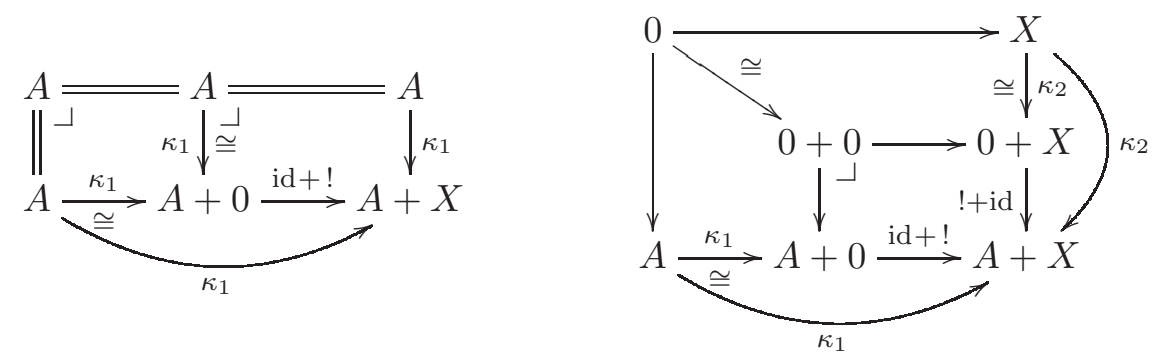

A similar argument applies to the second coprojection $\kappa_{2}$. The above diagram on the right shows that the intersection (pullback) of $\kappa_{1}, \kappa_{2}$ is the initial object 0 .

We turn to the square on the right in (4.4). Let $f: Y \rightarrow A+1$ and $h: Y \rightarrow(B+X)+1$ satisfy $(g+\mathrm{id}) \circ f=\left[\triangleright_{1}, \kappa_{2}\right] \circ h$. Consider the situation below, where in general, $\alpha$ is the associativity isomorphism $U+(V+W) \rightarrow(U+V)+W$, given explicitly by $\alpha=\left[\kappa_{1} \circ\right.$ $\left.\kappa_{1}, \kappa_{2}+\mathrm{id}\right]$ and $\alpha^{-1}=\left[\mathrm{id}+\kappa_{1}, \kappa_{2} \circ \kappa_{2}\right]$.

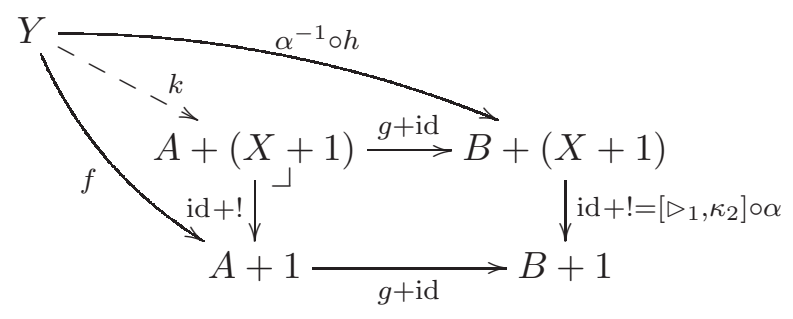

Then $k^{\prime}=\alpha \circ k: Z \rightarrow(X+A)+1$ is the required mediating map.

The joint monicity requirement in (4.3) says that the two maps $\mathrm{IV}, \mathrm{W}:(1+1)+1 \rightarrow 1+1$ are jointly monic. This simple formulation can be generalised in two ways: using arbitrary objects $X$ instead of 1 , and using arbitrary many 1's instead of only 2 . The equivalence of these generalisations to the formulation in (4.3) is due to Kenta Cho. (A slightly stronger formulation is used in [47.)

Lemma 4.2. Let $\boldsymbol{B}$ be a category with finite coproducts and a final object, in which the squares in (4.2) are pullbacks. Then the following statements are equivalent.

(1) The two maps $\mathrm{IV}, \mathrm{X}:(1+1)+1 \rightarrow 1+1$ in (4.3) are jointly monic.

(2) The two maps $\mathrm{IV}, \mathrm{W}:(X+X)+1 \rightarrow X+1$ are jointly monic, where as before, $\mathrm{IV}=$ $\left[\mathrm{id}+!, \kappa_{2}\right]$ and $\mathrm{X}=\left[\left[\kappa_{2} \circ !, \kappa_{1}\right], \kappa_{2}\right]$.

(3) For each $n \geq 1$, the $n$ maps $\left[\triangleright_{i}, \kappa_{2}\right]: n \cdot X+1 \rightarrow X+1$ are jointly monic. 
Proof. The implication (2) $\Rightarrow(3)$ works by induction. We concentrate on the implication (11) $\Rightarrow$ (2). Let $f, g: Y \rightarrow(X+X)+1$ satisfy $\left[\triangleright_{i}, \kappa_{2}\right] \circ f=\left[\triangleright_{i}, \kappa_{2}\right] \circ g: Y \rightarrow X+1$ for $i=1,2$. Consider the diagram:

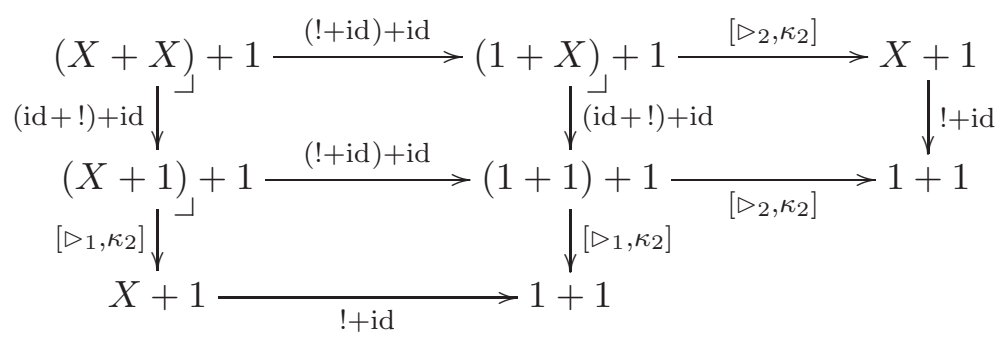

The lower and right rectangles are pullbacks, via the diagram on the right in (4.4). The upper left rectangle is a pullback by the diagram on the left in (4.2), using associativity of + .

The equations $\left[\triangleright_{i}, \kappa_{1}\right] \circ f=\left[\triangleright_{i}, \kappa_{1}\right] \circ g$ imply $\left[\triangleright_{i}, \kappa_{1}\right] \circ((!+!)+\mathrm{id}) \circ f=\left[\triangleright_{i}, \kappa_{1}\right] \circ$ $((!+!)+\mathrm{id}) \circ g$, so that $((!+!)+\mathrm{id}) \circ f=((!+!)+\mathrm{id}) \circ g$, because the maps $\left[\triangleright_{i}, \kappa_{1}\right]:(1+$ $1)+1 \rightarrow 1+1$ are jointly monic, by assumption. But then:

$$
\left\{\begin{aligned}
((!+\mathrm{id})+\mathrm{id}) \circ f=((!+\mathrm{id})+\mathrm{id}) \circ g & & \text { by the upper right pullback } \\
((\mathrm{id}+!)+\mathrm{id}) \circ f=((\mathrm{id}+!)+\mathrm{id}) \circ g & & \text { by the lower left pullback. }
\end{aligned}\right.
$$

Hence $f=g$ by the upper left pullback.

The main reason for the assumptions about coproducts in the beginning of this section lies in the following definition and result. The specific definition of sums $\emptyset$ via bounds that we use below goes back to [39]. But such techniques to get partially additive structure are much older, see [5].

Definition 4.3. Let $\mathbf{B}$ be a category satisfying Assumption 1 and let $p, q: X \rightarrow 1+1$ be two predicates on $X \in \mathbf{B}$.

(1) These two predicates are orthogonal, written as $p \perp q$, if there is a bound $b: X \rightarrow$ $(1+1)+1$ such that:

$$
\mathbb{I} \circ b=p \quad \text { and } \quad \mathbb{X} \circ b=q .
$$

According to Assumption 1 these two maps $\mathrm{IV}, \mathrm{W}:(1+1)+1 \rightarrow 1+1$ are jointly monic. Hence there is at most one such a bound $b$.

(2) If $p \perp q$ via bound $b$ then we define a new "sum" predicate $p \otimes q: X \rightarrow 1+1$ as:

$$
p \otimes q=(\nabla+\mathrm{id}) \circ b=([\mathrm{id}, \mathrm{id}]+\mathrm{id}) \circ b .
$$

Pictorially we have the following situation, with bound $b$ for predicates $p, q$.

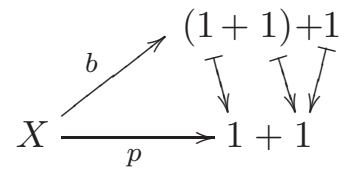

$p=I V \circ b$

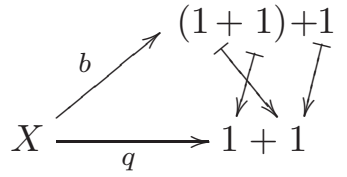

$1=X \circ b$

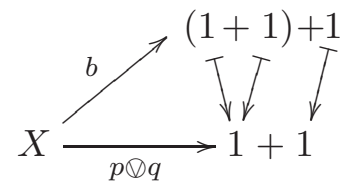

$p \otimes q=(\nabla+\mathrm{id}) \circ b$.

The next result shows how the pullback properties from Assumption 1 and Lemma 4.1 yield effect algebra structure on predicates. 
Proposition 4.4. Let $\boldsymbol{B}$ be a category satisfying Assumption 1 .

(1) For each object $X \in \boldsymbol{B}$ the collection Pred $(X)$ of predicates $X \rightarrow 1+1$ on $X$ forms an effect algebra, via $\otimes, 0,(-)^{\perp}$.

(2) For each map $f: Y \rightarrow X$ the substitution function $f^{*}=(-) \circ f: \operatorname{Pred}(X) \rightarrow \operatorname{Pred}(Y)$ is a map of effect algebras.

Thus we obtain a functor Pred: $\boldsymbol{B} \rightarrow \boldsymbol{E} \boldsymbol{A}^{o p}$.

Proof. The operation $\emptyset$ is commutative, since if $b$ is a bound for $p, q$, then $b^{\prime}=\left(\left[\kappa_{2}, \kappa_{1}\right]+\right.$ id) $\circ b$ is a bound for $q, p$ :

$$
\begin{aligned}
\mathbb{V} \circ b^{\prime} & =\left[\mathrm{id}, \kappa_{2}\right] \circ\left(\left[\kappa_{2}, \kappa_{1}\right]+\mathrm{id}\right) \circ b=\left[\left[\kappa_{2}, \kappa_{1}\right], \kappa_{2}\right] \circ b=q \\
\mathcal{W} \circ b^{\prime} & =\left[\left[\kappa_{2}, \kappa_{1}\right], \kappa_{2}\right] \circ\left(\left[\kappa_{2}, \kappa_{1}\right]+\mathrm{id}\right) \circ b \\
& =\left[\left[\kappa_{2}, \kappa_{1}\right] \circ\left[\kappa_{2}, \kappa_{1}\right], \kappa_{2}\right] \circ b=\left[\mathrm{id}, \kappa_{2}\right] \circ b=p .
\end{aligned}
$$

This $b$ and $b^{\prime}$ yield the same sum:

$$
\begin{aligned}
q \otimes p=(\nabla+\mathrm{id}) \circ b^{\prime} & =(\nabla+\mathrm{id}) \circ\left(\left[\kappa_{2}, \kappa_{1}\right]+\mathrm{id}\right) \circ b \\
& =\left(\left(\nabla \circ\left[\kappa_{2}, \kappa_{1}\right]\right)+\mathrm{id}\right) \circ b=(\nabla+\mathrm{id}) \circ b=p \otimes q .
\end{aligned}
$$

The zero predicate $0=\kappa_{2} \circ !_{X}: X \rightarrow 1+1$ is neutral element for $\emptyset$ : the equation $0 \otimes p=p$ holds via the bound $b=\left(\kappa_{2}+\mathrm{id}\right) \circ p: X \rightarrow(1+1)+1$.

For associativity of $\varnothing$ assume predicates $p, q, r: X \rightarrow 1+1$ where $p \perp q$, say via bound $a$, and $(p \otimes q) \perp r$, via bound $b$. Thus we have equations:

$$
\left\{\begin{array} { r l } 
{ W \circ a } & { = p } \\
{ \mathbb { N } \circ a } & { = q } \\
{ ( \nabla + \mathrm { id } ) \circ a } & { = p \otimes q }
\end{array} \quad \left\{\begin{array}{rl}
\mathrm{H} \circ b & =p \otimes q \\
\mathbb{N} \circ b & =r \\
(\nabla+\mathrm{id}) \circ b & =(p \otimes q) \otimes r .
\end{array}\right.\right.
$$

We consider the following pullback, occurring on the left in (4.2),

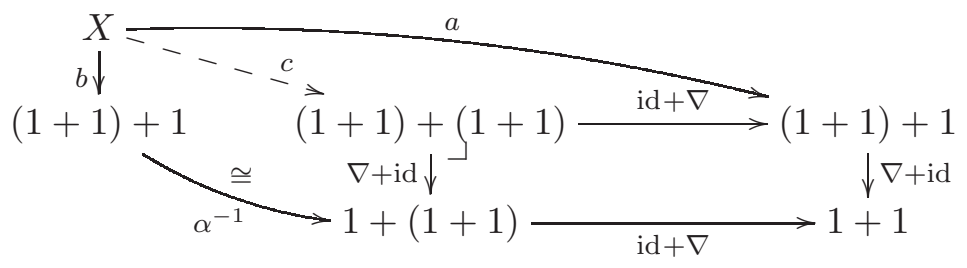

where $\alpha=\left[\kappa_{1} \circ \kappa_{1}, \kappa_{2}+\mathrm{id}\right]$ is the obvious associativity isomorphism.

We now take $c^{\prime}=\left[\left[\kappa_{2}, \kappa_{1} \circ \kappa_{1}\right], \kappa_{2}+\mathrm{id}\right] \circ c: X \rightarrow(1+1)+1$. This $c^{\prime}$ is a bound for $q$ and $r$, giving $q \perp r$. Next, the map $c^{\prime \prime}=\left[\kappa_{1}, \kappa_{2}+\mathrm{id}\right] \circ c: X \rightarrow(1+1)+1$ is a bound for $p$ and $q \otimes r=(\nabla+\mathrm{id}) \circ c^{\prime}$. Finally we obtain the associativity of $\emptyset$ :

$$
\begin{aligned}
p \otimes(q \otimes r)=(\nabla+\mathrm{id}) \circ c^{\prime \prime} & =(\nabla+\mathrm{id}) \circ\left[\kappa_{1}, \kappa_{2}+\mathrm{id}\right] \circ c \\
& =\left[\left[\kappa_{1}, \kappa_{1}\right],\left[\kappa_{1}, \kappa_{2}\right]\right] \circ c \\
& =\left[\left[\kappa_{1}, \kappa_{1}\right], \kappa_{2}\right] \circ\left[\kappa_{1} \circ \kappa_{1} \circ \nabla, \kappa_{2}+\mathrm{id}\right] \circ c \\
& =\left[\left[\kappa_{1}, \kappa_{1}\right], \kappa_{2}\right] \circ \alpha \circ(\nabla+\mathrm{id}) \circ c \\
& =(\nabla+\mathrm{id}) \circ b=(p \otimes q) \oslash r .
\end{aligned}
$$

It is not hard to see that $b=\kappa_{1} \circ p: X \rightarrow(1+1)+1$ is a bound for $p$ and $p^{\perp}=p \circ\left[\kappa_{2}, \kappa_{1}\right]$, yielding $p \otimes p^{\perp}=1$. 
Next we show that $p^{\perp}$ is the only predicate with $p \otimes p^{\perp}=1$. So assume also for $q: X \rightarrow 1+1$ we have $p \otimes q=1$, say via bound $b$. Then $\mathrm{I} \vee \circ b=p, \mathbb{X} \circ b=q$ and $(\nabla+\mathrm{id}) \circ b=1=\kappa_{1} \circ !_{X}$. We use the pullback (4.4) in:

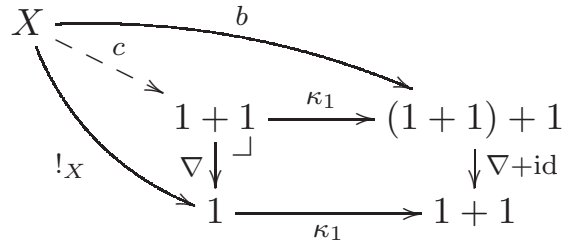

The fact that the bound $b$ is of the form $\kappa_{1} \circ c$ is enough to obtain $q=p^{\perp}$ :

$$
\begin{aligned}
p^{\perp}=\left[\kappa_{2}, \kappa_{1}\right] \circ p=\left[\kappa_{2}, \kappa_{1}\right] \circ\left[\mathrm{id}, \kappa_{2}\right] \circ b & =\left[\left[\kappa_{2}, \kappa_{1}\right], \kappa_{1}\right] \circ \kappa_{1} \circ c \\
& =\left[\left[\kappa_{2}, \kappa_{1}\right], \kappa_{2}\right] \circ \kappa_{1} \circ c \\
& =\left[\left[\kappa_{2}, \kappa_{1}\right], \kappa_{2}\right] \circ b=q .
\end{aligned}
$$

Finally, suppose $1 \perp p$; we must prove $p=0: X \rightarrow 1+1$. We may assume a bound $b$ with $\mathrm{I} \vee \circ b=1=\kappa_{1} \circ !_{X}$ and $\mathrm{X} \circ b=p$. Now we use the pullback on the right in (4.2) to obtain unique map $X \rightarrow 1$ as mediating map in:

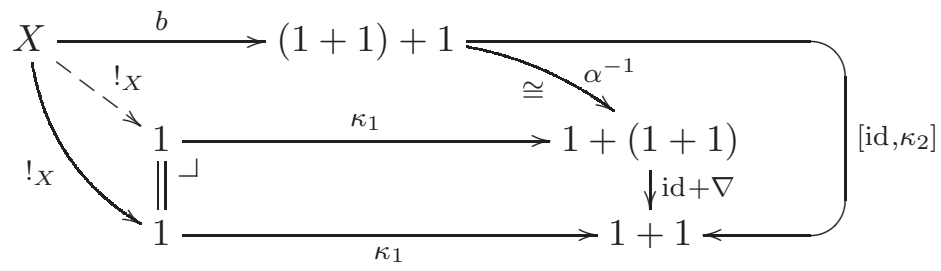

As a result, $b=\alpha \circ \kappa_{1} \circ !_{X}=\kappa_{1} \circ \kappa_{1} \circ !_{X}$. Then:

$$
p=\mathrm{X} \circ b=\left[\left[\kappa_{2}, \kappa_{1}\right], \kappa_{2}\right] \circ \kappa_{1} \circ \kappa_{1} \circ !_{X}=\kappa_{2} \circ !_{X}=0 .
$$

For the second point of the proposition we have to prove that substitution preserves $\emptyset$. So let $f: Y \rightarrow X$ be a map in $\mathbf{B}$, and let $p, q: X \rightarrow 1+1$ be orthogonal predicates, say via bound $b: X \rightarrow(1+1)+1$. Then $b \circ f$ is a bound for $p \circ f=f^{*}(p)$ and $q \circ f=f^{*}(q)$. Hence:

$$
f^{*}(p) \oslash f^{*}(q)=(\nabla+\mathrm{id}) \circ b \circ f=(p \otimes q) \circ f=f^{*}(p \otimes q) .
$$

In Lemma 3.3 we have seen that the collection Pred(1) of scalars (or probabilities) $1 \rightarrow 1+1$ carries a monoid structure $s \cdot r=\left[s, \kappa_{2}\right] \circ r$. By the previous result Pred(1) also carries an effect algebra structure. It turns out that these two structures interact appropriately to form an effect monoid, that is, a monoid in the monoidal category of effect algebras, see Remark 2.5 and [44].

Lemma 4.5. In a category $\boldsymbol{B}$ satisfying Assumption 1 the scalars Pred $(1)=\operatorname{Hom}(1,1+1)$ form an effect monoid. 
Proof. Let $s, r, r^{\prime}: 1 \rightarrow 1+1$ be three predicates on 1 , with $r \perp r^{\prime}$ via bound $b: 1 \rightarrow(1+1)+1$. We define the following two new bounds.

$$
c=\left(\begin{array}{c}
1 \\
\downarrow s \\
1+1 \\
\downarrow b+\mathrm{id} \\
\downarrow(1+1)+1 \\
(1+1)+1
\end{array}\right) \quad d=\left(\begin{array}{c}
1 \\
\downarrow b \\
(1+1)+1 \\
\downarrow(s+s)+\mathrm{id} \\
(1+1))+1 \\
\left.\left.\downarrow\left[\kappa_{2}\right]+\mathrm{id}, \kappa_{2}+\mathrm{id}\right], \kappa_{2}\right] \\
(1+1)+1 \\
(1+1)+1
\end{array}\right)
$$

The bound $c$ shows $r \cdot s \perp r^{\prime} \cdot s$ and $(r \cdot s) \oslash\left(r^{\prime} \cdot s\right)=\left(r \otimes r^{\prime}\right) \cdot s$. Similarly, $d$ proves $s \cdot r \perp s \cdot r^{\prime}$ and $(s \cdot r) \otimes\left(s \cdot r^{\prime}\right)=s \cdot\left(r \otimes r^{\prime}\right)$.

There is more structure: the effect monoid Pred(1) of scalars acts on every effect algebra $\operatorname{Pred}(X)$ of predicates in an appropriate manner, turning Pred $(X)$ into an effect module over Pred(1). Recall that we write $\mathbf{E M o d}_{M}$ for the category of effect modules over an effect monoid $M$. This subscript $M$ will be dropped when it is clear from the context.

For a scalar $s: 1 \rightarrow 1+1$ and a predicate $p: X \rightarrow 1+1$ we define the predicate $s \bullet p: X \rightarrow 1+1$ obtained by scalar multiplication as the following (Kleisli) composite.

$$
s \bullet p=\left(X \stackrel{p}{\longrightarrow} 1+1 \stackrel{\left[s, \kappa_{2}\right]}{\longrightarrow} 1+1\right) .
$$

Proposition 4.6. If $\boldsymbol{B}$ is a category satisfying Assumption 1 , then each collection of predicates Pred $(X)$ is an effect module over Pred(1), via the scalar multiplication $\bullet$ from (4.7). For $X=1$ this scalar multiplication $\bullet$ is the same as multiplication from Lemma 3.3.

The substitution functor $f^{*}=(-) \circ f: \operatorname{Pred}(X) \rightarrow \operatorname{Pred}(Y)$ induced by a map $f: Y \rightarrow$ $X$ in $\boldsymbol{B}$ preserves this effect module structure, in the sense that $f^{*}(s \bullet p)=s \bullet f^{*}(p)$. Thus, the functor Pred: $\boldsymbol{B} \rightarrow \boldsymbol{E} \boldsymbol{A}^{\text {op }}$ from Proposition 4.4 restricts to a functor Pred: $\boldsymbol{B} \rightarrow$ $\left(\boldsymbol{E M o d}_{\operatorname{Pred}(1)}\right)^{o p}$.

Proof. The scalar multiplication properties $1 \bullet p=p$ and $r \bullet(s \bullet p)=(r \cdot s) \bullet p$ follow from straightforward calculations. The fact that $\bullet$ is a bihomomorphism of effect algebras is shown as in the proof of Lemma 4.5. The rest is easy.

At this stage we like to emphasise that the relatively weak structure in Assumption 1 already gives us quite some logical structure, namely the indexed category of effect modules from the previous result. Our next aim is to investigate our assumptions more concretely in the examples listed in the previous section.

Example 4.7. The category of Sets is an effectus. In fact, every extensive category, with disjoint and universal coproducts [13, satisfies Assumption 1. Every topos is extensive, see e.g [9, Vol.3, §§5.9]). The proofs involve some elementary diagrammatic reasoning and will be skipped here. A predicate of the form $X \rightarrow 1+1=2$ in Sets is a characteristic function of a subset of $X$. For such predicates $P, Q \subseteq X$ the effect algebra sum $P \otimes Q$ is defined if $P \cap Q=\emptyset$, and in that case equals the union: $P \otimes Q=P \cup Q$. The scalars in Sets are the Booleans $2=\{0,1\}$, and scalar multiplication is trivial: $0 \bullet P=\emptyset$ and $1 \bullet P=P$.

The Kleisli category $\mathcal{K} \ell(\mathcal{D})$ of the distribution monad is als an effectus. We leave the verification of the pullback properties (4.2) to the interested reader and show that the maps $\mathrm{I}, \mathrm{X}:(1+1)+1 \rightarrow 1+1$ in (4.3) are jointly monic in $\mathcal{K} \ell(\mathcal{D})$. So let $\varphi, \psi \in \mathcal{D}(3)$ 
be distributions with equalities $\mathcal{D}(\mathbb{I V})(\varphi)=\mathcal{D}(\mathbb{I V})(\psi)$ and $\mathcal{D}(\mathbb{X})(\varphi)=\mathcal{D}(\mathbb{X})(\psi)$ in $\mathcal{D}(2)$ see (A.1) for the functor $\mathcal{D}$ on maps. In order to disambiguate matters we write $3=\{a, b, c\}$ and $2=\{u, v\}$. The functor applications yield convex sums:

$$
\mathcal{D}(\mathrm{IV})(\varphi)=\varphi(a)|u\rangle+(\varphi(b)+\varphi(c))|v\rangle \quad \text { and } \quad \mathcal{D}(\mathbb{W})(\varphi)=\varphi(b)|u\rangle+(\varphi(a)+\varphi(c))|v\rangle .
$$

Similarly for $\psi$. Hence the equations $\mathcal{D}(\mathbb{I V})(\varphi)=\mathcal{D}(\mathrm{IV})(\psi)$ and $\mathcal{D}(\mathbb{X})(\varphi)=\mathcal{D}(\mathbb{X})(\psi)$ immediately give us $\varphi(a)=\psi(a)$ and $\varphi(b)=\psi(b)$. We still need to prove $\varphi(c)=\psi(c)$. But $\varphi$ and $\psi$ are distributions, so their probabilities add up to one:

$$
\varphi(c)=1-\varphi(a)-\varphi(b)=1-\psi(a)-\psi(b)=\psi(c) .
$$

The sum $\emptyset$ of fuzzy predicates $p, q: X \rightarrow 1+1$ is defined if $p(x)+q(x) \leq 1$ for all $x \in X$. In that case one takes the pointwise sum $(p \otimes q)(x)=p(x)+q(x)$. Multiplication with a scalar $r \in[0,1]$ is also defined pointwise: $(r \bullet p)(x)=r \cdot p(x)$. Substitution $f^{*}(p)$, for $f: Y \rightarrow X$ in $\mathcal{K} \ell(\mathcal{D})$, is given by Kleisli composition $\odot$, and yields:

$$
f^{*}(p)(y)=(p \odot f)(y)=\sum_{x} p(x) \cdot f(y)(x) .
$$

Also the Kleisli category $\mathcal{K} \ell(\mathcal{G})$ of the Giry monad $\mathcal{G}$ is an effectus. The verification of Assumption 11 involve some properties of integrals - like splitting of an integral over a coproducts into a sum of integrals, see [40, Lemma 12] — that are skipped here. Since $\mathcal{G}(2) \cong \mathcal{D}(2) \cong[0,1]$ we obtain that predicates $X \rightarrow 1+1=2$ in $\mathcal{K} \ell(\mathcal{G})$ correspond to measurable functions $X \rightarrow[0,1]$. In particular, the scalars are the probabilities $[0,1]$. These measurable fuzzy predicates obviously carry the structure of an effect module over $[0,1]$, via pointwise operations. For a Kleisli map $f: Y \rightarrow \mathcal{G}(X)$, substitution $f^{*}(p)$ yields a predicate $Y \rightarrow[0,1]$ on $Y$ defined via Kleisli composition (written as $\odot$ ) as integration (see Section A.2):

$$
f^{*}(p)(y)=(p \odot f)(y)=p_{*}(f(y))=\int p \mathrm{~d} f(y) .
$$

This forms a 'continuous' version of the 'discrete' formula (4.8).

Next we look at opposite categories $\left(\mathbf{C s t a r}_{\mathrm{PU}}\right)^{\mathrm{op}}, \mathbf{R} \mathbf{n g}{ }^{\mathrm{op}}$ and $\mathbf{D L}^{\mathrm{op}}$ of $C^{*}$-algebras, rings, and distributive lattices. We shall start reasoning in $\mathbf{R n g}$, since the arguments carry over to the other categories in a straightforward manner.

We wish to prove that the squares of (4.4) are pullbacks in the category $\mathbf{R n g}^{\mathrm{op}}$. Thus we have to show that they are pushouts in $\mathbf{R n g}$, using products $\times$ instead of coproducts + :
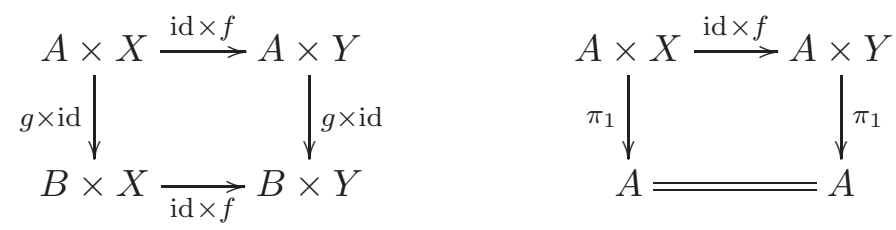

We start with the one on the left. Suppose we have ring homomorphisms $\alpha: A \times Y \rightarrow C$ and $\beta: B \times X \rightarrow C$ with $\alpha \circ($ id $\times f)=\beta \circ(g \times$ id $)$. Explicitly, this means that:

$$
\alpha(a, f(x))=\beta(g(a), x), \quad \text { for all } a \in A, x \in X .
$$

Then we define $\gamma: B \times Y \rightarrow C$ by:

$$
\gamma(b, y)=\alpha(0, y)+\beta(b, 0) .
$$


It is easy to see that $\gamma$ preserves addition. It preserves the unit, since:

$$
\begin{aligned}
\gamma(1,1)=\alpha(0,1)+\beta(1,0) & =\alpha(0, f(1))+\beta(1,0) \\
& =\beta(g(0), 1)+\beta(1,0)=\beta(0,1)+\beta(1,0)=\beta(1,1)=1 .
\end{aligned}
$$

Via a similar trick one shows that $\gamma$ preserves multiplication: the 'cross terms' disappear since:

$$
\alpha(0, y) \cdot \beta(b, 0)=\alpha(0, y) \cdot \alpha(0, f(1)) \cdot \beta(b, 0)=\alpha(0, y) \cdot \beta(g(0), 1) \cdot \beta(b, 0)=0 .
$$

Clearly, this $\gamma$ is the unique mediating map.

We turn to the above square on the right: given $\alpha: A \rightarrow B$ and $\beta: A \times Y \rightarrow B$ with $\alpha \circ \pi_{1}=\beta \circ($ id $\times f)$. The only possible mediating map is $\alpha: A \rightarrow B$. Hence we need to prove $\beta=\alpha \circ \pi_{1}: A \times Y \rightarrow B$, that is, $\beta(a, y)=\alpha(a)$, for all $a \in A, y \in Y$. Consider the function $\beta^{\prime}: Y \rightarrow B$ defined by $\beta^{\prime}(y)=\beta(0, y)$. This $\beta^{\prime}$ preserves finite sums $(+, 0)$ and multiplication $\cdot$, and satisfies:

$$
\beta^{\prime}(1)=\beta(0,1)=\beta(0, f(1))=\alpha(0)=0 .
$$

Then $\beta^{\prime}=0$, since $\beta^{\prime}(y)=\beta^{\prime}(1 \cdot y)=\beta^{\prime}(1) \cdot \beta^{\prime}(y)=0 \cdot \beta^{\prime}(y)=0$. But then we are done since:

$$
\beta(a, y)=\beta(a, 0)+\beta(0, y)=\beta(a, f(0))+\beta^{\prime}(y)=\alpha(a)+0=\alpha(a) .
$$

Next we investigate the jointly monic requirement from (4.3) in the category $\mathbf{R n g}^{\text {op }}$. When we translate this back to $\mathbf{R n g}$ we have to prove the corresponding maps $\mathrm{I}, \mathrm{W}: \mathbb{Z} \times \mathbb{Z} \rightarrow$ $\mathbb{Z} \times \mathbb{Z} \times \mathbb{Z}$ are jointly epic, where now $\mathbb{I V}=\left\langle\right.$ id, $\left.\pi_{2}\right\rangle$ and $\mathbb{X}=\left\langle\left\langle\pi_{2}, \pi_{1}\right\rangle, \pi_{2}\right\rangle$. Explicitly:

$$
\boldsymbol{I V}(k, m)=(k, m, m) \quad \text { and } \quad \mathbb{X}(k, m)=(m, k, m) .
$$

Let $f, g: \mathbb{Z} \times \mathbb{Z} \times \mathbb{Z} \rightarrow A$ satisfy $f \circ \mathrm{IV}=g \circ \mathrm{IV}$ and $f \circ \mathbb{W}=g \circ \mathrm{W}$. We obtain $f=g$ from:

$$
\begin{aligned}
f(k, m, \ell) & =f(k, \ell, \ell)+f(0, m-\ell, 0) \\
& =(f \circ \mathrm{IV})(k, \ell)+(f \circ \mathrm{W})(m-\ell, 0) \\
& =(g \circ \mathrm{IV})(k, \ell)+(g \circ \mathrm{W})(m-\ell, 0) \\
& =g(k, m, \ell) .
\end{aligned}
$$

The structure on predicates in a ring will be described separately in Proposition 4.8 below.

This line of reasoning showing that the category $\mathbf{R n g}{ }^{\text {op }}$ is an effectus can be transferred directly to the opposite $\mathbf{D L}^{\mathrm{op}}$ of the category of distributive lattices, and also to the opposite $\left(\mathbf{C s t a r}_{\mathrm{PU}}\right)^{\mathrm{op}}$ of the category of $C^{*}$-algebras with positive unital maps. The only point, in the latter case, where some care is needed is in the demonstration that $\beta^{\prime}(1)=0$ implies $\beta^{\prime}=0$, where $\beta^{\prime}$ is a positive unital map as defined above. It is an elementary result in the theory of $C^{*}$-algebras that a linear positive map $f$ with $f(1)=0$ must be the zero map. (For a proof, use that the operator norm $\|f\|$ equals $\|f(1)\|$, so $\|f(a)\| \leq\|f(1)\| \cdot\|a\|=0$, for each element $a$.)

The effect algebra structure from Definition 4.3, specialised to the subset of effects $[0,1]_{A} \subseteq A$ of a $C^{*}$-algebra $A$, is the standard one, with $e \emptyset d$ defined if $e+d \leq 1$, and in that case equal to $e+d$. The resulting order is the usual one for $C^{*}$-algebras.

We now formulate the structure of predicates on rings. Recall from Example 3.2 (5) that predicates $R \rightarrow 1+1$ in the opposite $\mathbf{R n g}^{\text {op }}$ of the category of rings are in one-to-one correspondence with idempotents of the ring $R$. 
Proposition 4.8. The effect algebra of idempotents $\operatorname{Pred}(R)=\left\{e \in R \mid e^{2}=e\right\}$ in a ring $R$ can be described as follows. Two idempotents e, $d \in \operatorname{Pred}(R)$ are orthogonal (i.e. $e \perp d$ ) iff $e \cdot d=0=d \cdot e$. In that case their effect algebra sum $e \emptyset d \in \operatorname{Pred}(R)$ equals their sum $e \otimes d=e+d$ in $R$. One has $e^{\perp}=1-e$. The induced order relation is: $e \leq d$ iff $e \cdot d=e=d \cdot e$.

In the special case that the ring $R$ is commutative, the effect algebra Pred $(R)$ of idempotents is actually a Boolean algebra with $e \wedge d=e d$ and $e \vee d=e+d-e d$.

These mappings can be made functorial, and yield a diagram:

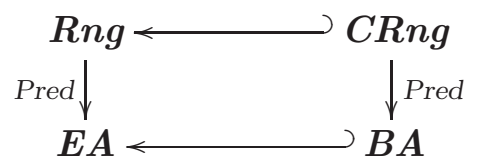

where $\boldsymbol{C R} \boldsymbol{n g}$ is the category of commutative rings.

The second part of this result - that the idempotents of a commutative ring form a Boolean algebra - is well-known, see for instance [48, V, Lemma 2.3]. The first part however - that in the general case the idempotents form an effect algebra - is less familiar, but the essence was already observed in [51].

Proof. For idempotent elements $e, d \in R$, corresponding to functions $f_{e}, f_{d}: \mathbb{Z}^{2} \rightarrow R$ as in Example 3.2 (5), we have $e \perp d$ iff there is a bound $b: \mathbb{Z}^{3} \rightarrow R$ with $b \circ\left\langle\right.$ id, $\left.\pi_{2}\right\rangle=f_{e}$ and $b \circ\left\langle\left\langle\pi_{2}, \pi_{1}\right\rangle, \pi_{2}\right\rangle=f_{d}$. This means that $b(n, m, m)=f_{e}(n, m)$ and $b(m, n, m)=f_{d}(n, m)$. Moreover, one obtains an idempotent $x=b(0,0,1) \in R$ satisfying:

$$
e+d+x=f_{e}(1,0)+f_{d}(1,0)+x=b(1,0,0)+b(0,1,0)+b(0,0,1)=b(1,1,1)=1 .
$$

But then $x=1-e-d$. Using that $b$ is a ring homomorphism we get:

$$
e \cdot d=b(1,0,0) \cdot b(0,1,0)=b((1,0,0) \cdot(0,1,0))=b(0,0,0)=0 .
$$

In the same way we can get $d \cdot e=0$.

In the reverse direction, if $e \cdot d=0=d \cdot e$, then we define a map $b: \mathbb{Z}^{3} \rightarrow R$ by:

$$
b(n, m, k)=n \cdot e+m \cdot d+k \cdot(1-e-d) .
$$

One immediately gets $b(n, m, m)=f_{e}(n, m)$ and $b(m, n, m)=f_{d}(n, m)$. With a bit more effort, using $e \cdot d=0=d \cdot e$, one proves that $b$ is a ring homomorphism. The resulting effect algebra sum $e \emptyset d \in R$ is given by their sum $e \emptyset d=e+d$ in $R$, corresponding to the function $f_{e \otimes d}: \mathbb{Z}^{2} \rightarrow R$ described by $f_{e \bowtie d}(n, m)=b(n, n, m)$, as in Definition 4.3.

Next we prove the characterisation of the order $\leq$ on predicates/idempotents. Assume $e \cdot d=e=d \cdot e$. We need to find an idempotent $x \in R$ with $e \cdot x=0=x \cdot e$ and $e+x=d$. Clearly, the only possible choice is $x=d-e$. This $x$ is idempotent and satisfies $e \perp x$ since

$$
e \cdot x=e \cdot(d-e)=e \cdot d-e^{2}=e-e=0=e-e=d \cdot e-e^{2}=(d-e) \cdot e=x \cdot e .
$$

In the other direction, assume $e \leq d$. Then there is an idempotent $x \in R$ with $e \perp x$ and $e+x=d$. The latter equation yields $x=d-e$. Orthogonality $e \perp x$ gives $e \cdot(e-d)=0=$ $(e-d) \cdot e$, so that $e \cdot d=e=d \cdot e$.

Recall from Example 3.2 (5) that for a ring $R$ an $n$-test $R \rightarrow n \cdot 1$ in $\mathbf{R n g}^{\text {op }}$ consists of a ring homomorphism $f: \mathbb{Z}^{n} \rightarrow R$, corresponding to $n$ idempotents $e_{i}=f(|i\rangle) \in R$ with $e_{1}+\cdots+e_{n}=1$ and $e_{i} \cdot e_{j}=0$ for $i \neq j$. Such an $n$-test is an essential ingredient of the Peirce decomposition $R \stackrel{\cong}{\longrightarrow} \bigoplus_{i, j} e_{i} R e_{j}$ of the ring $R$. 
Although the requirements in Assumption 1 look rather mild, they do not hold in all categories. Specifically, in the Kleisli category of the powerset monad they fail. This is significant, because this powerset monad is used for the semantics of non-deterministic computation. It is thus out of scope.

Remark 4.9. The Kleisli category $\mathcal{K} \ell(\mathcal{P})$ of the powerset monad $\mathcal{P}$ can be identified with the category of sets and relations between them. It does not satisfy the jointly monic requirement in Assumption 1. We construct a counterexample for $n=2$.

For a singleton set 1 we have $1+1+1 \cong\{a, b, c\}$ and $1+1 \cong\{u, v\}$. We can describe the two maps $\mathrm{IV}, \mathrm{X}: 3 \rightrightarrows 2$ in (4.3) as:

$$
\mathfrak{I V}(a)=u \quad \mathrm{IV}(b)=\mathrm{IV}(c)=v \quad \mathrm{X}(a)=\mathrm{X}(c)=v \quad \mathrm{X}(b)=u .
$$

The two subset $S=\{a, b\}$ and $T=\{a, b, c\}$ of 3 are clearly different but they satisfy:

$$
\mathcal{P}(\mathrm{IV})(S)=\{u, v\}=\mathcal{P}(\mathrm{I} V)(T) \quad \text { and } \quad \mathcal{P}(\mathrm{X})(S)=\{u, v\}=\mathcal{P}(\mathrm{X})(T) .
$$

Hence the two maps $\mathrm{J} V$ and $\mathrm{X}$ are not jointly monic in the category $\mathrm{Ke}(\mathcal{P})$.

On a different level, notice that the empty set 0 is a zero object in $\mathcal{K} \ell(\mathcal{P})$ : it is both initial and final. Hence $0+0 \cong 0$ is also a zero object, so that there is only one predicate $X \rightarrow 0+0$. The category $\mathcal{K} \ell(\mathcal{P})$ is not of interest in the current setting.

We finish this section with some observations about multiple sums $e_{1} \oslash \cdots \otimes e_{n}$.

Remark 4.10. Let B be an effectus i.e. a category for which Assumption 1 holds. Assume we have multiple predicates $p_{1}, \ldots, p_{n}: X \rightarrow 1+1$. We would like to express what it means that these $p_{i}$ are jointly orthogonal, i.e. that their sum $p_{1} \oslash \cdots \otimes p_{n}: X \rightarrow 1+1$ exists.

This can be done by using the $n$-ary maps $\left[\triangleright_{i}, \kappa_{2}\right]: n \cdot 1+1 \rightarrow 1+1$ from Assumption 1 , We say that $p_{1}, \ldots, p_{n}: X \rightarrow 1+1$ are pairwise orthogonal if there is a single bound $b: 1 \rightarrow(n+1) \cdot 1$ with $\left[\triangleright_{i}, \kappa_{2}\right] \circ b=p_{i}$, for each $i \in\{1, \ldots, n\}$. The sum is then defined as:

$$
p_{1} \oslash \cdots \otimes p_{n}=(X \stackrel{b}{\longrightarrow}(n+1) \cdot 1=n \cdot 1+1 \stackrel{\nabla+\mathrm{id}}{\longrightarrow} 1+1) .
$$

With these $n$-ary sums we can give an alternative description of $n$-tests, in line with how they are commonly understood, namely as predicates that add up to 1 . This generalises the bijective correspondences that we have in the context of $C^{*}$-algebras between:

$$
\frac{\frac{n \text {-tests } A \longrightarrow n \cdot 1 \text { in }\left(\mathbf{C s t a r}_{\mathrm{PU}}\right)^{\text {op }}}{\text { maps } \mathbb{C}^{n} \longrightarrow A \text { in } \text { Cstar }_{\mathrm{PU}}}}{\overline{\text { maps of effect modules }[0,1]^{n} \longrightarrow[0,1]_{A}}}
$$

Here is the general formulation.

Lemma 4.11. In a category satisfying Assumption 1 there is a bijective correspondence between:

$$
\frac{n \text {-tests } q: X \longrightarrow n \cdot 1}{\text { predicates } p_{1}, \ldots, p_{n}: X \longrightarrow 1+1 \text { with } p_{1} \oslash \cdots \otimes p_{n}=1}
$$


Proof. First suppose we have such a collection of predicates $p_{i}: X \rightarrow 1+1$ with $p_{1} \oslash \cdots \otimes p_{n}=$ 1. If $b: X \rightarrow(n+1) \cdot 1=n \cdot 1+1$ with $\left[\triangleright_{i}, \kappa_{2}\right] \circ b=p_{i}$ is the bound, like in Remark 4.10, then $(\nabla+\mathrm{id}) \circ b=\kappa_{1} \circ !_{X}$. We proceed like in diagram (4.6) in the proof of Proposition 4.4;

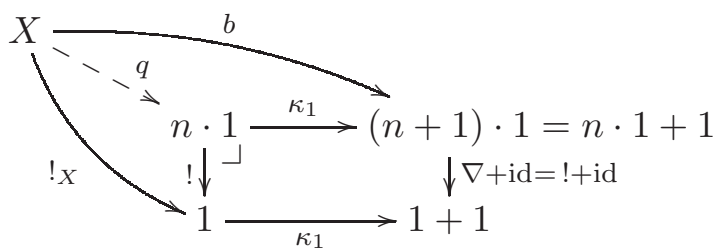

Thus we obtain an $n$-test $q: X \rightarrow n \cdot 1$. It satisfies:

$$
\left[\triangleright_{i}, \kappa_{2}\right] \circ \kappa_{1} \circ q=\left[\triangleright_{i}, \kappa_{2}\right] \circ b=p_{i} .
$$

This equation suggests how to proceed in the other direction: given an $n$-test $q: X \rightarrow$ $n \cdot 1$, define $p_{i}=\triangleright_{i} \circ q=\left[\triangleright_{i}, \kappa_{2}\right] \circ \kappa_{1} \circ q: X \rightarrow 1+1$. Then, by construction, $\kappa_{1} \circ q: X \rightarrow$ $(n+1) \cdot 1$ is a bound for these $p_{i}$, with:

$$
p_{1} \oslash \cdots \otimes p_{n}=(\nabla+\mathrm{id}) \circ \kappa_{1} \circ q=\kappa_{1} \circ \nabla \circ q=\kappa_{1} \circ !_{X}=1 .
$$

\section{States}

Having seen predicates as maps of the form $X \rightarrow 1+1$, we turn to states, and describe them as maps of the form $1 \rightarrow X$. They are sometimes called points. We define validity $\models$ for states and predicates, via an abstract Born rule, and show how these predicates and states give rise to a state-and-effect triangle.

Definition 5.1. In a category $\mathbf{B}$ with final object $1 \in \mathbf{B}$ we define a state to be a map of the form $1 \rightarrow X$. We write $\operatorname{Stat}(X)=\operatorname{Hom}(1, X)$ for the set of states.

For a map $f: X \rightarrow Y$ in $\mathbf{B}$ we get a function $\operatorname{Stat}(f)=f_{*}=f \circ(-): \operatorname{Stat}(X) \rightarrow$ Stat $(Y)$. This yields a functor Stat: $\mathbf{B} \rightarrow$ Sets.

In the category Sets, states of an object (set) $X$ are just elements of $X$. But in the Kleisli category $\mathcal{K} \ell(\mathcal{D})$, states $1 \rightarrow X$ correspond to functions $1 \rightarrow \mathcal{D}(X)$, and thus to distributions $\varphi \in \mathcal{D}(X)$, see Example 5.4 below. Similarly, states in the Kleisli category $\mathcal{K} \ell(\mathcal{G})$ of the Giry monad correspond to probability distributions $\phi \in \mathcal{G}(X)$. And states $1 \rightarrow A$ in the category $\left(\text { Cstar }_{\mathrm{PU}}\right)^{\mathrm{op}}$ are positive unital maps $A \rightarrow \mathbb{C}$, and thus states as they are commonly used in the theory of $C^{*}$-algebras.

Typically, states are closed under convex combinations. This can also be shown in the current context, where the scalars are of the form $1 \rightarrow 1+1$. It requires the generalised notion of convex set with respect to an effect monoid, as described towards the end of section 2 ,

Lemma 5.2. Let $\boldsymbol{B}$ be an effectus. For each object $X \in \boldsymbol{B}$ the set of states $\operatorname{Stat}(X)=$ $\operatorname{Hom}(1, X)$ is a convex set over the effect monoid $\operatorname{Pred}(1)=\operatorname{Hom}(1,1+1)$ of scalars in $\boldsymbol{B}$.

For each map $f: X \rightarrow Y$ in $\boldsymbol{B}$ the associated function $\operatorname{Stat}(f)=f_{*}=f \circ(-): \operatorname{Stat}(X) \rightarrow$ Stat $(Y)$ is affine. Thus, taking states yields a functor Stat: $\boldsymbol{B} \rightarrow \boldsymbol{C o n v}_{\text {Pred(1) }}$. 
Proof. Assume we have $n$ scalars $r_{1}, \ldots, r_{n}: 1 \rightarrow 1+1$ with $r_{1} \oslash \cdots \otimes r_{n}=1$ together with $n$ states $\omega_{1}, \ldots, \omega_{n}: 1 \rightarrow X$. The scalars correspond by (4.11) to an $n$-test $q: 1 \rightarrow n \cdot 1$. Then we define a convex sum of these states as:

$$
\emptyset_{i} r_{i} \omega_{i}=\left(1 \stackrel{q}{\longrightarrow} n \cdot 1 \stackrel{\left[\omega_{1}, \ldots, \omega_{n}\right]}{\longrightarrow} X\right) .
$$

More formally, we describe this definition as a function $\alpha: \mathcal{D}_{M}(\operatorname{Stat}(X)) \rightarrow \operatorname{Stat}(X)$, given by $\alpha\left(\sum_{i} r_{i}\left|\omega_{i}\right\rangle\right)=\bigotimes_{i} r_{i} \omega_{i}$. It forms an an Eilenberg-Moore algebra since:

$$
\begin{aligned}
(\alpha \circ \eta)(\omega) & =\alpha(1|\omega\rangle) \\
& =[\omega] \circ \mathrm{id} \\
& =\omega \\
\left.\left.(\alpha \circ \mu)\left(\sum_{i} r_{i}\left|\sum_{j \in J_{i}} s_{i j}\right| \omega_{i j}\right\rangle\right\rangle\right) & =\alpha\left(\sum_{i j} r_{i} \cdot s_{i j}\left|\omega_{i j}\right\rangle\right) \\
& =\emptyset_{i j}\left(r_{i} \cdot s_{i j}\right) \omega_{i j} \\
& =\bigotimes_{i} r_{i}\left(\emptyset_{j \in J_{i}} s_{i j} \omega_{i j}\right) \\
& \left.=\alpha\left(\sum_{i} r_{i} \mid \alpha\left(\sum_{j \in J_{i}} s_{i j}\left|\omega_{i j}\right\rangle\right)\right\rangle\right) \\
& \left.\left.=\left(\alpha \circ \mathcal{D}_{M}(\alpha)\right)\left(\sum_{i} r_{i}\left|\sum_{j \in J_{i}} s_{i j}\right| \omega_{i j}\right\rangle\right\rangle\right) .
\end{aligned}
$$

These convex sums make the set $\operatorname{Stat}(X)$ into a convex set. The sums are preserved by the functions $\operatorname{Stat}(f)$ since:

$$
\begin{aligned}
\operatorname{Stat}(f)\left(\bigotimes_{i} r_{i}\left|\omega_{i}\right\rangle\right) & =f \circ\left[\omega_{1}, \ldots, \omega_{n}\right] \circ q \\
& =\left[f \circ \omega_{1}, \ldots, f \circ \omega_{n}\right] \circ q=\emptyset_{i} r_{i}\left|\operatorname{Stat}(f)\left(\omega_{i}\right)\right\rangle .
\end{aligned}
$$

Next we define validity via composition. This definition is extremely simple but turns out to be very powerful since it can be interpreted in many categories and can mean many different things, depending on the meaning of composition, see the overview in Figure 2 ,

Definition 5.3. Given a state $\omega: 1 \rightarrow X$ on an object $X$ in an effectus, and a predicate $p: X \rightarrow 1+1$ on that same object, we can define the validity probability $\omega=p$ in Pred(1) as the scalar:

$$
\omega \models p=p \circ \omega: 1 \longrightarrow 1+1
$$

When the homset of scalars $\operatorname{Hom}(1,1+1)$ is $\{0,1\}$, this expression $\omega=p$ is a truth value. But $\omega \models p$ may also be a probability, when $[0,1]$ is set of scalars, as illustrated below. The definition (5.1) may be seen as Born's rule, in most elementary shape. It takes various forms, depending on the category in which $\models$ is interpreted. This will be elaborated next.

Examples 5.4. We shall see what the validity probability $\omega=p$ amounts to in our running examples. We encounter the formulations occurring in [21, Table 1], see also Figure 2, The idea of using integration as logical validity goes back to [55, 56].

We first consider the Kleisli category $\mathcal{K} \ell(\mathcal{D})$ of the distribution monad $\mathcal{D}$ on Sets. A state $\omega$ on a set $X \in \mathcal{K} \ell(\mathcal{D})$ is a function $1 \rightarrow \mathcal{D}(X)$, which corresponds to a discrete probability distribution $\omega \in \mathcal{D}(X)$. Thus $\operatorname{Stat}(X)=\mathcal{D}(X)$ and $f_{*}=\operatorname{Stat}(f)$ is the Kleisli extension map $\mathcal{D}(X) \rightarrow \mathcal{D}(Y)$, given by $f_{*}(\omega)(y)=\sum_{x} \omega(x) \cdot f(x)(y)$, for $f: X \rightarrow \mathcal{D}(Y)$. 
A predicate $p: X \rightarrow 1+1$ corresponds to function $p: X \rightarrow[0,1]$. The validity probability is obtained by Kleisli composition and looks as follows.

$$
\omega \models p=\sum_{x} \omega(x) \cdot p(x) \in[0,1] .
$$

In the Kleisli category $\mathcal{K} \ell(\mathcal{G})$ of the Giry monad this formula becomes integration: for a measurable space $X$, a state $\omega: 1 \rightarrow X$ in $\mathcal{K} \ell(\mathcal{G})$ is a probability measure $\omega \in \mathcal{G}(X)$. A predicate $p$ on $X$ is a measurable map $p: X \rightarrow[0,1]$. Then:

$$
\omega=p=\int p \mathrm{~d} \omega \in[0,1] .
$$

In the category $\mathbf{R n g}^{\text {op }}$ a state of a ring $R$ is a ring homomorphism $\omega: R \rightarrow \mathbb{Z}$. Such a map is sometimes called a $\mathbb{Z}$-point in algebraic geometry. A predicate is an idempotent $e \in R$, see Proposition 4.8. Validity becomes function application:

$$
\omega \models e=\omega(e) \in\{0,1\} .
$$

The outcome $\omega(e) \in \mathbb{Z}$ is in $\{0,1\}$ since $e$ is an idempotent, and $\omega$ is a ring homomorphism, so that $\omega(e)^{2}=\omega(e)$.

In the category $\mathbf{D L}^{\mathrm{op}}$ of distributive lattices a state of $L$ is a lattice homomorphism $\omega: L \rightarrow 2$. As is well-known, it may be identified with a prime filter $U=\omega^{-1}(1) \subseteq L$, that is, with a non-empty upset $U$ that is closed under meets and satisfies: $0 \notin U$ and $x \vee y \in U$ implies either $x \in U$ or $y \in U$. We can formulate validity $\models$ either in terms of states $\omega: L \rightarrow 2$ or in terms of prime filters $U \subseteq L$, as:

$$
\omega \models e=\omega(e) \in\{0,1\} \quad \text { or as } \quad U \models e=(e \in U) .
$$

where the predicate $e \in L$ has a complement, see Example 3.2 (6). Of course, instead of prime filters one may equivalently use prime ideals, corresponding to kernels $\omega^{-1}(0)$ of states $\omega$.

The same approach works in the opposite of the category of Boolean algebras, where a state of a Boolean algebra $B$ is a map of Boolean algebra $B \rightarrow 2$, which corresponds to an ultrafilter of $B$.

Next, we consider the opposite $\left(\mathbf{C s t a r}_{\mathrm{PU}}\right)^{\text {op }}$ of the category of $C^{*}$-algebras with positive unital maps. A state on a $C^{*}$-algebra $A$ is, as usual, a positive unital map $\omega: A \rightarrow \mathbb{C}$. It is a map $1 \rightarrow A$ in this opposite category $\left(\mathbf{C s t a r}_{\mathrm{PU}}\right)^{\mathrm{op}}$. For an effect/predicate $a \in[0,1]_{A}=$ $\{x \in A \mid 0 \leq x \leq 1\}$ we obtain the validity probability simply by function application:

$$
\omega=a=\omega(a) \in[0,1] .
$$

The outcome $\omega(a)$ is in the unit interval $[0,1] \subseteq \mathbb{R}$ because: $\omega(a) \geq 0$ since $a \geq 0$ and $\omega$ is positive, and $\omega(a) \leq 1$ since $a \leq 1$ and $\omega(1)=1$.

We briefly look at the special case where our $C^{*}$-algebra $A$ is the algebra $\mathcal{B}(\mathcal{H})$ of bounded linear maps on a finite-dimensional Hilbert space $\mathcal{H}$. We recall that the subset $\mathcal{D M}(\mathcal{H}) \hookrightarrow \mathcal{B}(\mathcal{H})$ of density matrices contains the positive operators with trace equal to one. Each such density matrix $\rho \in \mathcal{D} \mathcal{M}(\mathcal{H})$ gives rise to a state $\omega_{\rho}: \mathcal{B}(H) \rightarrow \mathbb{C}$, namely $\omega_{\rho}(f)=\operatorname{tr}(f \rho)$ where $\operatorname{tr}$ is the trace operation. Then, for an effect $E \in \mathcal{E} f(\mathcal{H})=[0,1]_{\mathcal{B}(\mathcal{H})}=$ $\{A: \mathcal{H} \rightarrow \mathcal{H} \mid 0 \leq A \leq \mathrm{id}\}$ on $\mathcal{H}$ we have the usual probability formula (see e.g [21]):

$$
\left(\omega_{\rho} \models E\right)=\omega_{\rho}(E)=\operatorname{tr}(E \rho) .
$$


If we take the spectral decomposition of the density matrix $\rho$, as $\rho=\sum_{i} \lambda_{i}\left|v_{i}\right\rangle\left\langle v_{i}\right|$, where the $\lambda_{i} \in[0,1]$ are the eigenvalues and the $\left|v_{i}\right\rangle \in \mathcal{H}$ are the (orthonormal) eigenvectors, then we get the standard Born rule:

$$
\left(\omega_{\rho} \models E\right)=\operatorname{tr}(E \rho)=\sum_{i} \lambda_{i}\left\langle v_{i}|E| v_{i}\right\rangle .
$$

What we use is the isomorphism between states and density matrices (for finite-dimensional $\mathcal{H})$ :

$$
\operatorname{Stat}(\mathcal{B}(\mathcal{H})) \cong \mathcal{D M}(\mathcal{H})
$$

At this stage we emphasise how much structure we get from the relatively mild effectus requirements in Assumption 1; we do not only have state and effect functors, but, if we involve the adjunction $\left(\mathbf{E M o d}_{M}\right)^{\mathrm{op}} \leftrightarrows \mathbf{C o n v}_{M}$ between effect modules and convex sets from Proposition 2.6, we also get a "state-and-effect" triangle of the form:

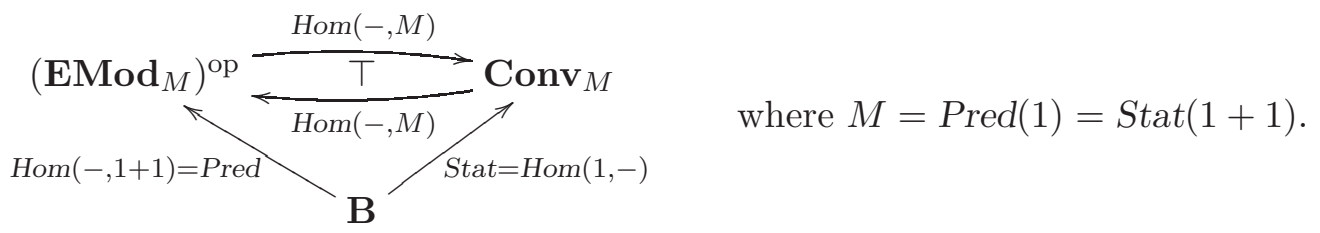

Such triangles are quite common in program semantics see [42, 43] for more information. They capture the correspondence between programs, in the base category, state transformers in the upper right category (in Schrödinger style), and predicate transformers in the upper left category (in Heisenberg style).

In general, the two triangles in (5.4) do not commute, but we do have canonical natural transformations between them, given by validity $=$.

Proposition 5.5. For an effectus $\boldsymbol{B}$ there are, in the setting of diagram (5.4), natural transformations:

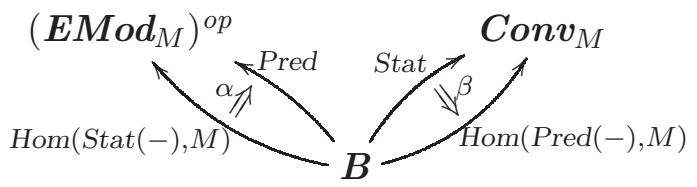

given as follows. For a predicate $p: X \rightarrow 1+1$ and state $\omega: 1 \rightarrow X$, both $\alpha$ and $\beta$ are defined via validity $\models$, as in:

$$
\alpha_{X}(p)(\omega)=\omega \models p=p \circ \omega=\beta_{X}(\omega)(p) .
$$

The maps $\alpha_{X}$ and $\beta_{X}$ are each other's transposes in the adjunction from Proposition 2.6:

$$
\frac{\operatorname{Pred}(X) \stackrel{\alpha_{X}}{\longrightarrow} \operatorname{Hom}(\operatorname{Stat}(X), M)}{\operatorname{Stat}(X) \underset{\beta_{X}}{\longrightarrow} \operatorname{Hom}(\operatorname{Pred}(X), M)}
$$

Note the reversal of direction for $\alpha$, due to the use of the opposite of the category of effect modules in (5.5). These $\alpha$ and $\beta$ may be called the Born natural transformations because they are given by the Born rule (5.1). 
Proof. It is easy to see that $\alpha$ and $\beta$ are natural transformations. Some basic properties have to be checked. For instance, each $\alpha_{X}(p)=p \circ(-)=p_{*}: \operatorname{Stat}(X) \rightarrow \operatorname{Stat}(1+1)=M$ is an affine map, by Lemma 5.2. And each $\alpha_{X}: \operatorname{Pred}(X) \rightarrow \operatorname{Hom}(\operatorname{Stat}(X), M)$ is a map of effect modules, where the homset inherits the effect module structure from $M=\operatorname{Pred}(1)$ in a pointwise manner, see Propositions 2.6 and 4.6. Similarly for $\beta$.

The triangle (5.4) commutes up-to-isomorphism when these maps $\alpha, \beta$ from (5.5) are both isomorphisms. This requires further assumptions, which we briefly illustrate (but do not impose). In our two main examples (with $M=[0,1]$ ) we do not have such commutation:
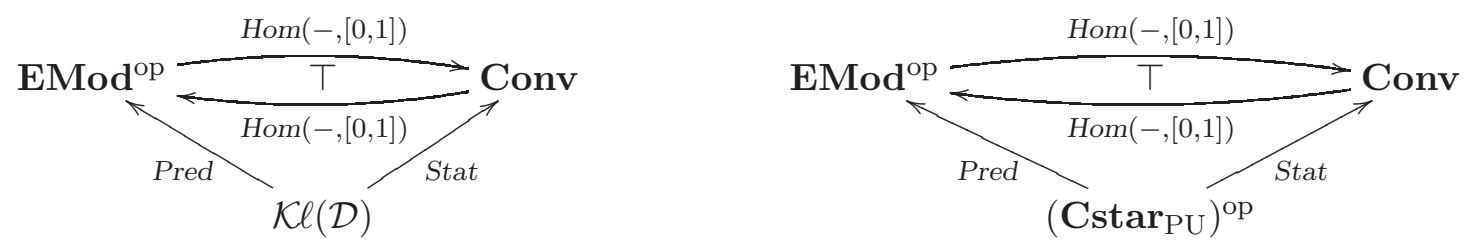

In the triangle on the left we do have:

$$
\operatorname{Hom}(\operatorname{Stat}(X),[0,1])=\operatorname{Conv}(\mathcal{D}(X),[0,1]) \cong[0,1]^{X}=\operatorname{Pred}(X) .
$$

But in the other direction we have $\operatorname{Hom}(\operatorname{Pred}(X),[0,1]) \cong \mathcal{D}(X)$ when $X$ is finite. For details, see [45], where the mapping $X \mapsto \operatorname{Hom}(\operatorname{Pred}(X),[0,1])=\operatorname{EMod}\left([0,1]^{X},[0,1]\right)$ is called the expectation monad $\mathcal{E}$. This expectation monad is a monad on Sets that extends the distribution monad, in the sense that $\mathcal{E}(X) \cong \mathcal{D}(X)$ for finite $X$.

For the $C^{*}$-algebra example on the right we do have commutation in the other direction:

$$
\operatorname{Hom}(\operatorname{Pred}(A),[0,1])=\operatorname{EMod}\left([0,1]_{A},[0,1]_{\mathbb{C}}\right) \cong \operatorname{Cstar}_{\mathrm{PU}}(A, \mathbb{C})=\operatorname{Stat}(A),
$$

using that the effect functor $[0,1]_{(-)}: \mathbf{C s t a r}_{\mathrm{PU}} \rightarrow \mathbf{E M o d}$ is full and faithful, see [26]. In the other direction we need to involve the (compact Hausdorff) topology on states of a $C^{*}$-algebra. Via the validity relation $\mid=$ one can put a weak-*-like topology on the homset of states. The restriction to convex compact Hausdorff spaces gives an equivalence with suitably complete effect modules, which is sometimes called Kadison duality after [49], see also [45].

A natural question is to which extent effects and states determine each other.

Definition 5.6. In an effectus B we say:

(1) predicates can be separated if for each pair of predicates $p_{1}, p_{2}: X \rightarrow 1+1$,

$$
\left(\forall \omega: 1 \rightarrow X .\left(\omega=p_{1}\right)=\left(\omega=p_{2}\right)\right) \Longrightarrow p_{1}=p_{2}
$$

Equivalently, if $p_{1} \neq p_{2}$, then there is a state $\omega$ with $\left(\omega \models p_{1}\right) \neq\left(\omega \models p_{2}\right)$. This expresses that the Born natural transformation $\alpha$ in (5.5) is injective.

(2) states can be separated if for each pair of states $\omega_{1}, \omega_{2}: 1 \rightarrow X$,

$$
\left(\forall p: X \rightarrow 1+1 .\left(\omega_{1} \models p\right)=\left(\omega_{2} \models p\right)\right) \Longrightarrow \omega_{1}=\omega_{2} .
$$

This says that $\beta$ in (5.5) is injective.

Separation of predicates is the more interesting property. For instance for distributive lattices it says that for (complementable) elements $a, b$ in a distributive lattice with $a \neq b$, there is a prime filter (or ideal) that contains one, but not the other. This separation property holds, see [48, I, Prop. 2.5] using the Prime Ideal Theorem. 
In $C^{*}$-algebras predicates can also be separated: let $a, b \in[0,1]_{A}$ be effects in a $C^{*}$ algebra $A$ with $a \neq b$. Then $a-b$ is a non-zero self-adjoint element. Hence by [6, Thm. 7.1.2] there is a state $\omega: A \rightarrow \mathbb{C}$ with $|\omega(a-b)|=\|a-b\|$. From $a-b \neq 0$ we get $\|a-b\| \neq 0$, and thus $\omega(a) \neq \omega(b)$.

Separation of states is an easier property. For instance, if we have two states $\omega_{1}, \omega_{2} \in$ $\mathcal{G}(X)$ in the Kleisli category $\mathcal{K} \ell(\mathcal{G})$ of the Giry monad with $\left(\omega_{1} \models p\right)=\left(\omega_{2} \models p\right)$ for each predicate $p$, then we take for $p$ indicator functions $\mathbf{1}_{M}: X \rightarrow[0,1]$ for $M \in \Sigma_{X}$. We get by (5.2):

$$
\omega_{1}(M)=\int \mathbf{1}_{M} \mathrm{~d} \omega_{1}=\left(\omega_{1}=\mathbf{1}_{M}\right)=\left(\omega_{2}=\mathbf{1}_{M}\right)=\int \mathbf{1}_{M} \mathrm{~d} \omega_{2}=\omega_{2}(M) .
$$

Hence $\omega_{1}=\omega_{2}$. Also, states can be separated in $C^{*}$-algebras: let $\omega_{1}, \omega_{2}: A \rightarrow \mathbb{C}$ be two states of a $\mathbb{C}$-algebra $A$ with $\omega_{1} \neq \omega_{2}$. Then there is an element $a \in A$ with $\omega_{1}(a) \neq \omega_{2}(a)$. Since each element in a $C^{*}$-algebra can be written as a linear combination of (four) positive elements - see also the proof of Lemma 8.6 later on - we may assume $a$ is positive (and $a \neq 0)$. We take $e=\frac{1}{\|a\|} \cdot a$, which is an effect since $0 \leq e \leq\|e\| \cdot 1=1$. Moreover,

$$
\left(\omega_{1}=e\right)=\omega_{1}(e)=\frac{1}{\|a\|} \cdot \omega_{1}(a) \neq \frac{1}{\|a\|} \cdot \omega_{2}(a)=\omega_{2}(e)=\left(\omega_{2} \models e\right) .
$$

Separation of predicates and/or states is thus a sensible additional requirement to add in axiomatisation.

5.1. Programs, states, and predicates. Let's take a closer look at the state-and-effect triangle (5.4) of an effectus B. We use the following interpretations of morphisms in $\mathbf{B}$.

$$
\begin{cases}\text { states } & \omega: 1 \longrightarrow X \\ \text { programs } & f: X \longrightarrow Y \\ \text { predicates } & q: Y \longrightarrow 1+1\end{cases}
$$

Such a program, also called a computation, $f: X \rightarrow Y$ yields two 'transformer' maps:

$$
\begin{cases}\text { state transformer } & f_{*}=f \circ(-): \operatorname{Stat}(X) \longrightarrow \operatorname{Stat}(Y) \\ \text { predicate transformer } & f^{*}=(-) \circ f: \operatorname{Pred}(Y) \longrightarrow \operatorname{Pred}(X)\end{cases}
$$

In a quantum setting the state transformer $f_{*}$ captures Schrödinger's style of forward computation, whereas the predicate transformer $f^{*}$, going backwards, corresponds to Heisenberg's style. It turns a predicate on the codomain of $f$ into a predicate on the domain of $f$, acting like a weakest precondition operation $w p(f)$.

Notice that the scalars $\omega \models f^{*}(q)$ and $f_{*}(\omega) \models q$ are the same, giving a Galois/adjoint style correspondence:

$$
\left(\omega \models f^{*}(q)\right)=(1 \stackrel{\omega}{\longrightarrow} X \stackrel{f}{\longrightarrow} Y \stackrel{q}{\longrightarrow} 1+1)=\left(f_{*}(\omega) \models q\right) .
$$

These scalars $\left(f_{*}(\omega) \models q\right)=\left(\omega \models f^{*}(q)\right)$ are interpreted as the expected probability that predicate $q$ holds after running program $f$ in state $\omega$. This Galois correspondence (5.7) is described more concretely for $\mathcal{K} \ell(\mathcal{G})$ in [40, Prop. 6], where it relates Kleisli composition and substitution for $\mathcal{G}$. This correspondence is reminiscent of the 'satisfaction condition' of institutions, see [30].

When the base category $\mathbf{B}$ in the triangle (5.4) is $\left(\mathbf{C s t a r}_{\mathrm{PU}}\right)^{\text {op }}$ the interpretation of the direction of computations is more subtle, because the category $\left(\text { Cstar }_{\mathrm{PU}}\right)^{\mathrm{op}}$ already captures Heisenberg's picture of quantum computation - also known as matrix mechanics. 
Its restriction to observables may be understood as a quantum version of weakest precondition calculation, see [21]. State transformer semantics for Hilbert spaces, considered as $C^{*}$-algebras via their bounded maps $\mathcal{B}(-)$, can be described as transformations of density matrices, via (5.3).

In the remainder of this paper we shall describe several constructions on programs and on predicates. Ideally, these constructions should come with appropriate calculation rules that break down the constructions to calculations with scalars/probabilities. For instance we already know that substitution maps $f^{*}$ preserve the effect module structure. In particular, $(\omega \models(-))=\omega^{*}: \operatorname{Pred}(X) \rightarrow \operatorname{Pred}(1)$ is a map of effect modules. Hence we can reduce validity probabilities $=$ involving $\oslash$ and $(-)^{\perp}$ on predicates to $\oslash$ and $(-)^{\perp}$ on scalars, as in:

$$
\begin{aligned}
\left(\omega \models f^{*}\left(q_{1} \otimes q_{2}\right)\right) & =\left(\omega \models f^{*}\left(q_{1}\right)\right) \otimes\left(\omega \models f^{*}\left(q_{2}\right)\right) \\
\left(\omega \models f^{*}\left(q^{\perp}\right)\right) & =\left(\omega \models f^{*}(q)\right)^{\perp} .
\end{aligned}
$$

\section{Predichtes and coproducts}

This section investigates the interaction in an effectus between coproducts, as in Assumption 11, and predicates. The elements of an effect module may be seen as formulas in a logic. There are truth and falsum formulas 1,0, and formulas are closed under sum $\oslash$ and orthocomplement $(-)^{\perp}$. In the current categorical context where we have effect modules $\operatorname{Pred}(X)$ of predicates $X \rightarrow 1+1$ on an object $X$ in a category, we can form additional formulas. Notably, if we have two predicates $p: X \rightarrow 1+1$ and $q: Y \rightarrow 1+1$ we can form a cotuple predicate $[p, q]: X+Y \rightarrow 1+1$. This cotuple looks like a new predicate constructor. But the isomorphism (6.1) in the next result shows that we get nothing new.

Lemma 6.1. Let $\boldsymbol{B}$ be an effectus, with its predicate functor Pred: $\boldsymbol{B} \rightarrow\left(\boldsymbol{E M o d}_{M}\right)^{\text {op }}$ from Proposition 4.6, where $M=\operatorname{Pred}(1)=\operatorname{Hom}(1,1+1)$ is the effect monoid of scalars. This functor Pred preserves finite coproducts and the final object 1, i.e. it sends:

- coproducts $(+, 0)$ in $\boldsymbol{B}$ to products $(\times, 1)$ in $\boldsymbol{E M o d}_{M}$;

- the final object $1 \in \boldsymbol{B}$ to the initial object $M=\operatorname{Pred}(1)$ in $\boldsymbol{E M}_{\boldsymbol{M}}$.

In particular, for objects $X, Y \in \boldsymbol{B}$ there is an isomorphism of effect modules:

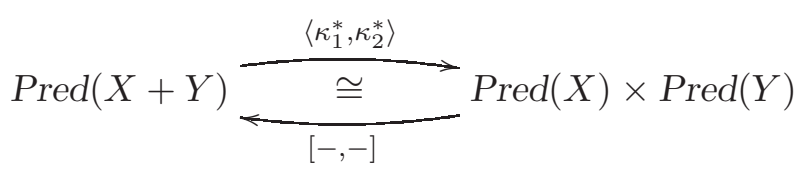

On the right-hand-side we use the obvious componentwise effect module structure. This isomorphism is natural in $X, Y$.

Proof. Clearly, $\operatorname{Pred}(0)=\operatorname{Hom}(0,1+1)$ is a singleton set, which, understood as trivial effect module, is final in the category $\mathbf{E M o d}_{M}$. The two functions $\left\langle\kappa_{1}^{*}, \kappa_{2}^{*}\right\rangle$ and $[-,-]$ in (6.1) are each other's inverses. We have already seen in Proposition 4.6 that substitution functors in particular these $\kappa_{i}^{*}$ - preserve the effect module structure. So we only have to prove that the cotuple map $[-,-]: \operatorname{Pred}(X) \times \operatorname{Pred}(Y) \rightarrow \operatorname{Pred}(X+Y)$ preserves the effect module structure.

Obviously, $[-,-]$ preserves truth:

$$
[1,1]=\left[\kappa_{1} \circ !_{X}, \kappa_{1} \circ !_{Y}\right]=\kappa_{1} \circ\left[!_{X}, !_{Y}\right]=\kappa_{1} \circ !_{X+Y}=1 .
$$


If we have $p_{1} \perp p_{2}$ for $p_{i} \in \operatorname{Pred}(X)$ via bound $b$, and $q_{1} \perp q_{2}$ for $q_{i} \in \operatorname{Pred}(Y)$ via bound $c$, then one easily checks that $[b, c]: X+Y \rightarrow(1+1)+1$ proves $\left[p_{1}, q_{1}\right] \perp\left[p_{2}, q_{2}\right]$ and also $\left[p_{1}, q_{1}\right] \otimes\left[p_{2}, q_{2}\right]=\left[p_{1} \otimes p_{2}, q_{1} \otimes q_{2}\right]$. Preservation of scalar multiplication from (4.7) is obvious:

$$
s \bullet[p, q]=\left[s, \kappa_{2}\right] \circ[p, q]=\left[\left[s, \kappa_{2}\right] \circ p,\left[s, \kappa_{2}\right] \circ q\right]=[s \bullet p, s \bullet q] .
$$

Naturality of the isomorphism in (6.1) is easy and left to the reader. Finally, Pred $(1)=M$ is clearly an initial object in $\operatorname{EMod}_{M}$, because for each $E \in \operatorname{EMod}_{M}$ there is a unique map $M \rightarrow E$ of effect modules, namely $r \mapsto r \bullet 1$.

The isomorphism (6.1) is known from many instances.

- In Sets it amounts to $\{0,1\}^{X+Y} \cong\{0,1\}^{X} \times\{0,1\}^{Y}$, which, in terms of powersets instead of characteristic functions becomes: $\mathcal{P}(X+Y) \cong \mathcal{P}(X) \times \mathcal{P}(Y)$.

- In $\mathcal{K} \ell(\mathcal{D})$ it means $[0,1]^{X+Y} \cong[0,1]^{X} \times[0,1]^{Y}$.

- And for $C^{*}$-algebras $A, B$ it says $[0,1]_{A \oplus B} \cong[0,1]_{A} \times[0,1]_{B}$.

Because Pred: $\mathbf{B} \rightarrow\left(\mathbf{E M o d}_{M}\right)^{\text {op }}$ preserves finite coproducts we have isomorphisms of effect modules:

$$
\operatorname{Pred}\left(X_{1}+\cdots+X_{n}\right) \cong \operatorname{Pred}\left(X_{1}\right) \times \cdots \times \operatorname{Pred}\left(X_{n}\right) .
$$

In particular, the right-to-left cotuple is a map of effect modules. This yields a useful equation:

$$
\left[p_{1}, \ldots, p_{n}\right] \otimes\left[q_{1}, \ldots, q_{n}\right]=\left[p_{1} \otimes q_{1}, \ldots, p_{n} \otimes q_{n}\right] .
$$

As immediate consequence we get $[p, q]=[p, 0] \otimes[0, q]$. The predicates $[p, 0]$ and $[0, q]$ occurring in this equation play a special role. Informally, the predicate $[p, 0]$ says that an element $z: X+Y$ must be in the first component $X$ of the coproduct $X+Y$ and in that case $p$ holds for $z$. The predicate $[p, 1]$ involves an implication: if $z$ is in the first component $X$, then $p$ holds for $z$. We define special operators:

$$
\operatorname{FstAnd}(p)=[p, 0] \quad \text { FstThen }(p)=[p, 1] .
$$

Similarly we have:

$$
\operatorname{SndAnd}(q)=[0, q] \quad \operatorname{SndThen}(p)=[1, q] .
$$

Lemma 6.1 implies $[p, q]=\operatorname{FstAnd}(p) \oplus \operatorname{Snd} A n d(q)$. It is easy to see that the "and" and "then" versions satisfy the De Morgan equalities:

$$
\operatorname{FstAnd}\left(p^{\perp}\right)^{\perp}=\operatorname{FstThen}(p) \quad \text { and } \quad \operatorname{SndAnd}\left(p^{\perp}\right)^{\perp}=\operatorname{SndThen}(p) .
$$

The relevance of these operators lies in the following result. It states that substitution functors $\kappa_{i}^{*}$ for coprojections automatically have adjoints. They will be useful later on in Section 9 .

Lemma 6.2. Let category $\boldsymbol{B}$ satisfy Assumption 1 . Then there are (order) adjunctions:

$$
\text { FstAnd } \dashv \kappa_{1}^{*} \dashv \text { FstThen } \quad \text { and } \quad \text { SndAnd } \dashv \kappa_{2}^{*} \dashv \text { SndThen }
$$

in a situation:

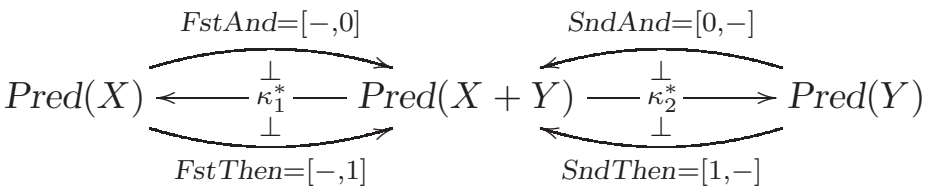


These adjoints commute with substitution, in the sense that for each map $f: Z \rightarrow X$ in $\boldsymbol{B}$ the following diagrams commute.
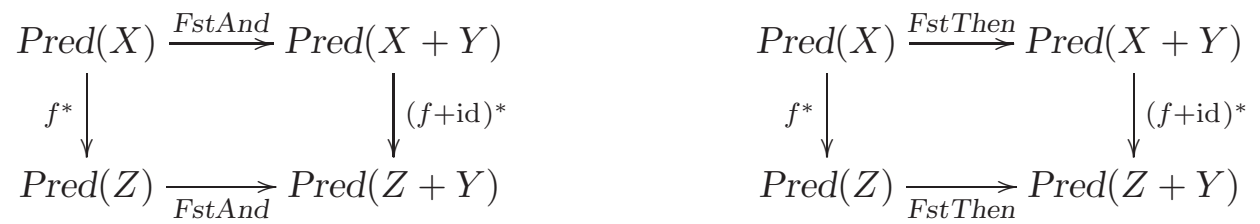

(And similarly for Snd.) In the language of fibrations - see [37] - this means that these adjoints form a fibred adjunction.

The adjoints in (6.4) are order adjoints (or Galois connections). In particular, the adjoints to $\kappa_{i}^{*}$ are not themselves maps of effect modules, but only monotone maps. Their properties will be described in greater detail in Lemma 6.3 below. In the categorical logic left and right adjoints to substitution are often written as $\amalg$ and $\prod$ respectively, see [37. Hence one may also write FstAnd $=\coprod_{\kappa_{1}}$ and FstThen $=\prod_{\kappa_{1}}$, and similarly for the second coprojection $\kappa_{2}$.

Proof. We prove that there is a bijective correspondence:

$$
\frac{\text { FstAnd }(p) \leq q}{p \leq \kappa_{1}^{*}(q)}
$$

where $q$ is a predicate on $X+Y$.

First, assume $\operatorname{FstAnd}(p) \leq q$, say via $\operatorname{FstAnd}(p) \oslash s=q$, where $s \in \operatorname{Pred}(X+Y)$. Let $b: X+Y \rightarrow(1+1)+1$ be a bound for the sum FstAnd $(p) \otimes s$, so that $I V \circ b=F$ stAnd $(p)$, $\mathrm{X} \circ b=s$, and $(\nabla+\mathrm{id}) \circ b=F \operatorname{FstAnd}(p) \oslash s=q$. We take $c=b \circ \kappa_{1}: X \rightarrow(1+1)+1$. Then one easily checks that $c$ is a bound showing $p \perp \kappa_{1}^{*}(s)$ and $p \oslash \kappa_{1}^{*}(s)=\kappa_{1}^{*}(q)$. Hence $p \leq \kappa_{1}^{*}(q)$.

In the other direction, assume $p \leq \kappa_{1}^{*}(q)$, via $p \oslash s=\kappa_{1}^{*}(q)$ with bound $b: X \rightarrow(1+1)+1$. We now take $c=\left[b,\left(\kappa_{2}+\mathrm{id}\right) \circ q \circ \kappa_{2}\right]: X+Y \rightarrow(1+1)+1$. Then $c$ proves FstAnd $(p) \perp t$, where $t=\left[s, q \circ \kappa_{2}\right]: X+Y \rightarrow 1+1$, and FstAnd $(p) \oslash t=q$. Hence FstAnd $(p) \leq q$.

Having established the adjunction FstAnd $\dashv \kappa_{1}^{*}$ we use the De Morgan equation to prove $\kappa_{1}^{*} \dashv$ FstThen in:

Finally, for $f: Z \rightarrow X$ one has:

$$
\begin{aligned}
& q \leq \operatorname{FstThen}(p)=\text { FstAnd }\left(p^{\perp}\right)^{\perp} \\
& \frac{\overline{\overline{\text { FstAnd }\left(p^{\perp}\right) \leq q^{\perp}}}}{\frac{p^{\perp} \leq \kappa_{1}^{*}\left(q^{\perp}\right)}{\overline{\kappa_{1}^{*}(q) \leq p}}} \kappa_{1}^{*}(q)^{\perp}
\end{aligned}
$$

$$
(f+\mathrm{id})^{*}(\operatorname{FstAnd}(p))=[p, 0] \circ(f+\mathrm{id})=[p \circ f, 0]=\left[f^{*}(p), 0\right]=\operatorname{FstAnd}\left(f^{*}(p)\right) .
$$

There is a bit more to say about these adjoints FstAnd and FstThen. We restrict ourselves to properties of FstAnd, because the corresponding properties for FstThen are easily obtained since FstThen can be expressed as the De Morgan dual of FstAnd, see (6.3). 
Lemma 6.3. The adjoint FstAnd $\dashv \kappa_{1}^{*}$ from Lemma 6.2 satisfies:

(1) $\kappa_{1}^{*} \circ$ FstAnd $=$ id;

(2) FstAnd preserves and reflects the order: $p \leq p^{\prime}$ iff FstAnd $(p) \leq$ FstAnd $\left(p^{\prime}\right)$;

(3) FstAnd(0) =0;

(4) FstAnd $\left(p_{1} \otimes p_{2}\right)=$ FstAnd $\left(p_{1}\right) \otimes$ FstAnd $\left(p_{2}\right)$, when $p_{1} \perp p_{2}$;

(5) FstAnd $(s \bullet p)=s \bullet$ FstAnd $(p)$ where $\bullet$ is scalar multiplication from (4.7).

Proof. The results follow from the isomorphism $\operatorname{Pred}(X+Y) \cong \operatorname{Pred}(X) \times \operatorname{Pred}(Y)$ in Lemma 6.1.

(1) Directly from the isomorphism 6.1.

(2) Idem, since the isomorphism preserves and reflects the order, so: $p \leq p^{\prime}$ iff $[p, 0] \leq\left[p^{\prime}, 0\right]$.

(3) Because (6.1) is an isomorphism of effect modules. Explicitly: FstAnd $\left(0_{X}\right)=\left[0_{X}, 0_{Y}\right]=$ $0_{X+Y}$.

(4) Similarly: FstAnd $\left(p_{1} \otimes p_{2}\right)=\left[p_{1} \otimes p_{2}, 0\right]=\left[p_{1} \otimes p_{2}, 0 \otimes 0\right]=\left[p_{1}, 0\right] \otimes\left[p_{2}, 0\right]=\operatorname{FstAnd}\left(p_{1}\right) \otimes$ FstAnd $\left(p_{2}\right)$.

(5) Idem, using that $s \bullet 0=0$.

Remark 6.4. As already mentioned, the coproducts + and the final object 1 in an effectus $\mathbf{B}$ give rise to the 'lift' or 'maybe' monad $\mathcal{L}=(-)+1$ on $\mathbf{B}$. Its unit maps $X \rightarrow X+1$ are the first coprojections $\kappa_{1}$, and its multiplication maps $(X+1)+1 \rightarrow X+1$ are of the form $\left[\mathrm{id}, \kappa_{2}\right]$. The associated category of Eilenberg-Moore algebras $\mathcal{E} \mathcal{M}(\mathcal{L})$ is isomorphic to the category with states $\omega: 1 \rightarrow X$ as object, corresponding to an algebra [id, $\omega]: X+1 \rightarrow X$. Maps correspond to commuting triangles between states.

The Kleisli category of the lift monad, written as $\mathbf{B}_{+1}$, typically captures partial computations that may fail to produce an output, for instance via non-terminating behaviour. The Kleisli category of the lift monad $(-)+1$ on Sets has maps of the form $X \rightarrow Y+1$, corresponding to partial functions $X \rightarrow Y$. With the 'flat' order, these maps form a directed complete partial order (dcpo).

We can also consider the lift monad $\mathcal{L}=(-)+1$ on the Kleisli category of the distribution monad $\mathcal{D}$. A map $X \rightarrow Y$ in the associated Kleisli category (of lift) is a map $X \rightarrow Y+1$ in the Kleisli category $\mathcal{K} \ell(\mathcal{D})$ of the distribution monad. Hence it is a function $X \rightarrow \mathcal{D}(Y+1)$. A crucial observations is that a distribution $\varphi \in \mathcal{D}(Y+1)$ on $Y+1$ is a subdistribution on $Y$ : a formal convex sum $\sum_{i} r_{i}\left|y_{i}\right\rangle$ of elements $y_{i} \in Y$ and probabilities $r_{i} \in[0,1]$ whose sum is below 1 , as in: $\sum_{i} r_{i} \leq 1$. These subdistributions can be ordered pointwise, and have the everywhere-zero subdistribution as least element. In case infinite supports are allowed, they are directed complete.

A similar phenomenon exists for $C^{*}$-algebras: the lift monad $(-) \oplus \mathbb{C}$ on the opposite

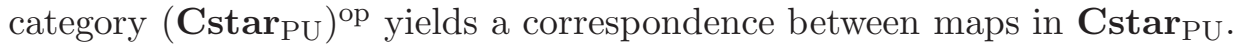

$$
\frac{\text { positive unital } A \oplus \mathbb{C} \stackrel{f}{\longrightarrow} B}{\text { positive subunital } A \underset{g}{\longrightarrow} B}
$$

Subunitality of $g: A \rightarrow B$ means $0 \leq g(1) \leq 1$. This correspondence is given as follows. For $f: A \oplus \mathbb{C} \rightarrow B$ take $\bar{f}(a)=f(a, 0)$, and for $g: A \rightarrow B$, take $\bar{g}(a, z)=g(a)+z \cdot(1-g(1))$. When $A, B$ are $W^{*}$-algebras, the normal completely positive subunital maps $A \rightarrow B$ carry a dcpo structure, see [15, 70, which can be used to interpret loops, like while or recursion, see also [71] for more information. 
A systematic investigation of partiality in effectuses can be found in [16]. There, the main result shows that an effectus can equivalently be described as a finitely partially additive category (as in [5]), with suitable effect algebra structure. This shows that partiality is an intrinsic feature of the theory of effectuses.

Remark 6.5. The starting point of this section is Lemma 6.1 showing that the predicate functor Pred: B $\rightarrow$ EMod $^{\mathrm{op}}$ of an effectus B automatically preserves coproducts. A natural question to ask is: does the states functor Stat: $\mathbf{B} \rightarrow \mathbf{C o n v}$ in a state-and-effect triangle (5.4) also preserve coproducts?

This matter is addressed in [47. There it is shown that preservation of + by the states functor Stat does not come for free, but corresponds to an important property, namely normalisation of states. This is relevant in conditional probability, as we briefly illustrate in the Kleisli category $\mathcal{K} \ell(\mathcal{D})$ of the distribution monad $\mathcal{D}$ on Sets, where this preservation/normalisation property holds. If we have a distribution $\varphi \in \mathcal{D}(X+Y)$ on a coproduct set $X+Y$, then we can write $\varphi$ as convex combination $r \cdot \mathcal{D}\left(\kappa_{1}\right)(\varphi \mid X)+(r-1) \cdot \mathcal{D}\left(\kappa_{2}\right)(\varphi \mid Y)$, for certain 'normalised' distributions $\varphi|X \in \mathcal{D}(X), \varphi| Y \in \mathcal{D}(Y)$ and $r=\sum_{x \in X} \varphi\left(\kappa_{1} x\right) \in[0,1]$. Such convex combinations form the coproduct in the category Conv, see [4] for details.

\section{Measurement instruments}

Our second assumption introduces (discrete) measurement instruments as certain maps in

our base category. Such instruments have been introduced in [20], see also [66] and [35]. Characteristic aspects are:

(1) different measurement outcomes can be distinguished

(2) state changes caused by measurement are taken into account.

Assumption 2 below describes our categorical formalisation of discrete instruments for finitary measurements, as maps of the form $X \rightarrow X+\cdots+X$, induced by a test. The original references [20, 66] deal with the non-finitary, continuous case, where effects are indexed via a measurable space, see Remark 7.3 (1) below. The different measurement outcomes in the first point correspond to the different coproduct options in the codomain $X+\cdots+X$. The state changes are captured by the side-effect associated with such an instrument, which we define as the composite with the codiagonal $\nabla=[\mathrm{id}, \cdots, \mathrm{id}]$ in:

$$
X \longrightarrow X+\cdots+X \stackrel{\nabla}{\longrightarrow} X
$$

There is no side-effect if this map $X \rightarrow X$ is the identity. We use the term 'side-effect-free' for this situation. These side-effects will be studied systematically in the next section. In this section we concentrate on the definition of instruments and on examples, and show how they can be used for guarded test programs.

We should emphasise that the instrument assumption below requires the presence of certain maps, satifying certain properties. It is an open question, under which conditions, these properties fully determine the instrument maps. Hence these instruments form structure in the category, and their presence is not a property of the category - as long as we cannot show that they are uniquely determined. This is not uncommon: our third assumption is about the presence of tensors $\otimes$, which are also non-uniquely determined structure. 
Assumption 2. Let $\mathbf{B}$ be an effectus, i.e. a category satisfying Assumption 1, For each $n$-test $p: X \rightarrow n \cdot 1$ there is a measurement instrument map instr $p: X \rightarrow n \cdot X$ in $\mathbf{B}$ making the following diagram commute.

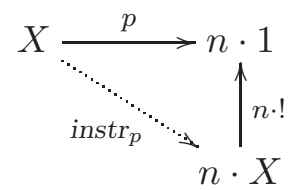

Notice that the ' 1 ' in the codomain of a test $X \rightarrow n \cdot 1$ is replaced by ' $X$ ' in an instrument $X \rightarrow n \cdot X$. Thus, where a test only captures the different output options (and no outcome, in 1), an instrument has both options and an outcome (in $X$ ). The above diagram says that annihilating these outcomes in $X$ via the unique map !: $X \rightarrow 1$ returns the original test.

We do not require uniqueness of these instruments, but we do require the following properties.

(1) (Injection) For an injective function $\phi:\{1, \ldots, n\} \longmapsto\{1, \ldots, m\}$ and an object $A$, write $[\phi]: n \cdot A \rightarrow m \cdot A$ for the obvious map $[\phi]=\left[\kappa_{\phi(1)}, \ldots, \kappa_{\phi(n)}\right]$. Then for each $n$-test $p: X \rightarrow n \cdot 1$ the following diagram commutes.

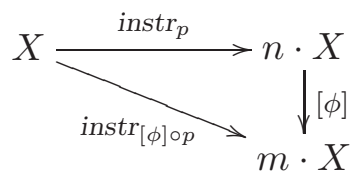

This property says that reordering elements of a test and adding zero (falsum) predicates is reflected in the corresponding instrument. It implies for instance: instr $p_{p^{\perp}}=\left[\kappa_{2}, \kappa_{1}\right] \circ$ instr $r_{p}$, for a predicate $p: X \rightarrow 1+1$, where $p^{\perp}=\left[\kappa_{2}, \kappa_{1}\right] \circ p$ is the orthocomplement.

(2) (Cotuple) For two $n$-tests $p: X \rightarrow n \cdot 1$ and $q: Y \rightarrow n \cdot 1$, the following diagram commutes:

$$
\begin{aligned}
X+Y \underset{\text { instr }_{p}+\text { instr }_{q}}{\stackrel{\operatorname{instr}_{[p, q]}}{\longrightarrow}} & n \cdot(X+Y) \\
& \cong \downarrow\left[\kappa_{1}+\kappa_{1}, \ldots, \kappa_{n}+\kappa_{n}\right] \\
n & =X+n \cdot Y
\end{aligned}
$$

(3) (Collapse) For each $n+m$-test $p: X \rightarrow n \cdot 1+m \cdot 1$ one can "collapse" the $m$-options in two different ways, but this gives the same outcome, as expressed by the following commuting diagram.

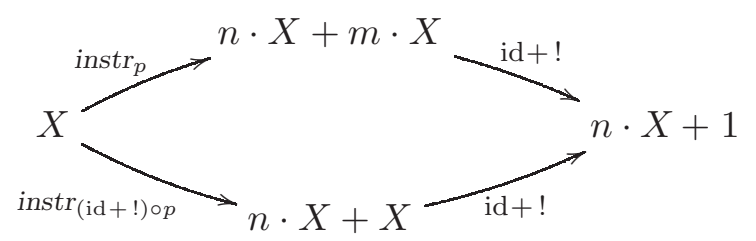

(4) (Side-effect-free) Given a map $q: X \rightarrow n \cdot X$ in $\mathbf{B}$ which is side-effect-free, that is $\nabla \circ q=\mathrm{id}$, then $q=$ instr $_{p}$ for the $n$-test $p=(n \cdot !) \circ q: X \rightarrow n \cdot 1$. In diagrams:

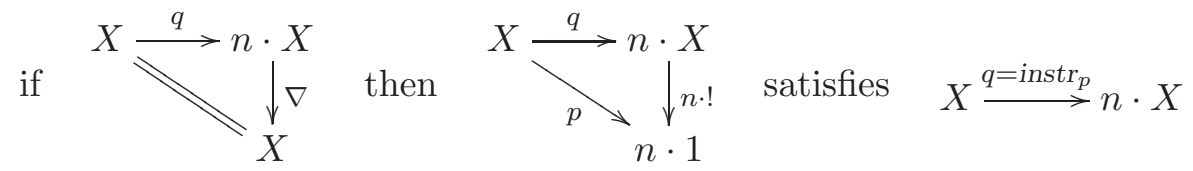


When $\nabla \circ q=$ id as in the last property, then $q: X \rightarrow X+\cdots+X$ is merely selecting paths among the various coproduct options, without having any computational effect. Therefor we call such a $q$ side-effect-free, see the next section. The above property (4) says that such path-selector maps $q$ arise as instruments from the corresponding path-selector test $(n \cdot !) \circ q: X \rightarrow 1+\cdots+1$. This path property is non-trivial, especially for $C^{*}$-algebras, see Corollary 8.5. We explicitly mention two consequences.

Lemma 7.1. In a category satisfying Assumption 2 we have:

(1) instr $_{q}=q$ for each $n$-test $q: 1 \rightarrow n \cdot 1$ on the final object 1 ;

(2) instr $_{\kappa_{i} \circ !}=\kappa_{i}: X \rightarrow n \cdot X$, for each $1 \leq i \leq n$.

In particular, $\operatorname{instr}_{q}=q$ when $q$ is a scalar, and instr ${ }_{1}=\kappa_{1}$, instr $_{0}=\kappa_{2}$, for the true and false predicates $1,0: X \rightarrow 1+1$.

Proof. We use property (44) from Assumption 2 in both cases. For $q: 1 \rightarrow n \cdot 1$ we clearly have $\nabla \circ q=$ id by finality of 1 . But also $p=(n \cdot !) \circ q=q$, so that $\operatorname{instr}_{q}=q$. For the second point, notice that the coprojection $\kappa_{i}: X \rightarrow n \cdot X$ satisfies $\nabla \circ \kappa_{i}=$ id. The $n$-test $\left(n \cdot !_{X}\right) \circ \kappa_{i}=\kappa_{i} \circ !_{X}: X \rightarrow n \cdot 1$ gives instr $_{\kappa_{i} \circ !_{X}}=\kappa_{i}$.

We illustrate these instruments in our leading examples.

Examples 7.2. In the category Sets, an $n$-test $p: X \rightarrow n \cdot 1=\{1,2, \ldots, n\}$ corresponds to a partition of $X$, that is, to a cover of $X$ given by disjoint subsets. Hence we can define an instrument $\operatorname{instr}_{p}: X \rightarrow n \cdot X$ that picks the $i$-th coproduct option if the input $x$ is in the $i$-th subset:

$$
\operatorname{instr}_{p}(x)=\kappa_{i} x \quad \text { iff } \quad p(x)=i .
$$

Clearly, $n \cdot ! \circ$ instr $_{p}=p$.

For an $n$-test $p: X \rightarrow \mathcal{D}(n)$ in the Kleisli category $\mathcal{K} \ell(\mathcal{D})$ the associated instrument instr $_{p}: X \rightarrow \mathcal{D}(n \cdot X)$ works as before, but now gives a distribution over the various coproduct options, via the following convex sum:

$$
\operatorname{instr}_{p}(x)=p(x)(1)\left|\kappa_{1} x\right\rangle+\cdots+p(x)(n)\left|\kappa_{n} x\right\rangle
$$

In the case of continuous probability, given by the Kleisli category $\mathcal{K} \ell(\mathcal{G})$ of the Giry $\operatorname{monad} \mathcal{G}$, an $n$-test is a measurable map $p: X \rightarrow \mathcal{G}(n) \cong \mathcal{D}(n)$. Hence we can use basically the same construction as before: the instrument $\operatorname{instr}_{p}: X \rightarrow \mathcal{G}(n \cdot X)$ sends $x \in X$ to the probability measure $\operatorname{instr}_{p}(x): \Sigma_{n \cdot X} \rightarrow[0,1]$ defined on basic measurable subsets $\kappa_{i} M \in$ $\Sigma_{n \cdot X}$ as:

$$
\operatorname{instr}_{p}(x)\left(\kappa_{i} M\right)=p(x)(i) \cdot \mathbf{1}_{M}(x)= \begin{cases}p(x)(i) & \text { if } x \in M \\ 0 & \text { otherwise. }\end{cases}
$$

We sketch the proof of requirement (4) because it is slightly subtle. Let $q: X \rightarrow \mathcal{G}(n \cdot X)$ be a measurable map satisfying $\nabla \odot q=$ id, where $\odot$ is Kleisli composition. One easily checks that this amounts to, for $x \in X$ and $M \in \Sigma_{X}$,

$$
\mathbf{1}_{M}(x)=\eta(x)(M)=\mathrm{id}(x)(M)=q(x)\left(\kappa_{1} M\right)+\cdots+q(x)\left(\kappa_{n} M\right)
$$

This equality gives some useful information. First, if $x \notin M$, then $\mathbf{1}_{M}(x)=0$, so $q(x)\left(\kappa_{i} M\right)=$ 0 for each $i$. Second, if $M \subseteq N$ then $q(x)\left(\kappa_{i} M\right) \leq q(x)\left(\kappa_{i} N\right)$ by monotonicity of the probability measure $q(x)$. But if $x \in M$ this $\leq$ must be an equality, since also $1=\mathbf{1}_{N}(x)=$ $q(x)\left(\kappa_{1} N\right)+\cdots+q(x)\left(\kappa_{n} N\right)$. Third, $q(x)\left(\kappa_{i} M\right)=q(x)\left(\kappa_{i} X\right) \cdot \mathbf{1}_{M}(x)$. Indeed, if $x \notin M$ 
then both sides are 0 by the first observation. And if $x \in M$, then both sides are equal by the second one. Next we note that the test $(n \cdot !) \odot q: X \rightarrow \mathcal{G}(n)$ can be described as:

$$
((n \cdot !) \odot q)(x)(i)=\int(n \cdot !)(-)(i) \mathrm{d} q(x)=\int \mathbf{1}_{\kappa_{i} X} \mathrm{~d} q(x)=q(x)\left(\kappa_{i} X\right) .
$$

Finally we can prove $\operatorname{instr}_{(n \cdot !) \odot q}=q$ via:

$$
\operatorname{instr}_{(n ! !) \odot q}(x)\left(\kappa_{i} M\right)=((n \cdot !) \odot q)(x)(i) \cdot \mathbf{1}_{M}(x)=q(x)\left(\kappa_{i} X\right) \cdot \mathbf{1}_{M}(x)=q(x)\left(\kappa_{i} M\right) .
$$

We continue with rings and show that instruments exist for commutative rings. For a ring $R$ an $n$-test $R \rightarrow n \cdot 1$ consists of $n$ idempotents $\vec{e}=e_{1}, \ldots, e_{n} \in R$ with $e_{1}+\cdots+e_{n}=1$ and $e_{i} \cdot e_{j}=0$ for $i \neq j$. If $R$ is commutative we get an instrument $\operatorname{instr}_{\vec{e}}$ : $R \rightarrow n \cdot R$ in the opposite category $\mathbf{C R n g}^{\mathrm{op}}$. In the category $\mathbf{C R n g}$ it takes the form of a function instr $r_{\vec{e}}: R^{n} \rightarrow R$ defined by:

$$
\operatorname{instr}_{\vec{e}}\left(x_{1}, \ldots, x_{n}\right)=e_{1} \cdot x_{1}+\cdots+e_{n} \cdot x_{n} .
$$

It clearly preserves sums $(+, 0)$. It also preserves multiplications $(\cdot, 1)$ by the properties of an $n$-test and commutativity. Of the required properties in Assumption 2 we only do number (44): if $q: R^{n} \rightarrow R$ is a 'side-effect-free' ring homomorphism with $q \circ \Delta=\mathrm{id}$, then $q(x, \ldots, x)=x$. Moreover, the $n$-test $p=q \circ !^{n}: \mathbb{Z}^{n} \rightarrow R$ is given by $p\left(m_{1}, \ldots, m_{n}\right)=$ $q\left(m_{1} \cdot 1, \ldots, m_{n} \cdot 1\right)$, and corresponds to idempotents $e_{i}=q(|i\rangle) \in R$, satisfying:

$$
\begin{aligned}
\operatorname{instr}_{\vec{e}}\left(x_{1}, \ldots, x_{n}\right) & =e_{1} \cdot x_{1}+\cdots+e_{n} \cdot x_{n} \\
& =q(|1\rangle) \cdot q\left(x_{1}, \ldots, x_{1}\right)+\cdots+q(|n\rangle) \cdot q\left(x_{n}, \ldots, x_{n}\right) \\
& =q\left(|1\rangle \cdot\left(x_{1}, \ldots, x_{1}\right)\right)+\cdots+q\left(|n\rangle \cdot\left(x_{n}, \ldots, x_{n}\right)\right) \\
& =q\left(x_{1}, 0, \ldots, 0\right)+\cdots+q\left(0, \ldots, 0, x_{n}\right) \\
& =q\left(x_{1}, \ldots, x_{n}\right) .
\end{aligned}
$$

Along the same lines one shows that instruments exist in the opposite $\mathbf{D} \mathbf{L}^{\mathrm{op}}$ of the category of distributive lattices: for such a lattice $L$, with an $n$-test, consisting of elements $e_{1}, \ldots, e_{n} \in$ $L$ satisfying $e_{1} \vee \cdots \vee e_{n}=1$, and $e_{i} \wedge e_{j}=0$ for $i \neq j$, one defines an instrument $\operatorname{instr}_{\vec{e}}: L^{n} \rightarrow L$ by:

$$
\operatorname{instr}_{\vec{e}}\left(x_{1}, \ldots, x_{n}\right)=\left(e_{1} \wedge x_{1}\right) \vee \cdots \vee\left(e_{n} \wedge x_{n}\right) .
$$

We turn to $C^{*}$-algebras. In a $C^{*}$-algebra $A$, with an $n$-test $\vec{e}=e_{1}, \ldots, e_{n} \in[0,1]_{A}$ where $e_{1}+\cdots+e_{n}=1$, we have an instrument instr $\vec{e}_{\vec{e}}: A^{n} \rightarrow A$ given by:

$$
\operatorname{instr}_{\vec{e}}\left(a_{1}, \ldots, a_{n}\right)=\sqrt{e_{1}} \cdot a_{1} \cdot \sqrt{e_{1}}+\cdots+\sqrt{e_{n}} \cdot a_{n} \cdot \sqrt{e_{n}} .
$$

This map is not only positive, but completely positive. We notice that if the $C^{*}$-algebra $A$ is commutative, then $\operatorname{instr}_{\vec{e}}\left(a_{1}, \ldots, a_{n}\right)=e_{1} \cdot a_{1}+\cdots+e_{n} \cdot a_{n}$, like for rings in (7.4). This formula (7.5) is sometimes called the (generalised) Lüders rule, for unsharp predicates (effects). It can be found, for instance, in [12, Eq.(1.3)].

Of the different properties (1)-(4) in Assumption 2 for instruments we shall demonstrate (3). So assume we have an $n+m$-test, of the form $p: \mathbb{C}^{n} \times \mathbb{C}^{m} \rightarrow A$. It corresponds to $n+m$ effects $e_{1}, \ldots, e_{n}, d_{1}, \ldots, d_{m} \in[0,1]_{A}$, where $e_{i}=p(|i\rangle, \overrightarrow{0})$ and $d_{j}=p(\overrightarrow{0},|j\rangle)$, with $\sum_{i} e_{i}+\sum_{j} d_{j}=1$. The test $p^{\prime}=p \circ(\mathrm{id} \times !): \mathbb{C}^{n} \times \mathbb{C} \rightarrow A$ is given by $p^{\prime}\left(z_{1}, \ldots, z_{n}, w\right)=$ $p\left(z_{1}, \ldots, z_{n}, w, \ldots, w\right)$. Hence $p^{\prime}$ corresponds to the $n+1$-tuple of effects $e_{1}, \ldots, e_{n}, \sum_{j} d_{j}$. 
We can now compute the outcomes of the two paths in the diagram in Assumption 2 (3) in the category Cstar $_{\mathrm{PU}}$. We show that the two associated maps $A^{n} \times \mathbb{C} \rightarrow A$ are equal. The upper composite is:

$$
\begin{aligned}
\left(\operatorname{instr}_{p} \circ(\operatorname{id} \times !)\right)\left(a_{1}, \ldots, a_{n}, w\right) & =\operatorname{instr}_{\vec{e}, \vec{d}}\left(a_{1}, \ldots, a_{n}, w \cdot 1, \ldots, w \cdot 1\right) \\
& =\left(\sum_{i} \sqrt{e_{i}} \cdot a_{i} \cdot \sqrt{e_{i}}\right)+\left(\sum_{j} \sqrt{d_{j}} \cdot(w \cdot 1) \cdot \sqrt{d_{j}}\right) \\
& =\left(\sum_{i} \sqrt{e_{i}} \cdot a_{i} \cdot \sqrt{e_{i}}\right)+w \cdot\left(\sum_{j} d_{j}\right) .
\end{aligned}
$$

We get the same outcome if we follow the lower path:

$$
\begin{aligned}
\left(\operatorname{instr}_{p^{\prime}} \circ(\operatorname{id} \times !)\right)\left(a_{1}, \ldots, a_{n}, w\right) & =\operatorname{instr}_{\vec{e}, \sum_{j} d_{j}}\left(a_{1}, \ldots, a_{n}, w \cdot 1\right) \\
& =\left(\sum_{i} \sqrt{e_{i}} \cdot a_{i} \cdot \sqrt{e_{i}}\right)+\left(\sqrt{\sum_{j} d_{j}} \cdot(w \cdot 1) \cdot \sqrt{\sum_{j} d_{j}}\right) \\
& =\left(\sum_{i} \sqrt{e_{i}} \cdot a_{i} \cdot \sqrt{e_{i}}\right)+w \cdot\left(\sum_{j} d_{j}\right) .
\end{aligned}
$$

The verification of property (44) for $C^{*}$-algebras will be postponed to Corollary 8.5 in the next section.

We briefly consider the special cases where the $C^{*}$-algebra is $\mathcal{B}(\mathcal{H})$, for a Hilbert space $\mathcal{H}$. The effects $[0,1]_{\mathcal{B}(\mathcal{H})}$ of $\mathcal{B}(\mathcal{H})$ are the positive maps $\mathcal{H} \rightarrow \mathcal{H}$ below the identity. Suppose our $n$-test of effects $p_{1}, \ldots, p_{n} \in[0,1]_{A}$ consists of projections: $p_{i}^{2}=p_{i}$. Then $\sqrt{p_{i}}=p_{i}$. Hence the associated instrument instr $\vec{e}: \mathcal{B}(\mathcal{H})^{n} \rightarrow \mathcal{B}(\mathcal{H})$ takes the familiar form:

$$
\operatorname{instr}_{\vec{p}}\left(A_{1}, \ldots, A_{n}\right)=p_{1} A_{1} p_{1}+\cdots p_{n} A_{n} p_{n} .
$$

Remark 7.3. The above list of examples gives rise to the following observations.

(1) For an $n$-test $\vec{e}$ in a $C^{*}$-algebra $A$ we have an instrument $\operatorname{instr}_{\vec{e}}\left(a_{1}, \ldots, a_{n}\right)=\sum_{i} \sqrt{e_{i}}$. $a_{1} \cdot \sqrt{e_{i}}$ as defined in (7.5). We briefly show how it forms a (discrete) instrument as defined in [66, §4], see also [35, §5.1.2]. The map instr $\vec{e}_{\vec{e}}$ can be seen as a combination of $n$ completely positive subunital maps $A \rightarrow A$, namely $f_{i}(a)=\sqrt{e_{i}} \cdot a_{1} \cdot \sqrt{e_{i}}$. These maps $f_{i}$ are subunital since $0 \leq f_{i}(1)=e_{i} \leq 1$. In fact, let $\Sigma_{n}=\mathcal{P}(n)$ be the set of measurable subsets of the discrete measurable space $n$. Then we can define for each (measurable) subset $U \subseteq n$ a completely positive subunital map $f_{U}: A \rightarrow A$ via $f_{U}(a)=\sum_{i \in U} \sqrt{e_{i}} \cdot a_{1} \cdot \sqrt{e_{i}}$. As required for a (discrete) instrument, this mapping $U \mapsto f_{U}$ sends disjoint unions $U \otimes V$ to sums $f_{U}+f_{V}$. Moreover, the function $f_{n}$ associated with the largest (measurable) subset $n \subseteq n$ is unital.

Thus we have constructed a function from a $\sigma$-algebra $\Sigma$ to the completely positive subunital maps $A \rightarrow A$ that is additive, and sends the top element to a unital function. This is (the $C^{*}$-algebraic version of) an instrument as defined in [20, 66, 35].

(2) We have defined instruments in (7.4) only for commutative rings. For a non-commutative ring $R$ with an $n$-test $\vec{e}$ one can define a map $f: R^{n} \rightarrow R$ as $f\left(x_{1}, \ldots, x_{n}\right)=\sum_{i} e_{i} \cdot x_{i} \cdot e_{i}$. However, this map does not preserve multiplication. Hence one may try to adapt the notion of 'ring homomorphism' to something weaker, just like PU is a weakening of MIU for $C^{*}$-algebras. This requires a careful balancing act, because one would still like to have a reasonable notion of predicate as this new kind of map $R \rightarrow 1+1$. There is no obvious way to do that. This balancing act does work for $C^{*}$-algebras, by not using MIU but PU maps.

(3) We have used the computer science terminology 'side-effect' for what is more commonly called 'observer effect' in physics. It is an interesting question to what extent instruments, as defined above are useful to capture side-effects in computer science. Here is 
a first thought. One can imagine a database with state space $X$ for which one may observe certain content, say via a function $X \rightarrow n$, where what is observed is described here simply as a finite set $n$ of natural numbers. Associated with such an observer map $X \rightarrow n$ one can construct an 'instrument' $X \rightarrow n \cdot X$ which has a side-effect on the state space $X$ of the database, for instance by logging the observation itself. This can be done for accountability reasons, like in a medical database. The instrument used for observation thus changes the state of the database by adding a log entry.

(4) Assumption 2 lists four coherence requirements for instruments. They suffice in the present setting. However, we do not claim that these four requirements are 'complete'. Possibly, other formulations are more adequate, and guarantee that instruments are unique. This issue is still an area of active research. For instance, in [17] instruments are (equivalently) described via partial 'assert' maps $X \rightarrow X+1$, like in point (1). It is illustrated that in several example categories these assert maps can be obtained from quotient and comprehension maps, but the precise defining property remains elusive. Recent, unpublished research shows that in 'Boolean' and in 'classical' effectuses these assert/instrument maps are uniquely determined; but the 'quantum' case remains open.

We conclude this section by showing how instruments instr as in Assumption 2 give rise to a natural programming language construct, namely a general test map in the base category of computations: for an $n$-test $p: X \rightarrow n \cdot 1$ and $n$ parallel maps $f_{1}, \ldots, f_{n}: X \rightarrow Y$ we can define a test map $X \rightarrow Y$ as:

$$
p ?\left[f_{1}, \ldots, f_{n}\right]=\left(X \stackrel{\text { instr }_{p}}{\longrightarrow} n \cdot X=X+\cdots+X \stackrel{\left[f_{1}, \ldots, f_{n}\right]}{\longrightarrow} Y\right) .
$$

The idea is that this test map $p ?\left[f_{1}, \ldots, f_{n}\right]$ first performs a $p$-test, and then, depending on the outcome, does a suitable combination of the maps $f_{1}, \ldots, f_{n}$. This combination depends on the coproduct options in $n \cdot X$ that $p$ produces and acts on the state after $p$, incorporating the side-effect of $p$, if any.

Via the bijective correspondence (4.11) between an $n$-test $p: X \rightarrow n \cdot 1$ and $n$ separate predicates $p_{1}, \ldots, p_{n}: X \rightarrow 1+1$ with $p_{1} \otimes \cdots \otimes p_{n}=1$, we can understand this test map $p ?\left[f_{1}, \ldots, f_{n}\right]$ in (7.6) also as a guarded command in the style of [23], written alternatively as:

$$
\text { if } \begin{gathered}
\mid p_{1} \rightarrow f_{1} \\
\vdots \\
\text { fi } \mid p_{n} \rightarrow f_{n}
\end{gathered}
$$

or, as we shall write later, in Figure 4 .

$$
\begin{gathered}
\text { begin test } x \\
\qquad \begin{array}{c}
\mid p_{1} \rightarrow f_{1} \\
\vdots \\
\mid p_{n} \rightarrow f_{n}
\end{array} \\
\text { end test }
\end{gathered}
$$

In the set-theoretic case these $p_{i}$ describe a partition of $X$, so that for each $x \in X$ precisely one $f_{i}$ is selected. In the probabilistic case a suitable convex combination of the $f_{i}$ is executed. And in the quantum case this test map involves a more complicated combination using square roots of the effects, as in (7.5).

Appropriate logical "test" operators to reason about these guarded commands will be described in Section 9. At this stage we observe the following obvious property:

$$
g \circ\left(p ?\left[f_{1}, \ldots, f_{n}\right]\right)=p ?\left[g \circ f_{1}, \ldots, g \circ f_{n}\right] .
$$

A corresponding pre-composition property holds for so-called pure maps, see (8.6). 
The convex sum $\emptyset_{i} r_{i} \omega_{i}$ of states $\omega_{i}$ defined in the proof of Lemma 5.2 is an instance of this test programming construct (7.6):

$$
\begin{aligned}
& \emptyset_{i} r_{i} \omega_{i}=\left[\omega_{1}, \ldots, \omega_{n}\right] \circ p=\text { if } \\
& \mid r_{1} \rightarrow \omega_{1} \\
& \vdots \\
& \text { fi } \mid r_{n} \rightarrow \omega_{n}
\end{aligned}
$$

This follows from the equation $\operatorname{instr}_{p}=p$ for $p: 1 \rightarrow n \cdot 1$ in Lemma 7.1 (11).

\section{Side-effect free PREDicates AND Pure maps}

One of the advantages of the current, general approach is that it allows us to express the sideeffect - sometimes called the observer effect — of an instrument. We already encountered it briefly in the previous section. Here we add some further results about side-effect-freeness, notably in relation to commutativity in $C^{*}$-algebras. Also, we introduce an abstract notion of purity for maps.

Definition 8.1. Given a choice of mesurement instruments, the side-effect of an $n$-test $p: X \rightarrow n \cdot 1$ is the endomap $X \rightarrow X$ obtained in:

$$
X \stackrel{\text { instr }_{p}}{\longrightarrow} X+\cdots+X \stackrel{\nabla=[\mathrm{id}, \ldots, \mathrm{id}]}{\longrightarrow} X
$$

This test $p$ is called side-effect-free if its side-effect map $\nabla \circ$ instr $_{p}$ is the identity.

Tests of the form $1 \rightarrow n \cdot 1$, including scalars for $n=2$, and the true and false predicates $X \rightarrow 1+1$, are side-effect-free, simply because the composite $1 \rightarrow n \cdot 1 \rightarrow 1$ is necessarily the identity. Also, $p^{\perp}$ has the same side-effect as $p$, by the (Injection) property in Assumption 2, The side-effect associated with an effect $e \in[0,1]_{A}$ in a $C^{*}$-algebra $A$ is the map $A \rightarrow A$ given by $a \mapsto \sqrt{e} \cdot a \cdot \sqrt{e}+\sqrt{1-e} \cdot a \cdot \sqrt{1-e}$. If $A$ is commutative, this map is the identity. One of the main contributions of this section is establishing a connection between sideeffect-freeness and commutativity in $C^{*}$-algebras. We recall that commutative $C^{*}$-algebra are not 'quantum' but 'probabilistic' models of computation. Formulated differently, having side-effects of predicates/tests is a characteristic feature of the quantum world. Indeed, an observation at the quantum level may change (disturb) the state of the system that is being observed.

But first we show that there are no side-effects in classical and probabilistic logic.

Proposition 8.2. In the categories Sets, $\mathcal{K} \ell(\mathcal{D})$ and $\mathcal{K} \ell(\mathcal{G})$ all tests are side-effect-free. In those cases, there is a bijective correspondence:

$$
\frac{n \text {-tests } X \stackrel{p}{\longrightarrow} n \cdot 1}{\text { maps } X \underset{f}{\longrightarrow} n \cdot X \text { with } \nabla \circ f=\mathrm{id}}
$$

Downwards, the map $f$ corresponding to test $p$ is the instrument $f=$ instr $_{p}$ described in Example 7.2. Upwards, the test $p$ is obtained from $f$ as in Assumption (2) (4), namely as $p=(n \cdot !) \circ f$. 
Proof. The properties in Assumption 2 imply the bijective correspondence, once we have proved that tests are side-effects-free. In the set-theoretic case it is easy to see that the instrument (7.1) satisfies $\nabla \circ$ instr $_{p}=\mathrm{id}$. We shall skip $\mathcal{K} \ell(\mathcal{D})$ and do the calculation in $\mathcal{K} \ell(\mathcal{G})$. We must show that the instrument instr $p: X \rightarrow \mathcal{G}(n \cdot X)$ from (7.3), for a test $p: X \rightarrow \mathcal{G}(n)$, satisfies $\nabla \odot$ instr $_{p}=\mathrm{id}$, with Kleisli composition in $\mathcal{K} \ell(\mathcal{G})$ written as $\odot$. Well, for $x \in X$ and $M \in \Sigma_{X}$,

$$
\begin{aligned}
\left(\nabla \odot \operatorname{instr}_{p}\right)(x)(M) & =\nabla_{*}\left(\operatorname{instr}_{p}(x)\right)(M) \quad \text { where }(-)_{*} \text { is Kleisli extension } \\
& =\int \nabla(-)(M) \mathrm{d} \operatorname{instr}_{p}(x) \quad \text { see (A.3) } \\
& =\int \mathbf{1}_{\kappa_{1} M \cup \cdots \cup \kappa_{n} M \mathrm{~d} \operatorname{instr}_{p}(x)} \\
& =\operatorname{instr}_{p}(x)\left(\kappa_{1} M \cup \cdots \cup \kappa_{n} M\right) \\
& =\operatorname{instr}_{p}(x)\left(\kappa_{1} M\right)+\cdots+\operatorname{instr}_{p}(x)\left(\kappa_{n} M\right) \\
& =p(x)(1) \cdot \mathbf{1}_{M}(x)+\cdots+p(x)(n) \cdot \mathbf{1}_{M}(x) \quad \text { see (7.3) } \\
& =(p(x)(1)+\cdots+p(x)(n)) \cdot \mathbf{1}_{M}(x) \\
& =\mathbf{1}_{M}(x) \\
& =\eta(x)(M) \\
& =\operatorname{id}(x)(M) .
\end{aligned}
$$

The following classical result in the theory of $C^{*}$-algebras will be used without proof.

Theorem 1 (Tomiyama [81]). Let $A, B$ be $C^{*}$-algebras with two maps $f, g$ in:

$$
A \underset{\downarrow_{A}, \mathrm{PU}}{B} \quad \text { i.e. } \quad\left\{\begin{array}{l}
g \text { preserves multiplication, involution, unit (MIU) } \\
f \text { preserves unit, positive elements (PU) } \\
f \circ g=\mathrm{id}
\end{array}\right.
$$

Then $f$ is a map of modules, in the sense that, for all $a \in A, b \in B$,

$$
a \cdot f(b)=f(g(a) \cdot b) \quad \text { and } \quad f(b) \cdot a=f(b \cdot g(a)) .
$$

This result is also used in [27]. We shall apply it here with the diagonal $\Delta=\langle\mathrm{id}, \ldots, \mathrm{id}\rangle: A \rightarrow$ $A^{n}$ for $g$. It clearly preserves multiplication. This map $f$ is sometimes called a conditional expectation.

The following is a key result in linking side-effect-freeness in $\left(\mathbf{C s t a r}_{\mathrm{PU}}\right)^{\text {op }}$ to commutativity. Recall that the center $\mathcal{Z}(A) \subseteq A$ of a $C^{*}$-algebra $A$ is the sub- $C^{*}$-algebra of elements that commute with everything: $\mathcal{Z}(A)=\{a \in A \mid \forall x \in A \cdot a \cdot x=x \cdot a\}$.

Lemma 8.3. Let $A$ be a $C^{*}$-algebra. There is a bijective correspondence between:

$$
\frac{n \text {-tests } e_{1}, \ldots, e_{n} \in[0,1]_{A} \cap \mathcal{Z}(A) \text { of effects } e_{i} \text { in the center }}{\text { positive unital maps } f: A^{n} \rightarrow A \text { with } f \circ \Delta=\mathrm{id}}
$$

Proof. For effects $\vec{e}=e_{1}, \ldots, e_{n} \in[0,1]_{A}$ in the center $\mathcal{Z}(A)$ with $e_{1}+\cdots+e_{n}=1$ one can define a linear map $f_{\vec{e}}: A^{n} \rightarrow A$ by:

$$
f_{\vec{e}}\left(a_{1}, \ldots, a_{n}\right)=\sum_{i} e_{i} \cdot a_{i} .
$$


We use that the effects $e_{i}$ are in the center in order to show that this function $f_{\vec{e}}$ is positive: if $a_{i}=x_{i}^{*} x_{i}$, then $f_{\vec{e}}\left(a_{1}, \ldots, a_{n}\right)$ is positive, being the sum of positive elements:

$$
\begin{array}{rlr}
f_{\vec{e}}\left(a_{1}, \ldots, a_{n}\right)=\sum_{i} e_{i} \cdot a_{i} & =\sum_{i} e_{i} \cdot x_{i}^{*} \cdot x_{i} & \\
& =\sum_{i} x_{i}^{*} \cdot e_{i} \cdot x_{i} & \text { since } e_{i} \text { is in the center } \\
& =\sum_{i} x_{i}^{*} \cdot\left(\sqrt{e_{i}}\right)^{*} \cdot \sqrt{e_{i}} \cdot x_{i} & \text { since } e_{i} \text { is positive } \\
& =\sum_{i}\left(\sqrt{e_{i}} \cdot x_{i}\right)^{*} \cdot\left(\sqrt{e_{i}} \cdot x_{i}\right) .
\end{array}
$$

Clearly,

$$
\left(f_{\vec{e}} \circ \Delta\right)(a)=f_{\vec{e}}(a, \ldots, a)=\sum_{i} e_{i} \cdot a=\left(\sum_{i} e_{i}\right) \cdot a=1 \cdot a=a .
$$

In particular, $f_{\vec{e}}$ is unital. The effects $e_{i}$ can be retrieved from $f_{\vec{e}}$ as $e_{i}=f_{\vec{e}}(|i\rangle)$, where $|i\rangle \in A^{n}$ is the sequence with $1 \in A$ at position $i$ and $0 \in A$ elsewhere.

Conversely, let $f: A^{n} \rightarrow A$ be a positive unital map with $f \circ \Delta=$ id. We obtain effects $e_{i}=f(|i\rangle) \in[0,1]_{A}$ in the standard way. And we get $f=f_{\vec{e}}$ via Tomiyama's Theorem [1;

$$
\begin{aligned}
f\left(a_{1}, \ldots, a_{n}\right)=f\left(\sum_{i}|i\rangle \cdot \Delta\left(a_{i}\right)\right)=\sum_{i} f\left(|i\rangle \cdot \Delta\left(a_{i}\right)\right) & =\sum_{i} f(|i\rangle) \cdot a_{i} \quad \text { see (8.2) } \\
& =\sum_{i} e_{i} \cdot a_{i} \\
& =f_{\vec{e}}\left(a_{1}, \ldots, a_{n}\right) .
\end{aligned}
$$

We still need to prove that the effects $e_{i}=f(|i\rangle)$ are in the center. Again, this follows from Tomiyama's Theorem:

$$
\begin{array}{rlr}
e_{i} \cdot x=f(|i\rangle) \cdot x & =f(|i\rangle \cdot \Delta(x)) & \text { see (8.2) } \\
& =f(\Delta(x) \cdot|i\rangle) \\
& =x \cdot f(|i\rangle) & \\
& =x \cdot e_{i} .
\end{array}
$$

Corollary 8.4. Let $\vec{e}=e_{1}, \ldots, e_{n} \in[0,1]_{A}$ be an n-test in a $C^{*}$-algebra A. Then:

$\vec{e}$ is side-effect-free $\Longleftrightarrow$ each effect $e_{i}$ is in the center $\mathcal{Z}(A)$.

Proof. First we observe that if $e \in[0,1]_{A} \cap \mathcal{Z}(A)$, then also $\sqrt{e} \in[0,1]_{A} \cap \mathcal{Z}(A)$. That the square root $\sqrt{e}$ is an effect is obvious. It is also in the center $\mathcal{Z}(A) \hookrightarrow A$ since this center is sub- $C^{*}$-algebra, so the construction of $\sqrt{e}$ for $e \in \mathcal{Z}(A)$ via the functional calculus takes place entirely within $\mathcal{Z}(A)$. Secondly we recall that the instrument instr $\operatorname{er}_{\vec{e}}: A^{n} \rightarrow A$ for the $n$-test $\vec{e}$ is given by $\operatorname{instr}_{\vec{e}}(\vec{a})=\sum_{i} \sqrt{e_{i}} \cdot a_{i} \cdot \sqrt{e_{i}}$, see (7.5).

For the implication $(\Longleftarrow)$ assume that the effects $e_{i}$ are in the center $\mathcal{Z}(A)$. Then so are the $\sqrt{e_{i}}$, and thus:

$$
\operatorname{instr}_{\vec{e}}(\vec{a})=\sum_{i} \sqrt{e_{i}} \cdot a_{i} \cdot \sqrt{e_{i}}=\sum_{i} \sqrt{e_{i}} \cdot \sqrt{e_{i}} \cdot a_{i}=\sum_{i} e_{i} \cdot a_{i}=f_{\vec{e}}(\vec{a}),
$$

where the function $f_{\vec{e}}$ is as defined in (8.3). This yields $\operatorname{instr}_{\vec{e}} \circ \Delta=f_{\vec{e}} \circ \Delta=\mathrm{id}$, as shown in the proof of Lemma 8.3, so that the $n$-test $\vec{e}$ is side-effect-free.

For $(\Longrightarrow)$, if $\operatorname{instr}_{\vec{e}} \circ \Delta=$ id, then by Lemma 8.3 instr $_{\vec{e}}=f_{\vec{d}}$ where $d_{i} \in[0,1]_{A} \cap \mathcal{Z}(A)$ is defined as: $d_{i}=\operatorname{instr}_{\vec{e}}(|i\rangle)=\sqrt{e_{i}} \cdot 1 \cdot \sqrt{e_{i}}=e_{i}$.

There is another conclusion we can draw at this stage: the instruments $\operatorname{instr}_{\vec{e}}$ for $C^{*}$ algebras defined in (7.5) satisfy the 'side-effect' property from Assumption 2 (44). This was postponed at the end of Example 7.2 . 
Corollary 8.5. The category $\left(\text { Cstar }_{P U}\right)^{o p}$ satisfies the measurement Assumption Q: in particular, the Side-effect-free property holds.

Proof. Given a positive unital map $f: A^{n} \rightarrow A$ with $f \circ \Delta=$ id, we obtain an $n$-test $q=f \circ !^{n}: \mathbb{C}^{n} \rightarrow A$ corresponding to effects $e_{i}=q(|i\rangle)=f(|i\rangle)$ in the center. But then $f=$ instr $_{\vec{e}}$ as shown in the previous proof, and thus instr $q=$ instr $_{\vec{e}}=f$.

For our next result it is useful to make the following observation explicit.

Lemma 8.6. Let $a \in A$ be an element of a $C^{*}$-algebra $A$. Then:

$$
\forall x \in A \cdot a \cdot x=x \cdot a \Longleftrightarrow \forall e \in[0,1]_{A} \cdot a \cdot e=e \cdot a .
$$

In words: a commutes with all elements $x \in A$ if and only if it commutes with all effects $e \in[0,1]_{A}$.

Proof. The direction $(\Rightarrow)$ is obvious, so we concentrate on $(\Leftarrow)$. A standard result in the theory of $C^{*}$-algebras says that we can write each $x \in A$ as linear combination $x=$ $x_{1}-x_{2}+i x_{3}-i x_{4}$ of positive elements $x_{i} \geq 0$. By suitable scaling we can write $x=$ $\frac{1}{r}\left(r x_{1}-r x_{2}+i r x_{3}-i r x_{4}\right)$, where $r x_{i} \leq 1$ is an effect, for $r>0$. By assumption, each $r x_{i} \in[0,1]_{A}$ commutes with $a$. But then $x$ itself also commutes with $a$ since:

$$
\begin{aligned}
a \cdot x & =a \cdot \frac{1}{r}\left(r x_{1}-r x_{2}+i r x_{3}-i r x_{4}\right) \\
& \left.=\frac{1}{r}\left(a \cdot r x_{1}-a \cdot r x_{2}+i a \cdot r x_{3}\right)-i a \cdot r x_{4}\right) \\
& =\frac{1}{r}\left(r x_{1} \cdot a-r x_{2} \cdot a+i r x_{3} \cdot a-i r x_{4} \cdot a\right) \\
& =\frac{1}{r}\left(r x_{1}-r x_{2}+i r x_{3}-i r x_{4}\right) \cdot a \\
& =x \cdot a .
\end{aligned}
$$

Corollary 8.7. $A C^{*}$-algebra is commutative if and only if all its tests are side-effect-free.

Proof. If a $C^{*}$-algebra $A$ is commutative, then $\mathcal{Z}(A)=A$, so in particular each effect $e \in[0,1]_{A}$ is in the center, so that each test $\vec{e}$ is side-effect-free by Corollary 8.4 . Conversely, if each test is side-effect-free, then each effect $e \in[0,1]_{A}$, considered as predicate (i.e. 2-test), is in the center. An arbitrary element $a \in A$ thus commutes with each effect $e$. But this $a$ then commutes with all other elements, by Lemma 8.6. This makes $A$ commutative.

In [19] so-called von Neumann projective measurements on Hilbert spaces are described as (Eilenberg-Moore) coalgebras of a comonad. This result can be adapted to the current setting, but requires side-effect-freeness.

Definition 8.8. A von Neumann $n$-test is an $n$-test $e_{1}, \ldots, e_{n}$ of effects in a $C^{*}$-algebra satisfying, for each $i, j$,

$$
e_{i} \cdot e_{j}= \begin{cases}e_{i} & \text { if } i=j \\ 0 & \text { otherwise }\end{cases}
$$

Such test will be called a central von Neumann test if each $e_{i}$ is in the center.

Notice that each effect $e_{i}$ in a von Neumann test is a projection: $e_{i}^{2}=e_{i}$. As a result, $\sqrt{e_{i}}=e_{i}$

In [41] it is observed that, in general, for a category with coproducts + , for each $n>0$ taking $n$-fold copowers $X \mapsto n \cdot X=X+\cdots+X$ is a comonad, with counit $\varepsilon$ and comultiplication $\delta$ :

$$
n \cdot X \stackrel{\varepsilon=\nabla=[\mathrm{id}, \ldots, \mathrm{id}]}{\longrightarrow} X \quad n \cdot X \stackrel{\delta=\kappa_{1}+\cdots+\kappa_{n}}{\longrightarrow} n \cdot(n \cdot X)
$$


We shall be using this comonad on the opposite category $\left(\mathbf{C s t a r}_{\mathrm{PU}}\right)^{\mathrm{op}}$. It forms a monad $(-)^{n}$ on Cstar $_{\mathrm{PU}}$, with unit $\Delta$ and multiplication $\pi_{1}+\cdots+\pi_{n}$. We then have the following result, describing the instruments of central von Neumann tests as coalgebras of a comonad.

Theorem 2. For a $C^{*}$-algebra $A$ there is a bijective correspondence between:

$$
\frac{\text { central von Neumann } n \text {-tests } e_{1}, \ldots, e_{n} \in[0,1]_{A}}{\text { coalgebras } f: A \rightarrow n \cdot A \text { in }\left(\text { Cstar }_{\mathrm{PU}}\right)^{\text {op }}}
$$

This is a restriction of the bijective correspondence in Lemma 8.3 ,

Proof. Given a central von Neumann test $\vec{e}=e_{1}, \ldots, e_{n}$ we already know that the corresponding function $f_{\vec{e}}$ from (8.3) satisfies $f_{\vec{e}} \circ \Delta=\mathrm{id}$. We show that the orthogonal projection requirement (8.4) is equivalent to the (co)algebra equation:

$$
f_{\vec{e}} \circ\left(f_{\vec{e}}\right)^{n}=f_{\vec{e}} \circ\left(\pi_{1} \oplus \cdots \oplus \pi_{n}\right):\left(A^{n}\right)^{n} \longrightarrow A .
$$

This equivalence is the essence of [19, Thm. 16.6]. Here it occurs in $C^{*}$-algebraic terms.

So assume $\vec{e}$ is a (central) von Neumann test. Then for $n$ sequences $\overrightarrow{a_{i}}=\left(a_{i 1}, \ldots, a_{i n}\right) \in$ $A^{n}$ we can prove (8.5):

$$
\begin{array}{rlr}
\left(f_{\vec{e}} \circ\left(f_{\vec{e}}\right)^{n}\right)\left(\overrightarrow{a_{1}}, \ldots, \overrightarrow{a_{n}}\right) & =f_{\vec{e}}\left(f_{\vec{e}}\left(\overrightarrow{a_{1}}\right), \ldots, f_{\vec{e}}\left(\overrightarrow{a_{n}}\right)\right) & \\
& =\sum_{i} e_{i} \cdot\left(\sum_{j} e_{j} \cdot a_{i j}\right) & \\
& =\sum_{i, j} e_{i} \cdot e_{j} \cdot a_{i j} & \text { by (8.4) } \\
& =\sum_{i} e_{i} \cdot a_{i i} \\
& =f_{\vec{e}}\left(a_{11}, \ldots, a_{n n}\right) \\
& =f_{\vec{e}}\left(\pi_{1}\left(\overrightarrow{a_{1}}\right), \ldots, \pi_{n}\left(\overrightarrow{a_{n}}\right)\right) \\
& =\left(f_{\vec{e}} \circ\left(\pi_{1} \oplus \cdots \oplus \pi_{n}\right)\right)\left(\overrightarrow{a_{1}}, \ldots, \overrightarrow{a_{n}}\right) .
\end{array}
$$

In the reverse direction, assuming (8.5) we prove (8.4). Take the sequence of sequences:

$$
s=(\overrightarrow{0}, \ldots, \overrightarrow{0},|j\rangle, \overrightarrow{0}, \ldots, \overrightarrow{0}) \in\left(A^{n}\right)^{n}
$$

with $|j\rangle \in A^{n}$ at the $i$-th position. Then:

$$
\begin{aligned}
& e_{i} \cdot e_{j}=e_{i} \cdot f_{\vec{e}}(|j\rangle) \\
& =f_{\vec{e}}\left(0, \ldots, 0, f_{\vec{e}}(|j\rangle), 0, \ldots, 0\right) \\
& =f_{\vec{e}}\left(f_{\vec{e}}(\overrightarrow{0}), \ldots, f_{\vec{e}}(\overrightarrow{0}), f_{\vec{e}}(|j\rangle), f_{\vec{e}}(\overrightarrow{0}), \ldots, f_{\vec{e}}(\overrightarrow{0})\right) \\
& =\left(f_{\vec{e}} \circ\left(f_{\vec{e}}\right)^{n}\right)(s) \\
& =\left(f_{\vec{e}} \circ\left(\pi_{1} \oplus \cdots \oplus \pi_{n}\right)\right)(s) \quad \text { by (8.5) } \\
& = \begin{cases}f_{\vec{e}}(0, \ldots, 0,1,0 \ldots, 0) & \text { if } i=j \\
f_{\vec{e}}(0, \ldots, 0,0,0 \ldots, 0) & \text { otherwise }\end{cases} \\
& = \begin{cases}e_{i} & \text { if } i=j \\
0 & \text { otherwise. }\end{cases}
\end{aligned}
$$

We conclude this section by introducing 'pure' maps, which commute appropriately with instruments. 
Definition 8.9. In an effectus with chosen instruments, as in Assumption 2, we call a map $f: X \rightarrow Y$ pure if for each $n$-test $q: Y \rightarrow n \cdot 1$ the following diagram commutes.

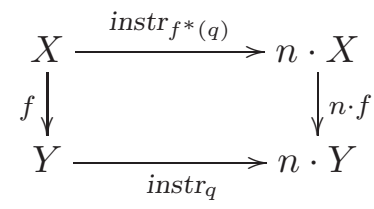

This makes $f$ a map of coalgebras, for the functor $n \cdot(-)$.

We should point out that 'purity' depends on the instruments that are used. The next result shows that by restricting ourselves to pure maps we still have a category with coproducts.

Lemma 8.10. The pure maps form a subcategory: the identity map is pure, and if $f, g$ are composable pure maps, then $g \circ f$ is pure too. Also, the coprojections are pure, and if $f_{i}: X_{i} \rightarrow Y$ is pure for $i=1,2$, then so is the cotuple $\left[f_{1}, f_{2}\right]$.

Proof. Closure under composition is obvious. We shall prove that coprojections are pure. Closure under cotuples is done similarly, using Assumption 2 (2). Consider a coprojection $\kappa_{1}: X \rightarrow X+Y$ and an $n$-test $q=\left[q_{1}, q_{2}\right]: X+Y \rightarrow n \cdot 1$ on its codomain. Then:

$$
\begin{aligned}
\text { instr }_{q} \circ \kappa_{1} & =\left[\kappa_{1}+\kappa_{1}, \ldots, \kappa_{n}+\kappa_{n}\right]^{-1} \circ\left(\text { instr }_{q_{1}}+\text { instr }_{q_{2}}\right) \circ \kappa_{1} \quad \text { by Assumption 2 (2) } \\
& =\left[n \cdot \kappa_{1}, n \cdot \kappa_{2}\right] \circ \kappa_{1} \circ \text { instr }_{q_{1}} \\
& =\left(n \cdot \kappa_{1}\right) \circ \text { instr }_{\kappa_{1}^{*}(q)} .
\end{aligned}
$$

The next result describes pure maps in several examples. Note that we only give sufficient conditions, and no characterisations of pure maps in these cases.

Proposition 8.11. In our standard examples we have the following situation.

(1) In Sets all maps are pure.

(2) In $\mathcal{K} \ell(\mathcal{D})$ all maps in the image of the inclusion $J:$ Sets $\rightarrow \mathcal{K} \ell(\mathcal{D})$ are pure.

(3) Similarly, in $\mathcal{K} \ell(\mathcal{G})$ all maps in the image of the inclusion $J:$ Meas $\rightarrow \mathcal{K} \ell(\mathcal{G})$ are pure.

(4) In $_{\text {Cstar }_{P U}}$ all MIU-maps are pure, i.e. all maps in the image of $\boldsymbol{C s t a r}_{M I U} \rightarrow \boldsymbol{C s t a r}_{P U}$.

Proof. For $f: X \rightarrow Y$ in Sets and $q: Y \rightarrow n$ we have:

$$
\begin{aligned}
\left(n \cdot f \circ \operatorname{instr}_{q \circ f}\right)(x)=\kappa_{i} y & \Longleftrightarrow \operatorname{instr}_{q \circ f}(x)=\kappa_{i} x \text { and }(n \cdot f)\left(\kappa_{i} x\right)=\kappa_{i} y \\
& \Longleftrightarrow q(f(x))=i \text { and } f(x)=y \\
& \Longleftrightarrow \operatorname{instr}_{q}(f(x))=\kappa_{i} f(x) \text { and } f(x)=y \\
& \left.\Longleftrightarrow \operatorname{instr}_{q} \circ f\right)(x)=\kappa_{i} y
\end{aligned}
$$

In $\mathcal{K} \ell(\mathcal{D})$ a map $f: X \rightarrow Y$ of the form $f=\eta \circ g$ is pure, for a function $g: X \rightarrow Y$ in Sets, where $\eta$ is the unit $Y \rightarrow \mathcal{D}(Y)$ given by $\eta(y)=1|y\rangle$. Then $n \cdot f: n \cdot X \rightarrow \mathcal{D}(n \cdot Y)$ is given by $(n \cdot f)\left(\kappa_{i} x\right)=1\left|\kappa_{i} g(x)\right\rangle$. And for a predicate $q: Y \rightarrow \mathcal{D}(n)$ we have $q \odot f=q \circ g$. 
Hence:

$$
\begin{aligned}
\left(n \cdot f \odot \operatorname{instr}_{q \odot f}\right)(x) & \left.=(n \cdot f)_{*}\left(\sum_{i} q(g(x))(i)\left|\kappa_{i} x\right\rangle\right) \quad \text { with }(-)_{*} \text { from (1.2. }\right) \\
& =\sum_{i} q(g(x))(i)\left|\kappa_{i} g(x)\right\rangle \\
& =\operatorname{instr}_{q}(g(x)) \\
& =\left(\operatorname{instr}_{q} \odot f\right)(x) .
\end{aligned}
$$

The Giry monad $\mathcal{G}$ is handled similarly. Finally, in the case of $C^{*}$-algebras, a MIU-map $f: B \rightarrow A$ preserves not only multiplication but also square roots. Hence, for an $n$-test $\mathbb{C}^{n} \rightarrow B$, corresponding to effects $e_{i}=q(|i\rangle) \in B$ with $\emptyset_{i} e_{i}=1$, we have:

$$
\begin{aligned}
\left(\operatorname{instr}_{f \circ \vec{e}} \circ f^{n}\right)\left(b_{1}, \ldots, b_{n}\right) & =\operatorname{instr}_{f\left(\vec{e}_{i}\right)}\left(f\left(b_{1}\right), \ldots, f\left(b_{n}\right)\right) \\
& =\sum_{i} \sqrt{f\left(e_{i}\right)} \cdot f\left(b_{i}\right) \cdot \sqrt{f\left(e_{i}\right)} \\
& =\sum_{i} f\left(\sqrt{e_{i}}\right) \cdot f\left(b_{i}\right) \cdot f\left(\sqrt{e_{i}}\right) \\
& =\sum_{i} f\left(\sqrt{e_{i}} \cdot b_{i} \cdot \sqrt{e_{i}}\right) \\
& =f\left(\sum_{i} \sqrt{e_{i}} \cdot b_{i} \cdot \sqrt{e_{i}}\right) \\
& =\left(f \circ \text { instr }_{\vec{e}}\right)\left(b_{1}, \ldots, b_{n}\right) .
\end{aligned}
$$

Below we give an illustration of a map that is not of the form $\eta \circ f$ in $\mathcal{K} \ell(\mathcal{D})$ and which is not pure. Thus the condition in point (2) is necessary. It is not clear if the MIU condition in point 4 is also necessary. For states, it is known that a MIU-map $\omega: A \rightarrow \mathbb{C}$ is pure in the sense that each linear map $f: A \rightarrow \mathbb{C}$ for which both $f$ and $\omega-f$ are positive is of the form $f=r \cdot \omega$ for some $r \in[0,1]$. This latter formulation is equivalent to $\omega$ being an extreme point in the convex set of PU maps $A \rightarrow \mathbb{C}$.

Example 8.12. Let state $\omega: 1 \rightarrow \mathcal{D}(\{a, b\})$ in $\mathcal{K} \ell(\mathcal{D})$ be given by: $\omega=\frac{1}{4}|a\rangle+\frac{3}{4}|b\rangle$ and predicate $q:\{a, b\} \rightarrow[0,1]$ by $q(a)=\frac{2}{5}, q(b)=\frac{1}{3}$. The instrument instr $q:\{a, b\} \rightarrow$ $\mathcal{D}(\{a, b\}+\{a, b\})$ is:

$$
\operatorname{instr}_{q}(a)=\frac{2}{5}\left|\kappa_{1} a\right\rangle+\frac{3}{5}\left|\kappa_{2} a\right\rangle \quad \operatorname{instr}_{q}(b)=\frac{1}{3}\left|\kappa_{1} b\right\rangle+\frac{2}{3}\left|\kappa_{2} b\right\rangle .
$$

Thus $\operatorname{instr}_{q} \odot \omega: 1 \rightarrow \mathcal{D}(\{a, b\}+\{a, b\})$ is:

$$
\operatorname{instr}_{q} \odot \omega=\frac{1}{10}\left|\kappa_{1} a\right\rangle+\frac{3}{20}\left|\kappa_{2} a\right\rangle+\frac{1}{4}\left|\kappa_{1} b\right\rangle+\frac{1}{2}\left|\kappa_{2} b\right\rangle .
$$

On the other hand, $\omega^{*}(q): 1 \rightarrow[0,1]$ is given by $\omega^{*}(q)=\frac{1}{4} \cdot \frac{2}{5}+\frac{3}{4} \cdot \frac{1}{3}=\frac{1}{10}+\frac{1}{4}=\frac{7}{20}$. The associated instrument $\operatorname{instr}_{\omega^{*}(q)}: 1 \rightarrow \mathcal{D}(1+1)$ is $\operatorname{instr}_{\omega^{*}(q)}=\frac{7}{20}|0\rangle+\frac{13}{20}|1\rangle$. Then $(\omega+\omega) \odot \operatorname{instr}_{\omega^{*}(q)}: 1 \rightarrow \mathcal{D}(\{a, b\}+\{a, b\})$ is the distribution

$$
(\omega+\omega) \odot \operatorname{instr}_{\omega^{*}(q)}=\frac{7}{80}\left|\kappa_{1} a\right\rangle+\frac{21}{80}\left|\kappa_{1} b\right\rangle+\frac{13}{80}\left|\kappa_{2} a\right\rangle+\frac{39}{80}\left|\kappa_{2} b\right\rangle .
$$

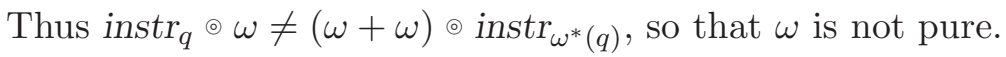

Finally, we observe that for a pure map $g$ one has the following pre-composition property for test maps, as defined in (7.6)

$$
\left(p ?\left[f_{1}, \ldots, f_{n}\right]\right) \circ g=\left(g^{*}(p)\right) ?\left[f_{1} \circ g, \ldots, f_{n} \circ g\right] .
$$




\section{TEST OPERATORS IN DYNAMIC LOGIC}

At this stage both our assumptions will be used to define test operators on predicates. It will provide the basis for a dynamic logic whose semantic basis will be investigated in this section. We do not provide a proper logic with deduction rules, but only describe what holds in categorical models.

Definition 9.1. Let $\mathbf{B}$ be an effectus with a choice of instruments instr ${ }_{p}$ as in Assumptions 1 and 2. For two predicates $p, q: X \rightarrow 1+1$ on the same object $X \in \mathbf{B}$ we define two new "test" predicates on $X$, namely:

$$
\begin{aligned}
& \langle p ?\rangle(q)=\left(\operatorname{instr}_{p}\right)^{*}(\text { FstAnd }(q))=[q, 0] \circ \operatorname{instr}_{p}: X \longrightarrow X+X \longrightarrow 1+1 \\
& {[p ?](q)=\left(\text { instr }_{p}\right)^{*}(\text { FstThen }(q))=[q, 1] \circ \operatorname{instr}_{p}: X \longrightarrow X+X \longrightarrow 1+1 .}
\end{aligned}
$$

We call $\mathbf{B}$ a commutative model if $\langle p ?\rangle(q)=\langle q ?\rangle(p)$ for all predicates $p, q$. Also, we call a predicate $p$ a projection if $\langle p ?\rangle(p)=p$. Such projections are sometimes called 'sharp' predicates, and, in contrast, effects are then the 'unsharp' ones.

We pronounce $\langle p ?\rangle(q)$ as "test $p$, and then $q$ ", and $[p ?](q)$ as "test $p$, then $q$ ". These $\langle p ?\rangle(q)$ and $[p ?](q)$ are test operators, like in dynamic logic, see [34. Using the test map notation from (7.7) we may also describe these test operators as:

$$
\langle p ?\rangle(q)=\text { if } \begin{aligned}
& \mid \begin{array}{l}
p \rightarrow q \\
p^{\perp} \rightarrow 0
\end{array} \\
& \text { fi }
\end{aligned}
$$

These notations emphasise the difference between $p$ and $q$ in these test operators, since $p$ is used for an action, namely measurement, via the associated instrument instr ${ }_{p}$, after which $q$ is evaluated. In dynamic logic the test $p$ ? has no side-effect, that is, it does not change any state. But the current setting such a test $p$ ? may have a side-effect, see explicitly in Corollary 9.5 below. Therefore, commutativity does not hold in general, as the $C^{*}$-algebra example below demonstrates. We now review the meaning of $\langle p ?\rangle(q)$ and $[p$ ?] $(q)$ in our running examples. We will see that familiar logical operations emerge from the above general definitions.

Examples 9.2. In the category Sets, for predicates $p, q: X \rightarrow 2$ we have:

$$
\begin{aligned}
& \langle p ?\rangle(q)(x)=[q, 0]\left(\operatorname{instr}_{p}(x)\right)=\left\{\begin{array}{ll}
q(x) & \text { if } p(x)=1 \\
0 & \text { otherwise }
\end{array}\right\}=p(x) \cdot q(x) \\
& {[p ?](q)(x)=[q, 1]\left(\operatorname{instr}_{p}(x)\right)=\left\{\begin{array}{ll}
q(x) & \text { if } p(x)=1 \\
1 & \text { otherwise }
\end{array}\right\}=p(x) \cdot q(x)+(1-p(x))}
\end{aligned}
$$

These formulas describe ordinary conjunction and implication in terms of characteristic functions. Since $p(x) \in\{0,1\}$ we have $p(x) \cdot p(x)=p(x)$, so that each predicate is a projection. This set-theoretic model is clearly commutative: $\langle p ?\rangle(q)=\langle p ?\rangle(q)$ holds since multiplication on $\{0,1\}$ is commutative.

In the Kleisli category $\mathcal{K} \ell(\mathcal{D})$ of the distribution monad we get the same formulas, but this time interpreted not in the set of Booleans $\{0,1\}$ but in the unit interval $[0,1]$. For $p, q: X \rightarrow[0,1]$ we elaborate $\langle p ?\rangle(q)$ as function $X \rightarrow \mathcal{D}(2)$. We first write the cotuple 
$[q, 0]: X+X \rightarrow \mathcal{D}(2)$ as $[q, 0]\left(\kappa_{1} x\right)=q(x)|1\rangle+(1-q(x))|0\rangle$ and $[q, 0]\left(\kappa_{2} x\right)=1|0\rangle$. Then:

$$
\begin{aligned}
\langle p ?\rangle(q)(x) & =\left([q, 0] \odot \operatorname{instr}_{p}\right)(x) \\
& =[q, 0]_{*}\left(p(x)\left|\kappa_{1} x\right\rangle+(1-p(x))\left|\kappa_{2} x\right\rangle\right) \quad \text { with }(-)_{*} \text { from (A.2) } \\
& =p(x) \cdot q(x)|1\rangle+p(x) \cdot(1-q(x))|0\rangle+(1-p(x)) \cdot 1|0\rangle \\
& =p(x) \cdot q(x)|1\rangle+(1-p(x) \cdot q(x))|0\rangle .
\end{aligned}
$$

Thus, as fuzzy predicate $\langle p ?\rangle(q): X \rightarrow[0,1]$ we can write $\langle p ?\rangle(q)(x)=p(x) \cdot q(x)$ like in the set-theoretic case. Hence, $\mathcal{K} \ell(\mathcal{D})$ is a commutative model.

The projections $p$ are the predicates with $p(x)^{2}=p(x)$, so that $p(x) \in\{0,1\}$, and are thus the Boolean predicates. One can check that the then-test is given by $[p ?](q)(x)=$ $p(x) \cdot q(x)+(1-p(x))$. In probability theory the latter formula for the then-test is called the Reichenbach implication, see [69].

Precisely the same formulas hold for predicates in the Kleisli category $\mathcal{K} \ell(\mathcal{G})$ of the Giry monad, for measurable maps $X \rightarrow \mathcal{G}(2) \cong[0,1]$ where $X$ is a measurable space, see also [40, Prop. 13].

In the (opposite of the) category of commutative rings, assume we have two predicates (idempotents) $r, s \in R$, considered as ring homomorphisms $f_{r}, f_{s}: \mathbb{Z}^{2} \rightarrow R$, like in Example 3.2 (15). The instrument instr $r_{r}: R^{2} \rightarrow R$ associated with $r$ is given by $\operatorname{instr}_{r}(x, y)=$ $r \cdot x+(1-r) \cdot y$. The element $\langle r ?\rangle(s) \in R$ is defined as $\langle r ?\rangle(s)=f(1,0)$ for the function $f: \mathbb{Z}^{2} \rightarrow R$ given by:

$$
\begin{aligned}
f(n, m)=\left(\operatorname{instr}_{r} \circ\left\langle f_{s}, f_{0}\right\rangle\right)(n, m) & =\operatorname{instr}_{r}(n \cdot s+m \cdot(1-s), m \cdot 1) \\
& =r \cdot(n \cdot s+m \cdot(1-s))+(1-r) \cdot(m \cdot 1) \\
& =n \cdot(r \cdot s)+m \cdot(1-r \cdot s) .
\end{aligned}
$$

Hence $\langle r ?\rangle(s)=r \cdot s$. Similarly, $[r ?](s)=r \cdot s+1-r$. In a same manner one obtains, for complementable elements $x, y$ in a distributive lattice, $\langle x ?\rangle(y)=x \wedge y$ and $[x ?](y)=$ $(x \wedge y) \vee x^{\perp}=y \vee x^{\perp}$.

In a $C^{*}$-algebra $A$, for effects $e, d \in[0,1]_{A}$, we can compute $\langle e ?\rangle(d)$ via the map $f: \mathbb{C}^{2} \rightarrow$ $A$ given by:

$$
\begin{aligned}
f(z, w) & =\operatorname{instr}_{e}(\langle d, 0\rangle(z, w)) \\
& =\operatorname{instr}_{e}(z \cdot d+w \cdot(1-d), w \cdot 1) \\
& =\sqrt{e} \cdot(z \cdot d+w \cdot(1-d)) \cdot \sqrt{e}+\sqrt{1-e} \cdot(w \cdot 1) \cdot \sqrt{1-e} \\
& =z \cdot \sqrt{e} \cdot d \cdot \sqrt{e}+w \cdot \sqrt{e} \cdot(1-d) \cdot \sqrt{e}+w \cdot(1-e) .
\end{aligned}
$$

We get the corresponding effect by taking $z=1, w=0$. Then:

$$
\langle e ?\rangle(d)=f(1,0)=\sqrt{e} \cdot d \cdot \sqrt{e} \quad \text { and similarly } \quad[e ?](d)=\sqrt{e} \cdot d \cdot \sqrt{e}+1-e .
$$

This formula for $\langle e ?\rangle(d)$ is precisely the one that is used in [31, 32], for effects on a Hilbert space, as instance of the notion of 'sequential product' on effect algebras.

By definition, in the current context, a projection is an effect $e \in[0,1]_{A}$ satisfying $e=\langle e ?\rangle(e)$. This means $e=\sqrt{e} \cdot e \cdot \sqrt{e}=\sqrt{e} \cdot \sqrt{e} \cdot \sqrt{e} \cdot \sqrt{e}=e \cdot e=e^{2}$. Hence projections are idempotent effects. Equivalently, following the more common description, an idempotent is an element $a \in A$ satisfying $a^{2}=a=a^{*}$. Notice that for such a projection $a$ one has 
$\sqrt{a}=a$ and thus $\langle a ?\rangle(\langle a ?\rangle(e))=\langle a ?\rangle(e)$ for each effect $e$, since:

$$
\langle a ?\rangle(\langle a ?\rangle(e))=\sqrt{a} \cdot(\sqrt{a} \cdot e \cdot \sqrt{a}) \cdot \sqrt{a}=a \cdot e \cdot a=\sqrt{a} \cdot e \cdot \sqrt{a}=\langle a ?\rangle(e) .
$$

Hence a double andthen-test $\langle a ?\rangle(-)$ with a projection $a$ is the same as a single test. This holds not only for andthen-tests, but also for then-tests $[a$ ? $](-)$, by the De Morgan property from Lemma $9.3(7)$ below.

Next we collect some basic result about our test operators.

Lemma 9.3. The andthen-test and then-test operators $\langle-?\rangle(-)$ and $[-?](-)$ in a category

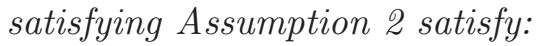

(1) $\langle 1 ?\rangle(p)=p=\langle p ?\rangle(1)$;

(2) $\langle 0 ?\rangle(p)=0=\langle p ?\rangle(0)$;

(3) $\langle p ?\rangle\left(q_{1} \otimes q_{2}\right)=\langle p ?\rangle\left(q_{1}\right) \otimes\langle p ?\rangle\left(q_{2}\right)$;

(4) $\langle p ?\rangle(s \bullet q)=s \bullet\langle p ?\rangle(q)$, for a scalar $s$;

(5) $\langle p ?\rangle(q) \leq p$;

(6) $q_{1} \leq q_{2}$ implies $\langle p ?\rangle\left(q_{1}\right) \leq\langle p ?\rangle\left(q_{2}\right)$;

(7) $[p ?](q)=\langle p ?\rangle\left(q^{\perp}\right)^{\perp}$;

(8) $\langle p ?\rangle(q) \otimes p^{\perp}=[p ?](q)$, and so $\langle p ?\rangle(q) \leq[p ?](q)$;

(9) $\left\langle\left[p_{1}, p_{2}\right] ?\right\rangle(q)=\left[\left\langle p_{1} ?\right\rangle\left(q \circ \kappa_{1}\right),\left\langle p_{1} ?\right\rangle\left(q \circ \kappa_{2}\right)\right]$;

(10) $f^{*}(\langle p ?\rangle(q))=\left\langle f^{*}(p) ?\right\rangle\left(f^{*}(q)\right)$ if $f$ is a pure map.

Via point (7) (and the other points) one obtains various properties of $[p ?](-)$. For instance,

$$
[p ?](0) \stackrel{\square(7)]}{=}\langle p ?\rangle(1)^{\perp \stackrel{(1)}{=}} p^{\perp} \quad[p ?](1) \stackrel{(7)]}{=}\langle p ?\rangle(0)^{\perp \stackrel{(2)}{=}} 0^{\perp}=1 .
$$

Proof. We describe some relevant steps, using the properties of the FstAnd operator from Lemma 6.3.

(1) Obviously, $\langle 1 ?\rangle(p)=\left(\operatorname{instr}_{1}\right)^{*}(\operatorname{FstAnd}(p))=\operatorname{FstAnd}(p) \circ \kappa_{1}=[p, 0] \circ \kappa_{1}=p$. And:

$$
\langle p ?\rangle(1)=\text { FstAnd(1) } \circ \operatorname{instr}_{p}=\left[\kappa_{1} \circ !, \kappa_{2} \circ !\right] \circ \operatorname{instr}_{p}=(!+!) \circ \operatorname{instr}_{p}=p .
$$

(2) Like in the previous point, $\langle 0 ?\rangle(p)=\left(\operatorname{instr}_{0}\right)^{*}([p, 0])=[p, 0] \circ \kappa_{2}=0$. And for the equation $\langle p ?\rangle(0)=0$, we use Lemma 6.3 (3) in:

$$
\langle p ?\rangle(0)=\left(\operatorname{instr}_{p}\right)^{*}(\text { FstAnd }(0))=\left(\text { instr }_{p}\right)^{*}(0)=0 .
$$

(3) Lemma 6.3 (4) says that FstAnd preserves $\emptyset$, and thus:

$$
\begin{aligned}
& \langle p ?\rangle\left(q_{1} \otimes q_{2}\right)=\left(\text { instr }_{p}\right) *\left(\text { FstAnd }\left(q_{1} \otimes q_{2}\right)\right) \\
& =\left(\operatorname{instr}_{p}\right)^{*}\left(\operatorname{FstAnd}\left(q_{1}\right) \otimes \operatorname{FstAnd}\left(q_{2}\right)\right) \\
& =\left(\operatorname{instr}_{p}\right)^{*}\left(F_{\text {stAnd }}\left(q_{1}\right)\right) \otimes\left(\text { instr }_{p}\right)^{*}\left(F_{\text {stAnd }}\left(q_{2}\right)\right) \\
& =\langle p ?\rangle\left(q_{1}\right) \otimes\langle p ?\rangle\left(q_{2}\right) \text {. }
\end{aligned}
$$

(4) Similarly, using Lemma 6.3 (5).

(5) Using points (3) and (1) we have:

$$
\langle p ?\rangle(q) \otimes\langle p ?\rangle\left(q^{\perp}\right)=\langle p ?\rangle\left(q \otimes q^{\perp}\right)=\langle p ?\rangle(1)=p .
$$

Hence $\langle p ?\rangle(q) \leq p$. 
(6) Assume $q_{1} \leq q_{2}$, say via $q_{1} \otimes r=q_{2}$. Then, again using point (3) we get:

$$
\langle p ?\rangle\left(q_{1}\right) \otimes\langle p ?\rangle(r)=\langle p ?\rangle\left(q_{1} \otimes r\right)=\langle p ?\rangle\left(q_{2}\right) .
$$

Hence $\langle p ?\rangle\left(q_{1}\right) \leq\langle p ?\rangle\left(q_{2}\right)$.

(7) Using the De Morgan duality between FstAnd and FstThen we get:

$$
\begin{aligned}
{\left.[p ?](q)=\left(\operatorname{instr}_{p}\right)^{*}\left(\text { FstThen }_{q}\right)\right) } & =\left(\operatorname{instr}_{p}\right)^{*}\left(\text { FstAnd }\left(q^{\perp}\right)^{\perp}\right) \\
& =\left(\left(\operatorname{instr}_{p}\right)^{*}\left(\text { FstAnd }\left(q^{\perp}\right)\right)\right)^{\perp}=\langle p ?\rangle\left(q^{\perp}\right)^{\perp} .
\end{aligned}
$$

(8) We define the bound $b: X \rightarrow(1+1)+1$ by $b=\left[\kappa_{1}+\mathrm{id}, \kappa_{1} \circ \kappa_{2}\right] \circ\left(q+\right.$ !) $\circ$ instr $_{p}$. It proves $\langle p ?\rangle(q) \perp p^{\perp}$ and $\langle p ?\rangle(q) \otimes p^{\perp}=[p ?](q)$.

(9) We use Assumption 2 (2) in:

$$
\begin{aligned}
\left\langle\left[p_{1}, p_{2}\right] ?\right\rangle(q) & =[q, 0] \circ \operatorname{instr}_{\left[p_{1}, p_{2}\right]} \\
& =[q, 0] \circ\left[\kappa_{1}+\kappa_{1}, \kappa_{2}+\kappa_{2}\right] \circ\left(\text { instr }_{p_{1}}+\text { instr }_{p_{2}}\right) \\
& =\left[\left[q \circ \kappa_{1}, 0 \circ \kappa_{1}\right] \circ \text { instr }_{p_{1}},\left[q \circ \kappa_{2}, 0 \circ \kappa_{2}\right] \circ \text { instr }_{p_{2}}\right] \\
& =\left[\left[q \circ \kappa_{1}, 0\right] \circ \text { instr }_{p_{1}},\left[q \circ \kappa_{2}, 0\right] \circ \text { instr }_{p_{2}}\right] \\
& =\left[\left\langle p_{1} ?\right\rangle\left(q \circ \kappa_{1}\right),\left\langle p_{2} ?\right\rangle\left(q \circ \kappa_{2}\right)\right]
\end{aligned}
$$

(10) If $f: Y \rightarrow X$ is pure, then for $p, q \in \operatorname{Pred}(X)$,

$$
\begin{aligned}
f^{*}(\langle p ?\rangle(q))=[q, 0] \circ \operatorname{instr}_{p} \circ f & =[q, 0] \circ(f+f) \circ \text { instr }_{p \circ f} \quad \text { see Definition 8.9 } \\
& =[q \circ f, 0] \circ \operatorname{instr}_{f^{*}(p)} \\
& =\left\langle f^{*}(p) ?\right\rangle\left(f^{*}(q)\right) .
\end{aligned}
$$

There are some further properties of test operators that we do not list in Lemma 9.3 but describe separately because they deserve more attention. We first consider the substitution operation $f^{*}=\operatorname{Pred}(f)=w p(f)=(-) \circ f$ from Proposition 4.4 (2) that makes the mapping $X \mapsto \operatorname{Pred}(X)$ functorial. We show that for the special case where the map $f$ is an instrument instr $p$, substitution can be defined in logical terms. This result will be useful immediately, in a subsequent lemma, but may also be useful for Lindenbaum models where the logic has test operators.

The result will be formulated first for predicates (2-tests), and then more generally for $n$-tests. We separately mention version for $n=2$ because it has an easier proof and because it is useful on its own. It describes a form of "if-then-else".

Proposition 9.4. Substitution for instruments, in a category satisfying Assumption 2, can be described as follows.

(1) For predicates $p: X \rightarrow 1+1$ and $q: X+X \rightarrow 1+1$,

$$
\left(\operatorname{instr}_{p}\right)^{*}(q)=\langle p ?\rangle\left(q \circ \kappa_{1}\right) \otimes\left\langle p^{\perp} ?\right\rangle\left(q \circ \kappa_{2}\right)
$$

(2) For an $n$-test $p: X \rightarrow n \cdot 1$, where $n \geq 2$, and a predicate $q: n \cdot X \rightarrow 1+1$ one has:

$$
\left(\operatorname{instr}_{p}\right)^{*}(q)=\left\langle p_{1} ?\right\rangle\left(q_{1}\right) \otimes \cdots \otimes\left\langle p_{n} ?\right\rangle\left(q_{n}\right),
$$

for predicates $p_{i}$ corresponding to the $n$-test $p$ as in Lemma 4.11 - via $p_{i}=\triangleright_{i} \circ$ $p: X \rightarrow 1+1$, with $n$-ary partial projections $\triangleright_{i}$ like in (4.1) - and predicates $q_{i}=q \circ$ $\kappa_{i}: X \rightarrow 1+1$ on $X$. 
Proof. For the first point we calculate:

$$
\begin{array}{rlr}
\left(\text { instr }_{p}\right)^{*}(q) & =\left(\text { instr }_{p}\right)^{*}\left(\left[q \circ \kappa_{1}, q \circ \kappa_{2}\right]\right) & \\
= & \left(\text { instr }_{p}\right)^{*}\left(\left[q \circ \kappa_{1}, 0\right] \otimes\left[0, q \circ \kappa_{2}\right]\right) & \text { by (6.2) } \\
= & \left(\text { instr }_{p}\right)^{*}\left(\left[q \circ \kappa_{1}, 0\right]\right) \otimes\left(\text { instr }_{p}\right)^{*}\left(\left[0, q \circ \kappa_{2}\right]\right) & \\
& \quad \text { since substitution is a map of effect algebras } & \\
= & \langle p ?\rangle\left(q \circ \kappa_{1}\right) \otimes\left(\text { instr }_{p}\right)^{*}\left(\left[q \circ \kappa_{2}, 0\right] \circ\left[\kappa_{2}, \kappa_{1}\right]\right) & \\
= & \langle p ?\rangle\left(q \circ \kappa_{1}\right) \otimes\left(\left[\kappa_{2}, \kappa_{1}\right] \circ \text { instr }_{p}\right)^{*}\left(\left[q \circ \kappa_{2}, 0\right]\right) & \\
= & \langle p ?\rangle\left(q \circ \kappa_{1}\right) \otimes\left(\text { instr }_{p^{\perp}}\right)^{*}\left(\left[q \circ \kappa_{2}, 0\right]\right) & \text { by Assur } \\
= & \langle p ?\rangle\left(q \circ \kappa_{1}\right) \otimes\left\langle p^{\perp} ?\right\rangle\left(q \circ \kappa_{2}\right) .
\end{array}
$$

For the second point we need to use Assumption 2 (3) and also (1). The proof involves some bookkeeping with coprojections. We use the maps:

$$
\begin{aligned}
\triangleright_{i} & =\underbrace{\left[\kappa_{2}, \ldots, \kappa_{2}, \kappa_{1}, \kappa_{2}, \ldots, \kappa_{2}\right]}_{\kappa_{1} \text { at the } i \text {-the position }}: n \cdot 1 \longrightarrow 1+1 \\
\operatorname{swap}_{i} & =\underbrace{\left[\kappa_{i}, \kappa_{2}, \kappa_{3}, \ldots, \kappa_{i-1}, \kappa_{1}, \kappa_{i+1}, \ldots, \kappa_{n}\right]}_{\kappa_{i} \text { at the first, } \kappa_{1} \text { at the } i \text {-the position }}: n \cdot Y \cong n \cong Y .
\end{aligned}
$$

Then we can write $p_{i}=\triangleright_{i} \circ p=(\mathrm{id}+!) \circ \operatorname{swap}_{i} \circ p$, where id $+!: n \cdot 1=1+(n-1) \cdot 1 \rightarrow 1+1$. Hence we get:

$$
\begin{aligned}
\emptyset_{i}\left\langle p_{i} ?\right\rangle\left(q_{i}\right) & =\bigotimes_{i}\left[q \circ \kappa_{i}, \kappa_{2} \circ !\right] \circ \operatorname{instr}_{p_{i}} \\
& =\bigotimes_{i}\left[q \circ \kappa_{i}, \kappa_{2}\right] \circ(\mathrm{id}+!) \circ \operatorname{instr}_{(\mathrm{id}+!) \circ \operatorname{swap}_{i} \circ p} \\
& =\bigotimes_{i}\left[q \circ \kappa_{i}, \kappa_{2}\right] \circ(\mathrm{id}+!) \circ \operatorname{instr}_{\text {swap }_{i} \circ p} \\
& =\bigotimes_{i}\left[q \circ \kappa_{i}, \kappa_{2} \circ !\right] \circ \operatorname{swap}_{i} \circ \operatorname{instr}_{p} \\
& =\bigotimes_{i}\left[0, \ldots, 0, q \circ \kappa_{i}, 0, \ldots, 0\right] \circ \operatorname{instr}_{p} \\
& =\bigotimes_{i}\left(\operatorname{instr}_{p}\right)^{*}\left(\left[0, \ldots, 0, q \circ \kappa_{i}, 0, \ldots, 0\right]\right) \\
& =\left(\operatorname{instr}_{p}\right)^{*}\left(\emptyset_{i}\left[0, \ldots, 0, q \circ \kappa_{i}, 0, \ldots, 0\right]\right) \\
& =\left(\operatorname{instr}_{p}\right)^{*}\left(\left[q \circ \kappa_{1}, \ldots, q \circ \kappa_{n}\right]\right) \\
& =\left(\operatorname{instr}_{p}\right)^{*}(q) .
\end{aligned}
$$

by Assumption 2 (3) by Assumption 2 (1)

by 6.2

We obtain a consequence that highlights the role of side-effects in formulas. In an informal reading the result below says: the sum of ' $p$ andthen $q$ ', and of ' $p \perp$ andthen $q$ ' is the same as just having $q$. But in our present setting we have to take side-effect $\nabla \circ$ instr $_{p}$ of $p$ into account.

Corollary 9.5. For predicates $p, q: X \rightarrow 1+1$ in a category satisfying Assumption Q one has:

$$
\langle p ?\rangle(q) \otimes\left\langle p^{\perp} ?\right\rangle(q)=q \circ \nabla \circ \text { instr }_{p}
$$

We recall from Definition 8.1 that the map $\nabla \circ$ instr $_{p}$ is the side-effect of the predicate $p$. In case $p$ is side-effect-free, that is, in case $\nabla \circ$ instr $_{p}=\mathrm{id}$, the above equation (9.2) reduces to the standard result $\langle p ?\rangle(q) \otimes\left\langle p^{\perp} ?\right\rangle(q)=q$. 
Proof. Directly from Proposition 9.4 (11) we obtain:

$$
\begin{aligned}
\langle p ?\rangle(q) \otimes\left\langle p^{\perp} ?\right\rangle(q) & =\langle p ?\rangle\left([q, q] \circ \kappa_{1}\right) \otimes\left\langle p^{\perp} ?\right\rangle\left([q, q] \circ \kappa_{2}\right) \\
& =\left(\operatorname{instr}_{p}\right)^{*}([q, q]) \\
& =[q, q] \circ \text { instr }_{p} \\
& =q \circ \nabla \circ \text { instr }_{p} .
\end{aligned}
$$

We add another result illustrating that substitution $f^{*}(q)=q \circ f$ can be understood as weakest precondition $w p(f)(q)$. We apply this to the (binary case of the) test map from (7.6). It yields a formula that looks very much like the traditional weakest precondition formula for if-then-else:

$$
w p\left(\text { if } p \text { then } f_{1} \text { else } f_{2}\right)(q)=\left(p \wedge w p\left(f_{1}\right)(q)\right) \vee\left(\neg p \wedge w p\left(f_{2}\right)(q)\right) .
$$

In the present context the conjunctions are replaced by test operators that deal with the side-effects involved.

Lemma 9.6. For parallel maps $f_{1}, f_{2}: X \rightarrow Y$ in a category satisfying Assumption 2 and a predicate $p: X \rightarrow 1+1$ on $X$ we have:

$$
{ }_{w p}\left(p ?\left[f_{1}, f_{2}\right]\right)(q)=\langle p ?\rangle\left(w p\left(f_{1}\right)(q)\right) \otimes\left\langle p^{\perp} ?\right\rangle\left(w p\left(f_{2}\right)(q)\right) .
$$

Proof. We use Proposition 9.4 (11) to obtain:

$$
\begin{aligned}
\langle p ?\rangle\left(w p\left(f_{1}\right)(q)\right) \otimes\left\langle p^{\perp} ?\right\rangle\left(w p\left(f_{2}\right)(q)\right) & =\left(\operatorname{instr}_{p}\right)^{*}\left(\left[w p\left(f_{1}\right)(q), w p\left(f_{2}\right)(q)\right]\right) \\
& =\left[q \circ f_{1}, q \circ f_{2}\right] \circ \operatorname{instr}_{p} \\
& =q \circ\left[f_{1}, f_{2}\right] \circ \text { instr }_{p} \\
\stackrel{(7.6)}{=} & q \circ p ?\left[f_{1}, f_{2}\right] \\
& =w p\left(p ?\left[f_{1}, f_{2}\right]\right)(q) .
\end{aligned}
$$

We continue with $C^{*}$-algebras as instance. The definition of commutativity $\langle p ?\rangle(q)=\langle q$ ? $\rangle(p)$ used in Definition 9.1 turns out to coincide with commutativity in $C^{*}$-algebras. This is a nontrivial result that goes back to [32. It uses the Fuglede-Putnam-Rosenblum Theorem [73] for $C^{*}$-algebras. This theorem says: for normal elements $a, b$ one has for each $x$,

$$
a \cdot x=x \cdot b \Longrightarrow a^{*} \cdot x=x \cdot b^{*} .
$$

We recall that $a$ is normal if $a \cdot a^{*}=a^{*} \cdot a$.

Proposition 9.7. $A C^{*}$-algebra $A$ is commutative, in the sense that its multiplication. is commutative, if and only if it is commutative, in the sense of Definition 0.1: $\langle e ?\rangle(d)=$ $\langle d ?\rangle(e)$ holds for all effects $e, d \in[0,1]_{A}$.

Proof. First assume that multiplication - of the $C^{*}$-algebra $A$ is commutative. Then:

$$
\langle e ?\rangle(d)=\sqrt{e} \cdot d \cdot \sqrt{e}=\sqrt{e} \cdot \sqrt{e} \cdot d=e \cdot d=e \cdot \sqrt{d} \cdot \sqrt{d}=\sqrt{d} \cdot e \cdot \sqrt{d}=\langle d ?\rangle(e) .
$$

In the other direction, it suffices by Lemma 8.6 to prove $e \cdot d=d \cdot e$ for effects $e, d$. By assumption we have $\sqrt{e} \cdot d \cdot \sqrt{e}=\langle e ?\rangle(d)=\langle d ?\rangle(e)=\sqrt{d} \cdot e \cdot \sqrt{d}$. The product $\sqrt{e} \cdot \sqrt{d}$ is 
normal since:

$$
\begin{aligned}
(\sqrt{e} \cdot \sqrt{d})^{*} \cdot(\sqrt{e} \cdot \sqrt{d}) & =\sqrt{d} \cdot \sqrt{e} \cdot \sqrt{e} \cdot \sqrt{d} \\
& =\sqrt{d} \cdot e \cdot \sqrt{d} \\
& =\sqrt{e} \cdot d \cdot \sqrt{e} \quad \text { by assumption } \\
& =\sqrt{e} \cdot \sqrt{d} \cdot \sqrt{d} \cdot \sqrt{e} \\
& =(\sqrt{e} \cdot \sqrt{d}) \cdot(\sqrt{e} \cdot \sqrt{d})^{*} .
\end{aligned}
$$

We use Fuglede-Putnam-Rosenblum (9.3) with $a=\sqrt{e} \cdot \sqrt{d}, b=\sqrt{d} \cdot \sqrt{e}$ and $x=\sqrt{e}$. The antecedent holds, since $a \cdot x=\sqrt{e} \cdot \sqrt{d} \cdot \sqrt{e}=x \cdot b$. It yields as conclusion:

$$
\sqrt{d} \cdot e=(\sqrt{e} \cdot \sqrt{d})^{*} \cdot \sqrt{e} \stackrel{\sqrt{9.3}}{=} \sqrt{e} \cdot(\sqrt{d} \cdot \sqrt{e})^{*}=e \cdot \sqrt{d} .
$$

But then we are done since:

$$
e \cdot d=e \cdot \sqrt{d} \cdot \sqrt{d}=\sqrt{d} \cdot e \cdot \sqrt{d}=\sqrt{d} \cdot \sqrt{d} \cdot e=d \cdot e .
$$

We conclude this section with an example that illustrates the use of the logical test operators in an elementary probability calculation.

Example 9.8. The idea in the famous polarisation experiment is to send photons polarised as $|\uparrow\rangle=|0\rangle=\left(\begin{array}{l}1 \\ 0\end{array}\right)$ through filters.

(1) If these photons hit one filter with polarisation $|\rightarrow\rangle=|1\rangle=\left(\begin{array}{l}0 \\ 1\end{array}\right)$, then nothing passes.

(2) Next assume there are two consecutive filters: first the $|\uparrow\rangle$-photons have to go through a filter with polarisation $|\nearrow\rangle=\frac{1}{\sqrt{2}}\left(\begin{array}{l}1 \\ 1\end{array}\right)$, and then through a filter with polarisation $|\rightarrow\rangle=|1\rangle=\left(\begin{array}{l}0 \\ 1\end{array}\right)$. Surprisingly, now one quarter of the original photons get through. We refer to e.g. [72] for an explanation, and focus here on a calculation of this probability $\frac{1}{4}$ in the current quantitative logic.

For these experiments we use the $C^{*}$-algebra $A=\mathcal{B}\left(\mathbb{C}^{2}\right)$ with initial state $\omega_{\uparrow}: A \rightarrow \mathbb{C}$ given by $\omega_{\uparrow}(M)=\langle 0|M| 0\rangle$. The effect $p_{\rightarrow} \in[0,1]_{A}=\mathcal{E} f\left(\mathbb{C}^{2}\right)$ corresponding to the filter with polarisation $|\rightarrow\rangle$ is given by:

$$
p_{\rightarrow}=|\rightarrow\rangle\langle\rightarrow|=\left(\begin{array}{ll}
0 & 1
\end{array}\right)\left(\begin{array}{l}
0 \\
1
\end{array}\right)=\left(\begin{array}{ll}
0 & 0 \\
0 & 1
\end{array}\right) .
$$

It is clearly a projection. Evaluating this predicate in the initial state $\omega_{\uparrow}$ yields the probability:

$$
\omega_{\uparrow} \models p_{\rightarrow} \stackrel{5.11}{=} \omega_{\uparrow}\left(p_{\rightarrow}\right)=\left\langle\uparrow\left|p_{\rightarrow}\right| \uparrow\right\rangle=\left(\begin{array}{ll}
1 & 0
\end{array}\right)\left(\begin{array}{ll}
0 & 0 \\
0 & 1
\end{array}\right)\left(\begin{array}{l}
1 \\
0
\end{array}\right)=0 .
$$

This is the probability of the above first experiment: nothing goes through.

Things become more interesting in the second experiment, where we first send the $|0\rangle$-photon through the filter with polarisation $|\nearrow\rangle$. This involves the effect:

$$
p_{\nearrow}=|\nearrow\rangle\langle\nearrow|=\frac{1}{\sqrt{2}}\left(\begin{array}{l}
1 \\
1
\end{array}\right) \frac{1}{\sqrt{2}}\left(\begin{array}{ll}
1 & 1
\end{array}\right)=\frac{1}{2}\left(\begin{array}{ll}
1 & 1 \\
1 & 1
\end{array}\right) .
$$

This $p_{\nearrow}$ is also a projection, so that $\sqrt{p_{\nearrow}}=p_{\nearrow}$.

We now compute the probability via the test operators from Section 9 First going through the filter $\nearrow$ and then through $\rightarrow$ is expressed via the test-andthen operator. The resulting probability is:

$$
\begin{aligned}
\omega_{\uparrow} \models\left\langle p_{\nearrow} ?\right\rangle\left(p_{\rightarrow}\right) \stackrel{\text { 9.1 }}{=}\left\langle\uparrow\left|\sqrt{p_{\nearrow}} p_{\rightarrow} \sqrt{p_{\nearrow}}\right| \uparrow\right\rangle \\
=\left(\begin{array}{lll}
1 & 0
\end{array}\right) \frac{1}{2}\left(\begin{array}{ll}
1 & 1 \\
1 & 1
\end{array}\right)\left(\begin{array}{ll}
0 & 0 \\
0 & 1
\end{array}\right) \frac{1}{2}\left(\begin{array}{ll}
1 & 1 \\
1 & 1
\end{array}\right)\left(\begin{array}{l}
1 \\
0
\end{array}\right)=\frac{1}{4}\left(\begin{array}{ll}
1 & 0
\end{array}\right)\left(\begin{array}{ll}
1 & 1 \\
1 & 1
\end{array}\right)\left(\begin{array}{l}
1 \\
0
\end{array}\right)=\frac{1}{4} .
\end{aligned}
$$


Instead of the andthen-test we can also use the weaker then-test. It adds the option that the photon does not get through the first filter - which happens with probability $\frac{1}{2}$. Then:

$$
\begin{aligned}
& \omega_{\uparrow} \models\left[p_{\nearrow} ?\right]\left(p_{\rightarrow}\right) \stackrel{\text { (9.1) }}{=}\left\langle\uparrow\left|\left(\sqrt{p_{\nearrow}} p_{\rightarrow} \sqrt{p_{\nearrow}}+1-p_{\nearrow}\right)\right| \uparrow\right\rangle \\
& =\left(\begin{array}{ll}
1 & 0
\end{array}\right)\left(\frac{1}{2}\left(\begin{array}{ll}
1 & 1 \\
1 & 1
\end{array}\right)\left(\begin{array}{ll}
0 & 0 \\
0 & 1
\end{array}\right) \frac{1}{2}\left(\begin{array}{ll}
1 & 1 \\
1 & 1
\end{array}\right)+\left(\begin{array}{ll}
1 & 0 \\
0 & 1
\end{array}\right)-\frac{1}{2}\left(\begin{array}{ll}
1 & 1 \\
1 & 1
\end{array}\right)\right)\left(\begin{array}{l}
1 \\
0
\end{array}\right) \\
& =\left(\begin{array}{ll}
1 & 0
\end{array}\right)\left(\frac{1}{4}\left(\begin{array}{ll}
1 & 1 \\
1 & 1
\end{array}\right)+\frac{1}{2}\left(\begin{array}{cc}
1 & -1 \\
-1 & 1
\end{array}\right)\right)\left(\begin{array}{l}
1 \\
0
\end{array}\right) \\
& =\left(\begin{array}{ll}
1 & 0
\end{array}\right) \frac{1}{4}\left(\begin{array}{cc}
3 & -1 \\
-1 & 3
\end{array}\right)\left(\begin{array}{l}
1 \\
0
\end{array}\right) \\
& =\frac{3}{4} \text {. }
\end{aligned}
$$

\section{AdDing TENSOR PRODUCTS}

Our third assumption involves tensor products, with some special properties. We recall that a symmetric monoidal structure on a category $\mathbf{B}$ involves a functor $\otimes: \mathbf{B} \times \mathbf{B} \rightarrow \mathbf{B}$ and tensor unit $I \in \mathbf{B}$, together with canonical isomorphisms:

$$
X \otimes(Y \otimes Z) \stackrel{\alpha}{\cong}(X \otimes Y) \otimes Z \quad X \otimes I \stackrel{\rho}{\cong} X \quad X \otimes Y \stackrel{\gamma}{\cong} Y \otimes X
$$

One often writes $\lambda=\rho \circ \gamma: I \otimes X \stackrel{\cong}{\longrightarrow}$. These isomorphisms make some obvious diagrams commute, see [60].

Assumption 3. Our category satisfies not only Assumptions 1 and 2 but is also symmetric monoidal with the additional requirements described in the next five points.

(1) The final object 1 is the tensor unit. As a result we have a 'tensor with projections', since projections $\pi_{1}, \pi_{2}$ can be defined in:

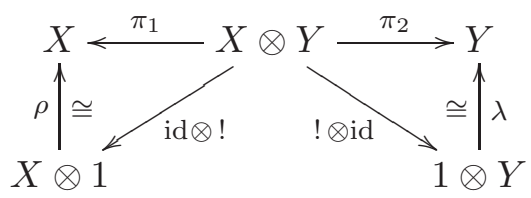

These projections $\pi_{1}, \pi_{2}$ are natural in $X$ and $Y$.

(2) The tensor product $\otimes$ distributes over finite coproducts. Explicitly, the following canonical maps are isomorphisms:

$$
(X \otimes A)+(Y \otimes A) \underset{\left[\kappa_{1} \otimes \mathrm{id}, \kappa_{2} \otimes \mathrm{id}\right]}{\cong}(X+Y) \otimes A \quad 0 \underset{!}{\stackrel{!}{\longrightarrow}} A \otimes 0
$$

We shall write $\theta_{1}=\left[\kappa_{1} \otimes \mathrm{id}, \kappa_{2} \otimes \mathrm{id}\right]^{-1}$ for the inverse of this map on the left. There is a similar distributivity map $\theta_{2}: A \otimes(X+Y) \stackrel{\cong}{\longrightarrow}(A \otimes X)+(A \otimes Y)$ in the other coordinate. These distributivity maps extend to $n$-ary coproducts.

(3) The monoidal isomorphisms (10.1) are pure — see Definition 8.9.

(4) If both $f, g$ are pure maps, then $f \otimes g$ is pure too.

(5) For each $n$-test $p: X \rightarrow n \cdot 1$ and for each object $A$ the following diagrams commute.
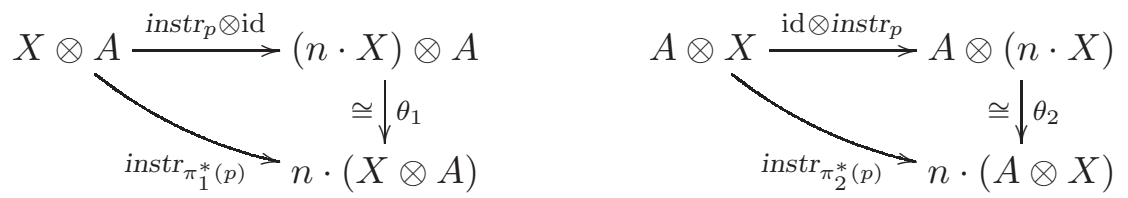
This concludes our assumptions about tensors.

The projections $\pi_{1}, \pi_{2}$ in (10.2) for the tensor $\otimes$, like in [36], enable discarding of resources. There are in general no associated diagonals $X \rightarrow X \otimes X$ because of the nocloning Theorem [83, 22, 1].

The two distributivity isomorphisms $\theta_{1}, \theta_{2}$ interact in the following way.

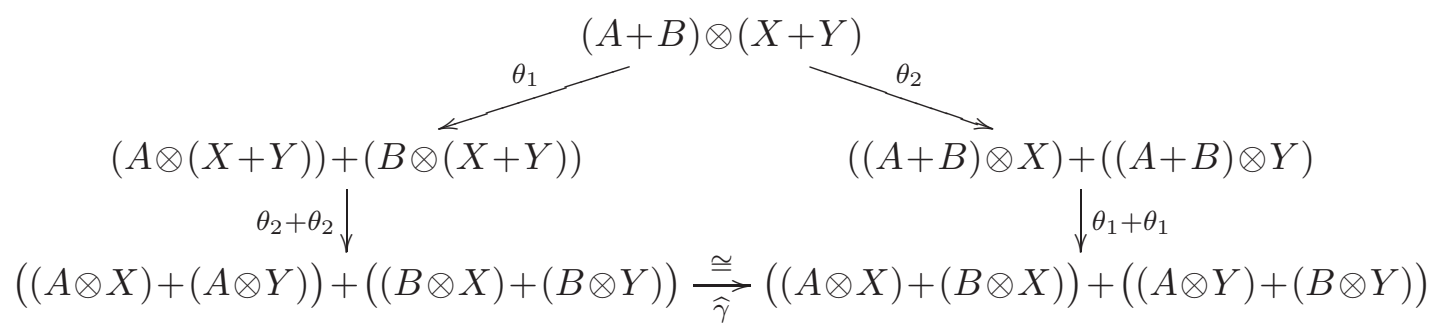

where $\widehat{\gamma}=\left[\kappa_{1}+\kappa_{1}, \kappa_{2}+\kappa_{2}\right]$ is the map that swaps the inner two occurrences; it is its own inverse.

Sometimes one writes $X^{\otimes n}=X \otimes \cdots \otimes X$ for the $n$-fold tensor. Because $\otimes$ distributes over + and 1 is the tensor unit, we have for the object $2=1+1$ the following isomorphism:

$$
2^{\otimes n}=\underbrace{2 \otimes \cdots \otimes 2}_{n \text { times }} \cong \underbrace{1+\cdots+1}_{2^{n} \text { times }}=2^{n} .
$$

Further we have $n \cdot X \cong X \otimes(n \cdot 1)$, since:

$$
n \cdot X \cong n \cdot(X \otimes 1) \cong X \otimes(n \cdot 1)
$$

Hence, in presence of tensors we may describe instruments also as maps $X \rightarrow X \otimes(n \cdot 1)$. The side-effect is then obtained via the composite $X \rightarrow X \otimes(n \cdot 1) \rightarrow X$, using the the first projection, as in point (10.2).

Examples 10.1. In the category Sets, or in any extensive category, we can use cartesian products $\times$ as tensors, see Appendix A.1. They obviously distribute over coproducts. In the Kleisli category $\mathcal{K} \ell(\mathcal{D})$ the projections $\pi_{i}: X_{1} \times X_{2} \rightarrow \mathcal{D}\left(X_{i}\right)$ are given by $\pi_{i}\left(x_{1}, x_{2}\right)=1\left|x_{i}\right\rangle$. It is not hard to check that instruments in $\mathcal{K} \ell(\mathcal{D})$ commute with distributivity maps, as in diagrams (10.4).

Tensors of $C^{*}$-algebras are a delicate matter. They exist in the category of $C^{*}$-algebras with completely positive maps. Hence they also exist on $\left(\mathbf{C s t a r}_{\mathrm{CPU}}\right)^{\mathrm{op}}$, and the "minimal" tensor even distributes over finite coproducts, as shown in [15, see Appendix A.4.

We need to check that our previous Assumptions 1 and 2 also hold in $\mathbf{C s t a r}_{\mathrm{CPU}}$. This is not a problem. A useful fact is: when either $A$ or $B$ is a commutative $C^{*}$ algebra, then a positive unital map $f: A \rightarrow B$ is automatically completely positive. Hence our predicates and tests $\mathbb{C}^{n} \rightarrow A$ are completely positive. The instrument instr $\vec{e}_{\vec{e}}$ from (7.5) is also completely positive, due to its special form.

The projection $A \otimes B \rightarrow A$ in $\left(\mathbf{C s t a r}_{\mathrm{CPU}}\right)^{\mathrm{op}}$, described as a map $A \rightarrow A \otimes B$ in Cstar $_{\mathrm{CPU}}$, is given by $a \mapsto a \otimes 1$. When working in Cstar $_{\mathrm{CPU}}$ we often call this map a coprojection, for obvious reasons, and write it as $\kappa_{1}$.

We check the interaction between instruments and distributivity from diagrams (10.4) in the binary case (for $n=2$ ). So assume we have a test $e \in[0,1]_{A}$, with associated instrument $\operatorname{instr}_{e}: A \times A \rightarrow A$ given by $\operatorname{instr}_{e}\left(a_{1}, a_{2}\right)=\sqrt{e} \cdot a_{1} \cdot \sqrt{e}+\sqrt{1-e} \cdot a_{2} \cdot \sqrt{1-e}$. 
We get a predicate $\kappa_{1}^{*}(e)=e \otimes 1 \in[0,1]_{A \otimes B}$. Since $(\sqrt{e} \otimes 1) \cdot(\sqrt{e} \otimes 1)=(e \otimes 1)$ we have $\sqrt{e \otimes 1}=(\sqrt{e} \otimes 1)$. Similarly for $1-e$ we have:

$$
(\sqrt{1-e} \otimes 1) \cdot(\sqrt{1-e} \otimes 1)=(1-e) \otimes 1=1 \otimes 1-e \otimes 1=1-e \otimes 1,
$$

so that $\sqrt{1-(e \otimes 1)}=\sqrt{1-e} \otimes 1$. Now we can prove (10.4):

$$
\begin{aligned}
& \left(\operatorname{instr}_{\kappa_{1}^{*}(e)} \circ \theta_{1}\right)\left(\left(a_{1}, a_{2}\right) \otimes b\right) \\
& =\operatorname{instr}_{\kappa_{1}^{*}(e)}\left(a_{1} \otimes b, a_{2} \otimes b\right) \\
& =\sqrt{e \otimes 1} \cdot\left(a_{1} \otimes b\right) \cdot \sqrt{e \otimes 1}+\sqrt{1-(e \otimes 1)} \cdot\left(a_{2} \otimes b\right) \cdot \sqrt{1-(e \otimes 1)} \\
& =(\sqrt{e} \otimes 1) \cdot\left(a_{1} \otimes b\right) \cdot(\sqrt{e} \otimes 1)+(\sqrt{1-e} \otimes 1) \cdot\left(a_{2} \otimes b\right) \cdot(\sqrt{1-e} \otimes 1) \\
& =\left(\sqrt{e} \cdot a_{1} \cdot \sqrt{e}\right) \otimes b+\left(\sqrt{1-e} \cdot a_{2} \cdot \sqrt{1-e}\right) \otimes b \\
& =\left(\sqrt{e} \cdot a_{1} \cdot \sqrt{e}+\sqrt{1-e} \cdot a_{2} \cdot \sqrt{1-e}\right) \otimes b \\
& =\operatorname{instr}_{e}\left(a_{1}, a_{2}\right) \otimes b \\
& =\left(\operatorname{instr}_{e} \otimes \text { id }\right)\left(\left(a_{1}, a_{2}\right) \otimes b\right) .
\end{aligned}
$$

The object $2=1+1$ in a category satisfying Assumption 3 carries a special multiplication map $m: 2 \otimes 2 \rightarrow 2$, namely $m=$ [id, $\left.\kappa_{2} \circ !\right] \circ(\rho+\rho) \circ \theta_{2}$, obtained by going east-south-west in:

$$
\begin{aligned}
(1+1) & \otimes(1+1) \underset{\theta_{2}=\left[\mathrm{id} \otimes \kappa_{1}, \operatorname{id} \otimes \kappa_{2}\right]^{-1}}{\cong}((1+1) \otimes 1) \\
& m \downarrow \\
& \begin{array}{c}
\cong \\
1
\end{array}
\end{aligned}
$$

Proposition 10.2. Let $\boldsymbol{B}$ be a category satisfying Assumption 3 .

(1) The map $m: 2 \otimes 2 \rightarrow 2$ defined in (10.6) makes $2=1+1 \in \boldsymbol{B}$ into a commutative monoid in $\boldsymbol{B}$, with unit scalar $1=\kappa_{1}: 1 \rightarrow 1+1=2$ as multiplicative unit, and zero scalar $0=\kappa_{2}: 1 \rightarrow 2$ as zero element.

(2) Multiplication of scalars from Lemma 3.3 can be expressed via this multiplication map $m$, namely as:

$$
s \cdot r=\left(1 \stackrel{\lambda^{-1}=\rho^{-1}}{\cong} 1 \otimes 1 \stackrel{s \otimes r}{\longrightarrow} 2 \otimes 2 \stackrel{m}{\longrightarrow} 2\right) .
$$

(3) This multiplication - of scalars is then also commutative: $s \cdot r=r \cdot s$. Thus, in presence of tensors, the scalars Pred(1) form a commutative monoid.

Recall from Section $\left[5\right.$ the distribution monad $\mathcal{D}_{M}$ : Sets $\rightarrow$ Sets associated with the effect monoid $M=\operatorname{Pred}(1)$. This monad is commutative if $M=\operatorname{Pred}(1)$ is commutative, see [39]. By a general categorical result of Kock [54, 53] — "commutative theories have tensors" - this implies that the category $\operatorname{Conv}_{M}=\mathcal{E} \mathcal{M}\left(\mathcal{D}_{M}\right)$ of Eilenberg-Moore algebras is symmetric monoidal closed. 
Proof. For the first point we concentrate on commutativity, which is the most interesting part. We need to prove $m \circ \gamma=m$. First consider the diagram:

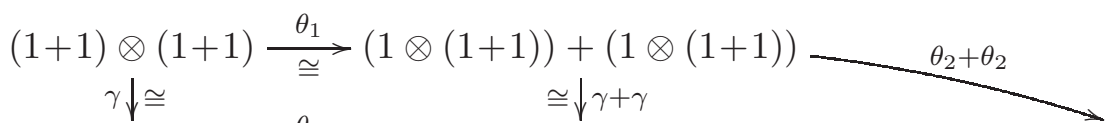

$$
\begin{aligned}
& (1+1) \otimes(1+1) \underset{\theta_{2}}{\stackrel{\longrightarrow}{\cong}}((1+1) \otimes 1)+((1+1) \otimes 1) \quad((1 \otimes 1)+(1 \otimes 1))+((1 \otimes 1)+(1 \otimes 1)) \\
& m \downarrow \quad \cong \downarrow \rho+\rho
\end{aligned}
$$

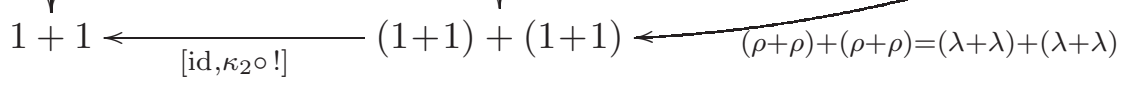

Now we can use the interaction between $\theta_{2}$ and $\theta_{1}$ from (10.5) in:

$$
\begin{aligned}
m \circ \gamma & =\left[\mathrm{id}, \kappa_{2} \circ !\right] \circ(\rho+\rho) \circ \theta_{2} \circ \gamma \\
& =\left[\mathrm{id}, \kappa_{2} \circ !\right] \circ((\rho+\rho)+(\rho+\rho)) \circ\left(\theta_{2}+\theta_{2}\right) \circ \theta_{1} \\
\stackrel{10.5}{=} & {\left[\mathrm{id}, \kappa_{2} \circ !\right] \circ((\rho+\rho)+(\rho+\rho)) \circ \widehat{\gamma} \circ\left(\theta_{1}+\theta_{1}\right) \circ \theta_{2} } \\
& =\left[\mathrm{id}, \kappa_{2} \circ !\right] \circ \widehat{\gamma} \circ((\rho+\rho)+(\rho+\rho)) \circ\left(\theta_{1}+\theta_{1}\right) \circ \theta_{2} \\
& =\left[\mathrm{id}, \kappa_{2} \circ !\right] \circ\left[\kappa_{1}+\kappa_{1}, \kappa_{2}+\kappa_{2}\right] \circ(\rho+\rho) \circ \theta_{2} \\
& =\left[\mathrm{id}, \kappa_{2} \circ !\right] \circ(\rho+\rho) \circ \theta_{2} \\
& =m .
\end{aligned}
$$

A crucial part of this proof is that $\lambda=\rho: 1 \otimes 1 \rightarrow 1$. This is like in the Eckmann-Hilton style argument in [52]. We also check that $\kappa_{1}: 1 \rightarrow 1+1=2$ is the unit for $m$ : Thus:

$$
\begin{aligned}
m \circ\left(\mathrm{id} \otimes \kappa_{1}\right) & =\left[\mathrm{id}, \kappa_{2} \circ !\right] \circ(\rho+\rho) \circ \theta_{2} \circ\left(\mathrm{id} \otimes \kappa_{1}\right) \\
& =\left[\mathrm{id}, \kappa_{2} \circ !\right] \circ(\rho+\rho) \circ \kappa_{1} \quad \text { since } \theta_{2}^{-1} \circ \kappa_{1}=\mathrm{id} \otimes \kappa_{1} \\
& =\left[\mathrm{id}, \kappa_{2} \circ !\right] \circ \kappa_{1} \circ \rho \\
& =\rho .
\end{aligned}
$$

Remaining steps to show that $m: 2 \otimes 2 \rightarrow 2$ is a monoid are left to the reader.

For the second point we first prove $s \cdot r=m \circ(s \otimes r) \circ \lambda^{-1}$ via the following diagram.

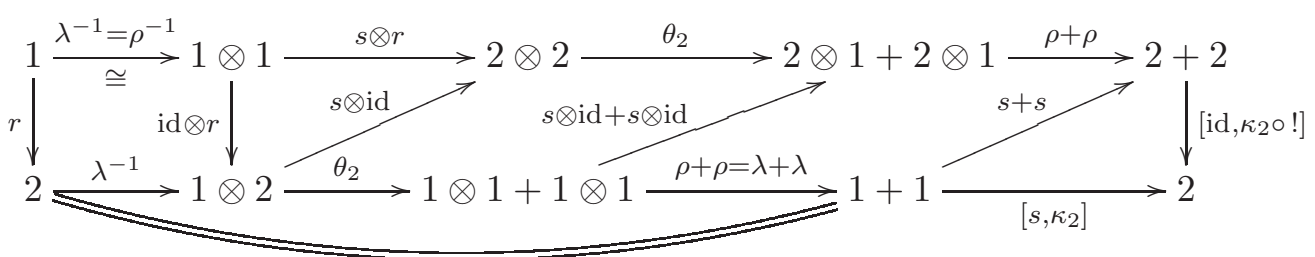

But now commutativity of the multiplication - of predicates follows from commutativity of $m$ in:

$$
\begin{aligned}
s \cdot r=m \circ(s \otimes r) \circ \lambda^{-1} & =m \circ \gamma \circ(s \otimes r) \circ \lambda^{-1} \quad \text { by the first point } \\
& =m \circ(r \otimes s) \circ \gamma \circ \lambda^{-1}=m \circ(r \otimes s) \circ \rho^{-1}=r \cdot s .
\end{aligned}
$$

Examples 10.3. We briefly review what the multiplication map $m: 2 \otimes 2 \rightarrow 2$ from (10.6) is in our main examples. In the category Sets we have $2=\{0,1\}$ with $m: 2 \times 2 \rightarrow 2$ given by multiplication, that is, by conjunction. Similarly, in the Kleisli category $\mathcal{K} \ell(\mathcal{D})$ of the distribution monad the map $m: 2 \times 2 \rightarrow \mathcal{D}(2) \cong[0,1]$ is multiplication. In the category of 
$C^{*}$-algebras this multiplication is a positive unital map $m: \mathbb{C}^{2} \rightarrow \mathbb{C}^{2} \otimes \mathbb{C}^{2}$, given on the two standard basis vectors $|0\rangle=\left(\begin{array}{l}1 \\ 0\end{array}\right)$ and $|1\rangle=\left(\begin{array}{l}0 \\ 1\end{array}\right)$ in $\mathbb{C}^{2}$ as:

$$
m(|0\rangle)=|0\rangle \otimes|0\rangle \quad \text { and } \quad m(|1\rangle)=|0\rangle \otimes|1\rangle+|1\rangle \otimes|0\rangle+|1\rangle \otimes|1\rangle .
$$

In the remainder of this section we consider projections and pairings, both for states and for predicates. We shall see that projections after pairings on states return the original results, but not the other way around. The latter precisely captures dependence and entanglement, see Example 10.9. We start with the projections described in (10.2).

Lemma 10.4. The projections $X \leftarrow X \otimes Y \rightarrow Y$ in (10.2) that can be defined in a category satisfying Assumption 3 are pure. As a consequence, also the unique maps $X \rightarrow 1$ to the terminal object are pure.

Proof. We show that the first projection $\pi_{1}: X \otimes Y \rightarrow X$ is pure. So let $p: X \rightarrow n \cdot 1$ be an $n$-test. We have to prove $\left(n \cdot \pi_{1}\right) \circ \operatorname{instr}_{\pi_{1}^{*}(p)}=\operatorname{instr}_{p} \circ \pi_{1}$, see Definition 8.9, We use requirement (5) in Assumption 3 as the upper part of the diagram:

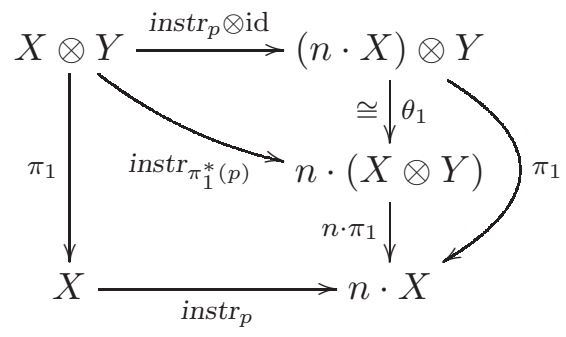

In this diagram we have $\left(n \cdot \pi_{1}\right) \circ \theta_{1}=\pi_{1}$ since:

$$
\pi_{1} \circ \theta_{1}^{-1}=\pi_{1} \circ\left[\kappa_{1} \otimes \mathrm{id}\right]_{i \leq n}=\left[\pi_{1} \circ\left(\kappa_{1} \otimes \mathrm{id}\right)\right]_{i \leq n}=\left[\kappa_{i} \circ \pi_{1}\right]_{i \leq n}=n \cdot \pi_{1} .
$$

But then we are done since by naturality of $\pi_{1}$ the outer diagram commutes. Finally, the unique map $X \rightarrow 1$ is pure, since it can be written as composite of pure maps:

$$
X \stackrel{\lambda^{-1}}{\cong} 1 \otimes X \stackrel{\pi_{1}}{\longrightarrow} 1
$$

In the sequel we restrict ourselves to the first projection $\pi_{1}: X \otimes Y \rightarrow X$, since the second one can be handled symmetrically. Via the predicate and state functors Pred and Stat from the state-and-effect triangle (5.4) we obtain maps (of effect modules and of convex sets):

$$
\operatorname{Pred}(X) \stackrel{\operatorname{Pred}\left(\pi_{1}\right)=\left(\pi_{1}\right)^{*}}{\longrightarrow} \operatorname{Pred}(X \otimes Y) \quad \operatorname{Stat}(X \otimes Y) \stackrel{\operatorname{Stat}\left(\pi_{1}\right)=\left(\pi_{1}\right)_{*}}{\longrightarrow} \operatorname{Stat}(X)
$$

The first operation $\left(\pi_{1}\right)^{*}$ sends a predicate on $X$ to a predicate on the extended type $X \otimes Y$. It corresponds to weakening in logic, that is, to addition of an unused variable. The second operation $\left(\pi_{1}\right)_{*}$ corresponds to taking the marginal, which, in the case of Hilbert spaces is the partial trace, see Lemma 10.5 below.

In the category Sets the first projection applied to a state $\omega=(x, y) \in X \times Y$ is simply the ordinary projection $\pi_{1} \omega=x \in X$. The situation is slightly more interesting in the Kleisli category $\mathcal{K} \ell(\mathcal{D})$ of the distribution monad. There, for a distribution $\omega \in \mathcal{D}(X \times Y)$, the projection $\pi_{1} \omega \in \mathcal{D}(X)$ is the marginal distribution, namely:

$$
\pi_{1} \omega(x)=\mathcal{D}\left(\pi_{1}\right)(\omega)(x)=\sum_{y \in Y} \omega(x, y) .
$$


In $\left(\mathbf{C s t a r}_{\mathrm{CPU}}\right)^{\text {op }}$ the left marginal of a state $\omega: A \otimes B \rightarrow \mathbb{C}$ is the map $\pi_{1} \omega: A \rightarrow \mathbb{C}$ given by $\pi_{1} \omega(a)=\omega(a \otimes 1)$. When the $C^{*}$-algebra is of the form $A=\mathcal{B}(\mathcal{H})$ for a finite-dimensional Hilbert space $\mathcal{H}$, we obtain the so-called partial trace.

Lemma 10.5. Let $\mathcal{H}, \mathcal{K}$ be two finite-dimensional Hilbert space. The left marginal, described on density matrices via the chain

$$
\mathcal{D M}(\mathcal{H} \otimes \mathcal{K}) \stackrel{\sqrt{5.3}}{\cong} \operatorname{Stat}(\mathcal{B}(\mathcal{H} \otimes \mathcal{K})) \stackrel{\text { A.4) }}{\cong} \operatorname{Stat}(\mathcal{B}(\mathcal{H}) \otimes \mathcal{B}(\mathcal{K})) \stackrel{\left(\pi_{1}\right)_{*}}{\longrightarrow} \operatorname{Stat}(\mathcal{B}(\mathcal{H})) \stackrel{\sqrt[5.3]{\cong}}{\cong} \mathcal{M}(\mathcal{H})
$$

is the partial trace operation $\rho \mapsto \operatorname{tr}_{\mathcal{K}}(\rho)$.

Proof. Each density matrix $\rho \in \mathcal{D M}(\mathcal{H} \otimes \mathcal{K})$ gives rise to a state $\omega_{\rho}: \mathcal{B}(\mathcal{H}) \otimes \mathcal{B}(\mathcal{K}) \rightarrow \mathbb{C}$ given by $\omega_{\rho}(M \otimes N)=\operatorname{tr}(\rho(M \otimes N))$. The resulting marginal $\pi_{1} \omega_{\rho}: \mathcal{B}(\mathcal{H}) \rightarrow \mathbb{C}$ is $\pi_{1} \omega_{\rho}(M)=$ $\operatorname{tr}(\rho(M \otimes \mathrm{id}))$. The partial $\operatorname{trace}_{\operatorname{tr}}(\rho) \in \mathcal{D} \mathcal{M}(\mathcal{H})$ precisely satisfies $\operatorname{tr}\left(\operatorname{tr}_{\mathcal{K}}(\rho) M\right)=\operatorname{tr}(\rho(M \otimes$ id)), see also [35, Defn. 2.68].

We continue with pairings.

Definition 10.6. Assume we are in category B satisfying Assumption 3. For two states $\omega_{1}: 1 \rightarrow X_{1}$ and $\omega_{2}: 1 \rightarrow X_{2}$ we write $\omega_{1} \otimes \omega_{2}: 1 \rightarrow X_{1} \otimes X_{2}$ for the state obtained by:

$$
\omega_{1} \odot \omega_{2}=\left(1 \stackrel{\lambda^{-1}=\rho^{-1}}{\longrightarrow} 1 \otimes 1 \stackrel{\omega_{1} \otimes \omega_{2}}{\longrightarrow} X_{1} \otimes X_{2}\right) .
$$

Similarly, for two predicates $p_{1}: X_{1} \rightarrow 1+1$ and $p_{2}: X_{2} \rightarrow 1+1$ we define a pairing predicate $p_{1} \odot p_{2}: X_{1} \otimes X_{2} \rightarrow 1+1$ as:

$$
p_{1} \odot p_{2}=\left(X_{1} \otimes X_{2} \stackrel{p_{1} \otimes p_{2}}{\longrightarrow} 2 \otimes 2 \stackrel{m}{\longrightarrow} 2\right),
$$

where $m: 2 \otimes 2 \rightarrow 2$ is the multiplication map from (10.6).

One can freely extend these pairing definitions to multiple states and predicates, like in $\omega_{1} \odot \cdots \odot \omega_{n}$ and $p_{1} \odot \cdots \odot p_{n}$, where we implicitly use associativity.

The next result presents some basic results about these pairings. The first one, for instance, could be useful for modular reasoning, where separate properties for separate states can be proved separately.

Lemma 10.7. The pairings on states and predicates defined above satisfy:

(1) $\left(\omega_{1} \odot \omega_{2} \models p_{1} \odot p_{2}\right)=m\left(\omega_{1} \models p_{1}, \omega_{2} \models p_{2}\right)$;

(2) $\pi_{i} \circ\left(\omega_{1} \odot \omega_{2}\right)=\omega_{i}$;

(3) the pairing $\odot$ on states is bi-affine, i.e. affine in each argument separately;

(4) weakening is pairing with truth: $\left(\pi_{1}\right)^{*}(p)=p \odot 1$, where $1=\kappa_{1} \circ$ ! is the truth predicate;

(5) the pairing $\odot$ on predicates is a bihomomorphism of effect modules: the mappings $p \odot(-)$ and $(-) \odot p$ preserve finite sums $\otimes, 0$ and scalar multiplication, and satisfy $1 \odot 1=1$.

Proof. The first point immediately holds, and for the second one we have:

$$
\pi_{1} \circ\left(\omega_{1} \odot \omega_{2}\right)=\rho \circ(\mathrm{id} \otimes !) \circ\left(\omega_{1} \otimes \omega_{2}\right) \circ \rho^{-1}=\rho \circ\left(\omega_{1} \otimes \mathrm{id}\right) \circ \rho^{-1}=\omega_{1} .
$$


We show that $\odot$ is bi-affine. Suppose we have an $n$-test $p: 1 \rightarrow n \cdot 1$, and $n$ states $\omega_{i}: 1 \rightarrow X$. Then for each state $\omega: 1 \rightarrow Y$,

$$
\begin{array}{rlrl}
\left(\emptyset_{i} p_{i} \omega_{i}\right) \odot \omega & =\left(\left(\left[\omega_{1}, \ldots, \omega_{n}\right] \circ p\right) \otimes \omega\right) \circ \rho^{-1} & \\
& =\left(\left[\omega_{1}, \ldots, \omega_{n}\right] \otimes \omega\right) \circ\left(\operatorname{instr}_{p} \otimes \mathrm{id}\right) \circ \rho^{-1} & \text { by Lemma 7.1 (1) } \\
& =\left(\left[\omega_{1}, \ldots, \omega_{n}\right] \otimes \omega\right) \circ \theta_{1}^{-1} \circ \operatorname{instr}_{\pi_{1}^{*}(p)} \circ \rho^{-1} & \text { by (10.4) } \\
& =\left[\omega_{1} \otimes \omega, \ldots, \omega_{n} \otimes \omega\right] \circ n \cdot \rho \circ \operatorname{instr}_{p} & \\
& \quad \text { since } \pi_{1}=\rho:(n \cdot 1) \otimes 1 \rightarrow n \cdot 1 \text { is pure by Assumption [3 (3) } \\
= & {\left[\omega_{1} \odot \omega, \ldots, \omega_{n} \odot \omega\right] \circ p} & \text { by Lemma 7.1 (1) again } \\
= & \emptyset_{i} p_{i}\left(\omega_{i} \odot \omega\right) .
\end{array}
$$

For the fourth point consider a 'weakened' predicate $\left(\pi_{1}\right)^{*}(p)=p \circ \pi_{1}: X \otimes Y \rightarrow X \rightarrow 1+1$. We use that $\kappa_{1}: 1 \rightarrow 1+1$ is the neutral element of $m$, see (the proof of) Proposition 10.2 (1) in:

$$
\begin{aligned}
p \odot 1=m \circ(p \otimes 1) & =m \circ\left(\mathrm{id} \otimes \kappa_{1}\right) \circ\left(p \otimes !_{Y}\right) \\
& =\rho \circ(p \otimes \mathrm{id}) \circ\left(\mathrm{id} \otimes !_{Y}\right) \\
& =p \circ \rho \circ\left(\mathrm{id} \otimes !_{Y}\right) \\
& =p \circ \pi_{1} \\
& =\left(\pi_{1}\right)^{*}(p) .
\end{aligned}
$$

Finally, we show that $(-) \odot p: \operatorname{Pred}(X) \rightarrow \operatorname{Pred}(X \otimes Y)$ preserves sums and scalar multiplication. Preservation of falsum is easy, since $0=\kappa_{2}: 1 \rightarrow 2$ is zero element for $m$, see Proposition 10.2 (11):

$$
0 \odot p=m \circ\left(\left(\kappa_{2} \circ !_{Y}\right) \otimes p\right)=\kappa_{2} \circ !_{2 \otimes 1} \circ\left(!_{X} \otimes p\right)=\kappa_{2} \circ !_{X \otimes Y}=0 .
$$

Next, if $q_{1} \perp q_{2}$, for $q_{1}, q_{2}: X \rightarrow 1+1$, via bound $b: X \rightarrow(1+1)+1$, then we construct a bound $c$ on $X \otimes Y$ via the east-south-west composite in:

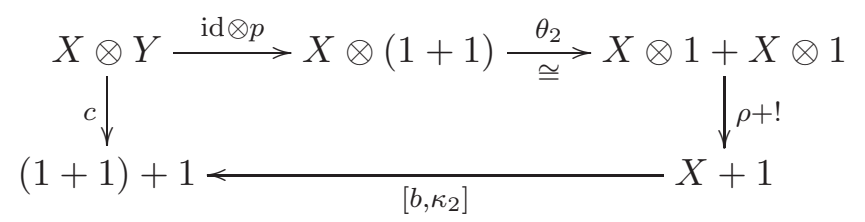

This bound $c$ proves $q_{1} \odot p \perp q_{2} \odot p$ and $\left(q_{1} \odot p\right) \oplus\left(q_{2} \odot p\right)=\left(q_{1} \otimes q_{2}\right) \odot p$. Preservation of scalar multiplication, and $1 \odot 1=1$ are left to the reader.

Examples 10.8. We briefly review the above pairing operation $\odot$ in the probabilistic and quantum case. We will see that in both cases $\odot$ is multiplication.

(1) In the Kleisli category $\mathcal{K} \ell(\mathcal{D})$ of the distribution monad $\mathcal{D}$ on Sets the pairing $\varphi \odot$ $\psi \in \mathcal{D}(X \times Y)$ of two states/distributions $\varphi \in \mathcal{D}(X)$ and $\psi \in \mathcal{D}(Y)$ is given by their product:

$$
(\varphi \odot \psi)(x, y)=\operatorname{dst}(\varphi, \psi)(x, y)=\varphi(x) \cdot \psi(y) .
$$

where dst is the 'double-strength' map from Appendix A.1 The pairing $p \odot q \in$ $[0,1]^{X \times Y}$ of predicates $p \in[0,1]^{X}$ and $q \in[0,1]^{Y}$ is obtained similarly as:

$$
(p \odot q)(x, y)=p(x) \cdot q(y) \text {. }
$$


Thus, indeed, as in Lemma 10.7 (1),

$$
\begin{aligned}
\varphi \odot \psi \models p \odot q & =\sum_{x, y}(\varphi \odot \psi)(x, y) \cdot(p \odot q)(x, y) \\
& =\sum_{x, y} \varphi(x) \cdot \psi(y) \cdot p(x) \cdot q(y) \\
& =\left(\sum_{x} \varphi(x) \cdot p(x)\right) \cdot\left(\sum_{y} \psi(y) \cdot q(y)\right) \\
& =(\varphi \models p) \cdot(\psi=q) .
\end{aligned}
$$

(2) For two $C^{*}$-algebras $A, B$ with effects $e \in[0,1]_{A}, d \in[0,1]_{B}$ we have the pairing effect $e \odot d=e \otimes d \in A \otimes B$. It is obtained as follows. The effect $e \in[0,1]_{A}$ corresponds to the positive unital map $f_{e}: \mathbb{C}^{2} \rightarrow A$ given by $f_{e}(|0\rangle)=e$ and $f_{e}(|1\rangle)=1-e$, and similarly for $d$. The function $f_{e \odot d}: \mathbb{C}^{2} \rightarrow A \otimes B$ is then the composite $f_{e \odot d}=\left(f_{e} \otimes f_{d}\right) \circ m$, with multiplication $m$ from (10.7). Thus:

$$
\begin{aligned}
e \odot d=f_{e \odot d}(|0\rangle)=\left(f_{e} \otimes f_{d}\right)(m(|0\rangle)) & =\left(f_{e} \otimes f_{d}\right)(|0\rangle \otimes|0\rangle) \quad \text { by (10.7) } \\
& =f_{e}(|0\rangle) \otimes f_{d}(|0\rangle) \\
& =e \otimes d .
\end{aligned}
$$

Point (2) in Lemma 10.7 says that there is a retraction $\operatorname{Stat}(X) \times \operatorname{Stat}(Y) \longmapsto \operatorname{Stat}(X \otimes Y)$, since first pairing and then projecting gives the original output. In the category of sets the retraction is an isomorphism, but in general this is not the case. There is no such isomorphism because of both entanglement and dependence in the quantum world, and because of dependence in the probabilistic world. We briefly illustrate this fundamental phenomenon in the current setting.

Examples 10.9 (Dependence and entanglement). In a probabilistic setting as given by the category $\mathcal{K} \ell(\mathcal{D})$, consider a state on the tensor product $X \times Y$ of two sets $X, Y$. It is a discrete probability distribution $\varphi \in \mathcal{D}(X \times Y)$ on $X$ and $Y$. Because it describes probabilities on two sets, such a $\varphi$ is often called a joint distribution. One can take its two marginals $\left(\pi_{1}\right)_{*}(\varphi)=\mathcal{D}\left(\pi_{1}\right)(\varphi) \in \mathcal{D}(X)$ and $\left(\pi_{2}\right)_{*}(\varphi)=\mathcal{D}\left(\pi_{2}\right)(\varphi) \in \mathcal{D}(Y)$. If we now pair these marginals we may ask if the original joint distribution re-appears, that is if:

$$
\left(\pi_{1}\right)_{*}(\varphi) \odot\left(\pi_{2}\right)_{*}(\varphi) \stackrel{?}{=} \varphi .
$$

But this equation precisely expresses that $\varphi$ is a product distribution (i.e. is factorisable). Hence the equation does not always hold.

Similarly, in the quantum world, a state $\omega: A \otimes B \rightarrow \mathbb{C}$ can, in general, not be reconstructed from its two marginals $\left(\pi_{1}\right)_{*}(\omega)=\omega \circ \kappa_{1}: A \rightarrow \mathbb{C}$ and $\left(\pi_{2}\right)_{*}(\omega)=\omega \circ \kappa_{2}: B \rightarrow \mathbb{C}$, since in general we do not have $\left(\pi_{1}\right)_{*}(\omega) \odot\left(\pi_{2}\right)_{*}(\omega)=\omega$.

Here is concrete qubit example, for $A=B=\mathcal{B}\left(\mathbb{C}^{2}\right)$. We consider the non-entangled vector $|u\rangle=|00\rangle=|0\rangle \otimes|0\rangle \in \mathbb{C}^{2} \otimes \mathbb{C}^{2}$ and also the entangled (EPR) vector $|v\rangle=$ $\frac{1}{\sqrt{2}}(|00\rangle+|11\rangle) \in \mathbb{C}^{2} \otimes \mathbb{C}^{2}$. There are associated states $\omega_{u}, \omega_{v}: \mathcal{B}\left(\mathbb{C}^{2} \otimes \mathbb{C}^{2}\right) \rightarrow \mathbb{C}$, given by $\omega_{u}(f)=\langle u|f| u\rangle$ and $\omega_{v}(f)=\langle v|f| v\rangle$. The marginals are given by $\left(\pi_{1}\right)_{*}\left(\omega_{u}\right)(M)=$ $\omega_{u}(M \otimes$ id $)$ and $\left(\pi_{2}\right)_{*}\left(\omega_{u}\right)(N)=\omega_{u}(\mathrm{id} \otimes N)$, and similarly for $v$. The (non-entangled) state 
$\omega_{u}$ can be reconstructed via pairing from its marginals:

$$
\begin{aligned}
\left(\left(\pi_{1}\right)_{*}\left(\omega_{u}\right) \odot\left(\pi_{2}\right)_{*}\left(\omega_{u}\right)\right)(M \otimes N) & =\left(\pi_{1}\right)_{*}\left(\omega_{u}\right)(M) \cdot\left(\pi_{2}\right)_{*}\left(\omega_{u}\right)(N) \\
& =\omega_{u}(M \otimes \mathrm{id}) \cdot \omega_{u}(\mathrm{id} \otimes N) \\
& =\langle 00|(M \otimes \mathrm{id})| 00\rangle \cdot\langle 00|(\mathrm{id} \otimes N)| 00\rangle \\
& =(\langle 0|M| 0\rangle \cdot\langle 0 \mid 0\rangle) \cdot(\langle 0 \mid 0\rangle \cdot\langle 0|N| 0\rangle) \\
& =\langle 0|M| 0\rangle \cdot\langle 0|N| 0\rangle \\
& =\langle 00|(M \otimes N)| 00\rangle \\
& =\omega_{u}(M \otimes N) .
\end{aligned}
$$

Because $|v\rangle=\frac{1}{\sqrt{2}}(|00\rangle+|11\rangle)$ is an entangled state, we do not have such an equation for $v$. We step through the computation to indicate where this fails:

$$
\begin{aligned}
& \left(\left(\pi_{1}\right)_{*}\left(\omega_{v}\right) \odot\left(\pi_{2}\right)_{*}\left(\omega_{v}\right)\right)(M \otimes N) \\
& =\omega_{v}(M \otimes \mathrm{id}) \cdot \omega_{v}(\mathrm{id} \otimes N) \\
& =\frac{1}{2}\left(\left\langle00|+\langle 11|)(M \otimes \mathrm{id})(|00\rangle+|11\rangle) \cdot \frac{1}{2}(\langle 00|+\langle 11|)(\mathrm{id} \otimes N)(|00\rangle+|11\rangle)\right.\right. \\
& =\frac{1}{4}(\langle 0|M| 0\rangle+\langle 1|M| 1\rangle) \cdot(\langle 0|N| 0\rangle+\langle 1|N| 1\rangle) \quad \text { since }\langle 0 \mid 1\rangle=\langle 1 \mid 0\rangle=0 \\
& =\frac{1}{4}(\langle 0|M| 0\rangle \cdot\langle 0|N| 0\rangle+\langle 0|M| 0\rangle \cdot\langle 1|N| 1\rangle+\langle 1|M| 1\rangle \cdot\langle 0|N| 0\rangle+\langle 1|M| 1\rangle \cdot\langle 1|N| 1\rangle) \\
& \neq \frac{1}{2}(\langle 0|M| 0\rangle \cdot\langle 0|N| 0\rangle+\langle 0|M| 1\rangle \cdot\langle 0|N| 1\rangle+\langle 1|M| 0\rangle \cdot\langle 1|N| 0\rangle+\langle 1|M| 1\rangle \cdot\langle 1|N| 1\rangle) \\
& =\frac{1}{2}(\langle 00|(M \otimes N)| 00\rangle+\langle 00|(M \otimes N)| 11\rangle+\langle 11|(M \otimes N)| 00\rangle+\langle 11|(M \otimes N)| 11\rangle) \\
& =\frac{1}{2}(\langle 00|+\langle 11|)(M \otimes N)(|00\rangle+|11\rangle) \\
& =\omega_{v}(M \otimes N) .
\end{aligned}
$$

In the end we mention that the distribution of the tensor $\otimes$ over coproducts + is useful to interpret operations in context. For instance, the guarded command $p ?\left[f_{1}, \ldots, f_{n}\right]$ from (7.7) can now be interpreted more generally: given an $n$-test $p: X \rightarrow n \cdot 1$, and $n$ maps $f_{i}: X \otimes Z \rightarrow$ $Y$ with an additional context parameter $Z$ we can interpret the test map $p ?\left[f_{1}, \ldots, f_{n}\right]$ formally via weakening as $\pi_{1}^{*}(p) ?\left[f_{1}, \ldots, f_{n}\right]$ in:

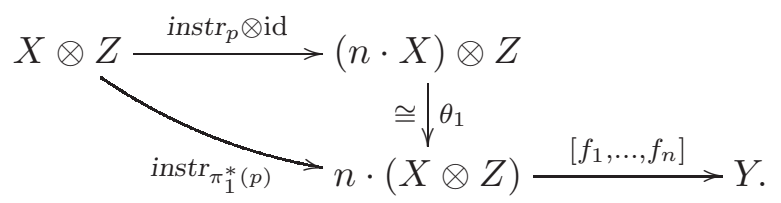

The reasoning rule from Lemma 9.6 still applies, with weakened tests $\pi_{1}^{*}(p)$. As special case we have an equation, for maps $h_{i}: X \rightarrow Y$,

$$
p ?\left[h_{1}, \ldots, h_{n}\right] \otimes \mathrm{id}=\pi_{1}^{*}(p) ?\left[h_{1} \otimes \mathrm{id}, \ldots, h_{n} \otimes \mathrm{id}\right] .
$$

\section{Quantum states}

In this section we add our final assumption about the existence of a "quantum object". We use it in a subsequent quantum protocol. 
Assumption 4. Let $\mathbf{B}$ be a category satisfying Assumption 3. This category contains a special object $Q \in \mathbf{B}$ with two states and a predicate:

$$
1 \stackrel{\uparrow}{\longrightarrow} Q \quad 1 \stackrel{\downarrow}{\longrightarrow} Q \quad Q \stackrel{\text { isup }}{\longrightarrow} 1+1
$$

such that the following diagram commutes,

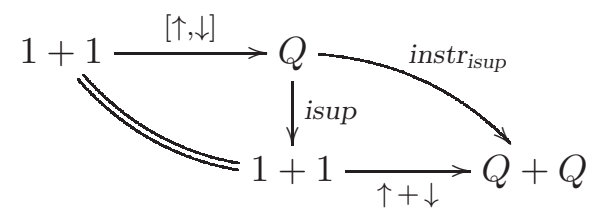

and the predicate isup is not side-effect-free, or equivalently, the map isup: $Q \rightarrow 1+1$ is not an isomorphism.

In general, the object $2=1+1$ comes equipped with two states, namely $1=\kappa_{1}: 1 \rightarrow 2$ and $0=\kappa_{2}: 1 \rightarrow 2$. The identity map $2 \rightarrow 1+1$ can be understood as a 'isup' predicate. In this way we obtain classical states, a bit like the object $Q$ in the above assumption. But the object $Q$ is different, since it is explicitly required that there is not an isomorphism $2 \cong Q$.

The states $\uparrow, \downarrow$ correspond to the operations $n e w_{0}, n e w_{1}$ in 75 , and the predicate isup is called measure there. The above description focuses on the states and measurement operations, in line with the rest of the paper. The unitary operations for state transformations are clearly missing. They do play an important role in quantum programming language, see [74, 75, 82].

Example 11.1. In the opposite categories $\left(\mathbf{C s t a r}_{\mathrm{PU}}\right)^{\text {op }}$ and $\left(\mathbf{C s t a r}_{\mathrm{CPU}}\right)^{\text {op }}$ of $C^{*}$-algebras with (completely) positive unital maps we can take $Q=\mathcal{B}\left(\mathbb{C}^{2}\right)=M_{2}(\mathbb{C})$. Let's write an element $M \in Q$ as a $2 \times 2$ matrix:

$$
M=\left(\begin{array}{ll}
M_{00} & M_{01} \\
M_{10} & M_{11}
\end{array}\right) \quad \text { with matrix entries } \quad M_{i j} \in \mathbb{C} .
$$

Then we can define two states $\uparrow, \downarrow$ as (completely) positive unital maps $Q \rightarrow \mathbb{C}$ via:

$$
\uparrow(M)=M_{00}=\langle 0|M| 0\rangle \quad \text { and } \quad \downarrow(M)=M_{11}=\langle 1|M| 1\rangle .
$$

As predicate isup: $\mathbb{C}^{2} \rightarrow Q$ we define:

$$
\operatorname{isup}(z, w)=z|0\rangle\langle 0|+w| 1\rangle\langle 1|=\left(\begin{array}{cc}
z & 0 \\
0 & w
\end{array}\right) .
$$

It corresponds to the effect isup $(1,0)=\left(\begin{array}{ll}1 & 0 \\ 0 & 0\end{array}\right)=|0\rangle\langle 0|$. This effect is obviously a projection, with orthocomplement $(|0\rangle\langle 0|)^{\perp}=|1\rangle\langle 1|$. The associated instrument instr isup $_{\text {in }}: Q \oplus Q \rightarrow Q$ is thus:

$$
\operatorname{instr}_{\text {isup }}(M, N)=|0\rangle\langle 0|M| 0\rangle\langle 0|+| 1\rangle\langle 1|N| 1\rangle\langle 1|=\left(\begin{array}{cc}
M_{00} & 0 \\
0 & N_{11}
\end{array}\right) .
$$

It is easy to see that Diagram (11.1) commutes:

$$
\begin{aligned}
(\langle\uparrow, \downarrow\rangle \circ \operatorname{isup})(z, w) & =\left(\uparrow\left(\begin{array}{cc}
z & 0 \\
0 & w
\end{array}\right), \downarrow\left(\begin{array}{cc}
z & 0 \\
0 & w
\end{array}\right)\right)=(z, w) \\
(\operatorname{isup} \circ(\uparrow \oplus \downarrow))(M, N) & =\operatorname{isup}\left(M_{00}, N_{11}\right)=\left(\begin{array}{cc}
M_{00} & 0 \\
0 & N_{11}
\end{array}\right)=\operatorname{instr}_{\text {isup }}(M, N) .
\end{aligned}
$$




\section{Remark 11.2.}

(1) In the opposite $\mathbf{R n g}$ op of the category of rings — not necessarily commutative - we can use the object $Q=M_{2}(\mathbb{Z})$ of $2 \times 2$ matrices over the integers, with similar states and predicate. (In this ring example one does have the structure described Assumption 4 , but Assumption 3 is not satisfied.)

(2) Via the isomorphisms $\operatorname{Stat}\left(\mathcal{B}\left(\mathbb{C}^{2}\right)\right) \cong \mathcal{D M}\left(\mathbb{C}^{2}\right)$ from $(5.3)$ the state $\uparrow$ in Example 11.1 corresponds to the density matrix $|0\rangle\langle 0| \in \mathcal{D M}\left(\mathbb{C}^{2}\right)$, since for $M \in \mathcal{B}\left(\mathbb{C}^{2}\right)$,

$$
\operatorname{tr}(|0\rangle\langle 0| M)=\langle 0|M| 0\rangle=\uparrow(M) .
$$

If we have a program $f: Q \otimes X \rightarrow Y$ we can insert a state like $\uparrow$ or $\downarrow$ in the first component. Category-theoretically this involves taking the composite:

$$
X \cong 1 \otimes X \stackrel{\uparrow \otimes \mathrm{id}}{\longrightarrow} Q \otimes X \stackrel{f}{\longrightarrow} Y
$$

In the language of [75] this operation would be written as new $(v \cdot f(v,-))$. More informally we shall write it below as: let $v=\uparrow$ in $f(v \otimes-)$. We then have:

$$
\left(\omega \models(\text { let } v=\uparrow \text { in } f(v \otimes-))^{*}(q)\right)=\left(\uparrow \odot \omega \models f^{*}(q)\right) .
$$

We conclude with a description of a familiar protocol in the setting developed in this article.

Example 11.3 (Superdense coding). In the superdense coding protocol Alice sends two classical bits to Bob by transferring her part of a shared, entangled quantum state. In a category with a quantum object $Q$ as in Assumption 4 this protocol can be described as a map sdc: $4 \rightarrow 4$ consisting of three consecutive steps:

$$
s d c=\left(4 \stackrel{\text { init }}{\longrightarrow} 4 \otimes Q \otimes Q \stackrel{t_{A} \otimes \mathrm{id}}{\longrightarrow} Q \otimes Q \stackrel{t_{B}}{\longrightarrow} 4\right)
$$

The correctness of the protocol means that this composite is the identity map.

We shall describe this sdc map (11.2) in greater detail in the category $\left(\mathbf{C s t a r}_{\mathrm{CPU}}\right)^{\mathrm{op}}$, with $Q=\mathcal{B}\left(\mathbb{C}^{2}\right)$ as in Example11.1. In pseudo-code this protocol sdc: $\mathbb{C}^{4} \rightarrow \mathbb{C}^{4}$ is described in Figure 4. It is not our aim to develop the syntax of this code fragment in detail. Instead we explain its interpretation in the category $\left(\mathbf{C s t a r}_{\mathrm{CPU}}\right)^{\mathrm{op}}$. For the two test operations we use the meaning described in (17.6).

We start by recalling some basic material. The Bell basis of $\mathbb{C}^{4}$ is given by the four vectors:

$$
\begin{array}{ll}
\left|b_{1}\right\rangle=\frac{1}{\sqrt{2}}(|00\rangle+|11\rangle) & \left|b_{2}\right\rangle=\frac{1}{\sqrt{2}}(|01\rangle+|10\rangle) \\
\left|b_{3}\right\rangle=\frac{1}{\sqrt{2}}(|00\rangle-|11\rangle) & \left|b_{4}\right\rangle=\frac{1}{\sqrt{2}}(|01\rangle-|10\rangle)
\end{array}
$$

The associated projections $e_{i}=\left|b_{i}\right\rangle\left\langle b_{i}\right| \in \mathcal{B}\left(\mathbb{C}^{4}\right) \cong \mathcal{B}\left(\mathbb{C}^{2}\right) \otimes \mathcal{B}\left(\mathbb{C}^{2}\right)=Q \otimes Q$ are described explicitly by the four matrices:

$$
e_{1}=\frac{1}{2}\left(\begin{array}{llll}
1 & 0 & 0 & 1 \\
0 & 0 & 0 & 0 \\
0 & 0 & 0 & 0 \\
1 & 0 & 0 & 1
\end{array}\right) \quad e_{2}=\frac{1}{2}\left(\begin{array}{llll}
0 & 0 & 0 & 0 \\
0 & 1 & 1 & 0 \\
0 & 1 & 1 & 0 \\
0 & 0 & 0 & 0
\end{array}\right) \quad e_{3}=\frac{1}{2}\left(\begin{array}{cccc}
1 & 0 & 0 & -1 \\
0 & 0 & 0 & 0 \\
0 & 0 & 0 & 0 \\
-1 & 0 & 0 & 1
\end{array}\right) \quad e_{4}=\frac{1}{2}\left(\begin{array}{cccc}
0 & 0 & 0 & 0 \\
0 & 1 & -1 & 0 \\
0 & -1 & 1 & 0 \\
0 & 0 & 0 & 0
\end{array}\right)
$$

They satisfy $e_{1} \otimes e_{2} \otimes e_{3} \otimes e_{4}=$ id and thus form a 4-test $\mathbb{C}^{4} \rightarrow Q \otimes Q$, as used in Bob's test in Figure 4. Since $e_{i}^{2}=e_{i}$ we have $\sqrt{e_{i}}=e_{i}$. The associated instrument $(Q \otimes Q)^{4} \rightarrow Q \otimes Q$ thus sends $\left(x_{1}, x_{2}, x_{3}, x_{4}\right)$ to $\sum_{i} e_{i} \cdot x_{i} \cdot e_{i}$. 
The first element $\left|b_{1}\right\rangle=\frac{1}{\sqrt{2}}(|00\rangle+|11\rangle) \in Q \otimes Q$ of the Bell basis can be obtained from the base vector $|00\rangle=\uparrow \otimes \uparrow \in Q \otimes Q$ by first applying $H \otimes$ id — where $H$ is Hadamard and then conditional negation CNOT. This is done in the initialisation fragment in Figure 4, The resulting initialisation map from (11.2) has type init: $\mathbb{C}^{4} \otimes Q \otimes Q \rightarrow \mathbb{C}^{4}$ in Cstar $_{\mathrm{CPU}}$ and is given by $\operatorname{init}(\vec{z} \otimes x)=\left\langle b_{1}|x| b_{1}\right\rangle \cdot \vec{z}$.

For Alice's test we need the four Pauli matrices in $\mathcal{B}\left(\mathbb{C}^{2}\right)$ :

$$
\sigma_{1}=\left(\begin{array}{ll}
1 & 0 \\
0 & 1
\end{array}\right) \quad \sigma_{2}=X=\left(\begin{array}{ll}
0 & 1 \\
1 & 0
\end{array}\right) \quad \sigma_{3}=Z=\left(\begin{array}{cc}
1 & 0 \\
0 & -1
\end{array}\right) \quad \sigma_{4}=X Z=\left(\begin{array}{cc}
0 & -1 \\
1 & 0
\end{array}\right) .
$$

When we apply $\sigma_{i} \otimes$ id to the Bell basis vectors $\left|b_{j}\right\rangle$ we see that:

$$
\begin{cases}\sigma_{1} \otimes \mathrm{id}=\mathrm{id} & \text { changes nothing } \\ \sigma_{2} \otimes \mathrm{id} & \text { exchanges }\left|b_{1}\right\rangle,\left|b_{2}\right\rangle \text { and }\left|b_{3}\right\rangle,\left|b_{4}\right\rangle \\ \sigma_{3} \otimes \mathrm{id} & \text { exchanges }\left|b_{1}\right\rangle,\left|b_{3}\right\rangle \text { and }\left|b_{2}\right\rangle,\left|b_{4}\right\rangle \\ \sigma_{4} \otimes \mathrm{id} & \text { exchanges }\left|b_{1}\right\rangle,\left|b_{4}\right\rangle \text { and }\left|b_{2}\right\rangle,\left|b_{3}\right\rangle .\end{cases}
$$

Alice's test map $t_{A}: Q \otimes Q \rightarrow \mathbb{C}^{4} \otimes Q \otimes Q$ in $\operatorname{Cstar}_{\mathrm{CPU}}$ is given by $t_{A}(x)=\sum_{i}|i\rangle \otimes$ $\mathcal{B}\left(\sigma_{i} \otimes \mathrm{id}\right)(x)$, where $|i\rangle \in \mathbb{C}^{4}$ is a standard base vector.

Finally, the test operation performed by Bob in Figure 4 is the map $t_{B}: \mathbb{C}^{4} \rightarrow Q \otimes Q$ given by $t_{B}(\vec{z})=\sum_{i} z_{i} \cdot e_{i}$. Now we are ready to calculate the composite (11.2), in reverse order:

$$
\begin{aligned}
\operatorname{sdc}\left(z_{1}, z_{2}, z_{3}, z_{4}\right) & =\left(\text { init } \circ t_{A} \circ t_{B}\right)\left(z_{1}, z_{2}, z_{3}, z_{4}\right) \\
& =\left(\text { init } \circ t_{A}\right)\left(\sum_{i} z_{i} \cdot e_{i}\right) \\
& =\operatorname{init}\left(\sum_{j}|j\rangle \otimes \mathcal{B}\left(\sigma_{j} \otimes \mathrm{id}\right)\left(\sum_{i} z_{i} \cdot e_{i}\right)\right) \\
& =\operatorname{init}\left(\sum_{i, j} z_{i} \cdot|j\rangle \otimes\left(\sigma_{j} \otimes \mathrm{id}\right)^{\dagger}\left|b_{i}\right\rangle\left\langle b_{i}\right|\left(\sigma_{j} \otimes \mathrm{id}\right)\right) \\
& =\sum_{i, j} z_{i} \cdot\left\langle b_{1}\left|\left(\sigma_{j} \otimes \mathrm{id}\right)^{\dagger}\right| b_{i}\right\rangle\left\langle b_{i}\left|\left(\sigma_{j} \otimes \mathrm{id}\right)\right| b_{1}\right\rangle|j\rangle \\
& =\sum_{j} z_{j} \cdot|j\rangle \quad \text { by (11.3) } \\
& =\left(z_{1}, z_{2}, z_{3}, z_{4}\right) .
\end{aligned}
$$

It remains a challenge to prove the correctness of protocols like this via the dynamic logic that is sketched in this paper. Such a challenge requires a further syntactical and logical development of the logic, see [4], which is beyond the scope of the current paper.

\section{Conclusions And Further WORK}

This paper develops a quantitative categorical logic via four assumptions, starting from predicates as maps $X \rightarrow 1+1$ and states as maps $1 \rightarrow X$, in a dual relationship. It involves abstract forms of validity, predicate- and state-transformers, measurement instruments, and test programs \& predicates. The theory applies to standard set-theoretic, probabilistic, and quantum models.

The first version of the article appeared online in 2012. Since then the research has been extended in several other publications. In particular, 4 introduces a predicate logic based on effects, 47] elaborates the role of coproduct-preservation by the states functor in a state-and-effect triangle (5.4), [17] describes how instruments (from Section 7 arise in many examples from quotients and comprehension in the logic, [16] gives an equivalent description of the notion of effectus as a category of partial maps, and [43] describes many 


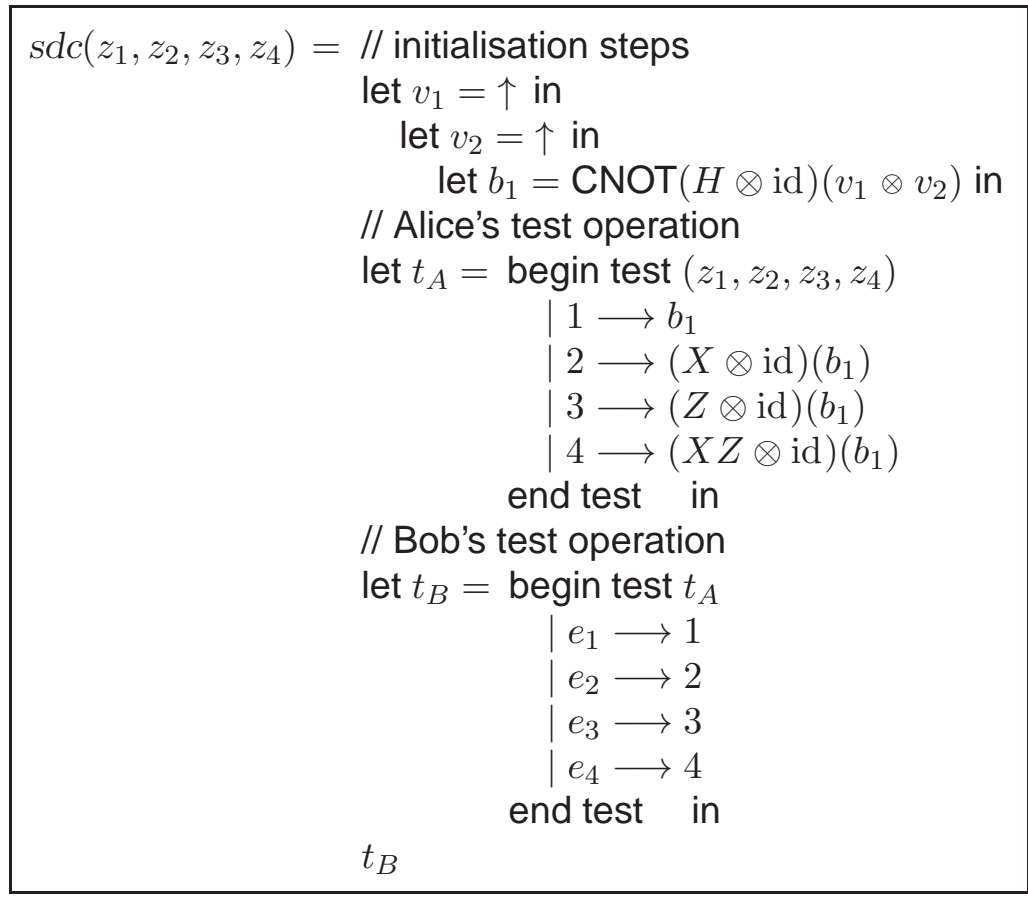

Figure 4: Pseudo code for the superdense coding function sdc from Example 11.3.

state-and-effect triangle examples. There is more as yet unpublished work, especially giving characterisations of 'Boolean' and 'classical' (probabilistic) effectuses.

Acknowledgements. The work presented in this article acquired its current shape during a period of roughly three years of research, discussion, revision and extension. The main sparring partner was Bas Westerbaan to whom I owe a lot. Other colleagues that contributed with comments and feedback include Robin Adams, Kenta Cho, Tobias Fritz, Robert Furber, Ichiro Hasuo, Chris Heunen, Jorik Mandemaker, Frank Roumen, Sam Staton and Bram Westerbaan. I thank them all, and also the anonymous referees for their constructive comments.

\section{REFERENCES}

[1] S. Abramsky. No-cloning in categorical quantum mechanics. In S. Gay and I. Mackie, editors, Semantical Techniques in Quantum Computation, pages 1-28. Cambridge Univ. Press, 2010.

[2] S. Abramsky and B. Coecke. A categorical semantics of quantum protocols. In Logic in Computer Science, pages 415-425. IEEE, Computer Science Press, 2004.

[3] S. Abramsky and B. Coecke. A categorical semantics of quantum protocols. In K. Engesser, Dov M. Gabbai, and D. Lehmann, editors, Handbook of Quantum Logic and Quantum Structures: Quantum Logic, pages 261-323. North Holland, Elsevier, Computer Science Press, 2009.

[4] R. Adams. QPEL: Quantum program and effect language. In B. Coecke, I. Hasuo, and P. Panangaden, editors, Quantum Physics and Logic (QPL) 2014, number 172 in Elect. Proc. in Theor. Comp. Sci., pages 133-153, 2014.

[5] M. Arbib and E. Manes. Algebraic Approaches to Program Semantics. Texts and Monogr. in Comp. Sci. Springer, Berlin, 1986.

[6] W. Arveson. An Invitation to $C^{*}$-Algebra. Springer-Verlag, 1981. 
[7] S. Awodey. Category Theory. Oxford Logic Guides. Oxford Univ. Press, 2006.

[8] G. Birkhoff and J. von Neumann. The logic of quantum mechanics. Ann. Math., 37:823-843, 1936.

[9] F. Borceux. Handbook of Categorical Algebra, volume 50, 51 and 52 of Encyclopedia of Mathematics. Cambridge Univ. Press, 1994.

[10] P. Busch. Effect. In D. Greenberger, K. Hentschel, and F. Weinert, editors, Compendium of Quantum Physics, pages 179-180. Springer, 2009.

[11] P. Busch, P. Lahti, and P. Mittelstaedt. The Quantum Theory of Measurement. Springer, $2^{\text {nd }}$ edition, 1996.

[12] P. Busch and J. Singh. Lüders theorem for unsharp quantum measurements. Phys. Letters A, 249:10-12, 1998.

[13] A. Carboni, S. Lack, and R. Walters. Introduction to extensive and distributive categories. Journ. of Pure Es Appl. Algebra, 84(2):145-158, 1993.

[14] C. Chang. Algebraic analysis of many-valued logics. Trans. Amer. Math. Soc., 88:476-490, 1958.

[15] K. Cho. Semantics for a quantum programming language by operator algebras. In B. Coecke, I. Hasuo, and P. Panangaden, editors, Quantum Physics and Logic (QPL) 2014, number 172 in Elect. Proc. in Theor. Comp. Sci., pages 165-190, 2014.

[16] K. Cho. Total and partial computation in categorical quantum foundations. In C. Heunen en J. Vicary, editor, Quantum Physics and Logic (QPL) 2015, Elect. Proc. in Theor. Comp. Sci., 2015.

[17] K. Cho, B. Jacobs, A. Westerbaan, and B. Westerbaan. Quotient comprehension chains. In C. Heunen en J. Vicary, editor, Quantum Physics and Logic (QPL) 2015, Elect. Proc. in Theor. Comp. Sci., 2015.

[18] F. Chovanec and F. Kôpka. D-posets. In K. Engesser, Dov M. Gabbai, and D. Lehmann, editors, Handbook of Quantum Logic and Quantum Structures: Quantum Structures, pages 367-428. North Holland, Elsevier, Computer Science Press, 2007.

[19] B. Coecke and D. Pavlović. Quantum measurements without sums. In G. Chen, L. Kauffman, and S. Lamonaco, editors, Mathematics of Quantum Computing and Technology, pages 559-596. Taylor and Francis, 2008.

[20] E. Davies and J. Lewis. An operational approach to quantum probability. Communic. Math. Physics, 17:239-260, 1970.

[21] E. D'Hondt and P. Panangaden. Quantum weakest preconditions. Math. Struct. in Comp. Sci., 16(3):429-451, 2006.

[22] D. Dieks. Communication by EPR devices. Phys. Letters A, 92(6):271-272, 1982.

[23] E. Dijkstra. Guarded commands, nondeterminacy and formal derivation of programs. Communications of the ACM, 18(8):453-457, 1975.

[24] A. Dvurečenskij and S. Pulmannová. New Trends in Quantum Structures. Kluwer Acad. Publ., Dordrecht, 2000.

[25] D. J. Foulis and M.K. Bennett. Effect algebras and unsharp quantum logics. Found. Physics, 24(10):1331-1352, 1994.

[26] R. Furber and B. Jacobs. From Kleisli categories to commutative $C^{*}$-algebras: Probabilistic Gelfand duality. In R. Heckel and S. Milius, editors, Conference on Algebra and Coalgebra in Computer Science (CALCO 2013), number 8089 in Lect. Notes Comp. Sci., pages 141-157. Springer, Berlin, 2013.

[27] R. Furber and B. Jacobs. Towards a categorical account of conditional probability, 2013. QPL 2013, see arxiv.org/abs/1306.0831.

[28] M. Giry. A categorical approach to probability theory. In B. Banaschewski, editor, Categorical Aspects of Topology and Analysis, number 915 in Lect. Notes Math., pages 68-85. Springer, Berlin, 1982.

[29] R. Giuntini and H. Greuling. Toward a formal language for unsharp properties. Found. Physics, 19:769$780,1994$.

[30] J. Goguen and R. Burstall. Institutions: Abstract model theory for specification and programming. Journ. ACM, 39(1):95-146, 1992.

[31] S. Gudder and R. Greechie. Sequential products on effect algebras. Reports on Math. Physics, 49(1):87$111,2002$.

[32] S. Gudder and G. Nagy. Sequential quantum measurements. Journ. Math. Physics, 42:5212-5222, 2001.

[33] E. Haghverdi. Unique decomposition categories, geometry of interaction and combinatory logic. Math. Struct. in Comp. Sci., 10:205-231, 2000.

[34] D Harel, D. Kozen, and J. Tiuryn. Dynamic Logic. MIT Press, Cambridge, MA, 2000. 
[35] T. Heinosaari and M. Ziman. The Mathematical Language of Quantum Theory. From Uncertainty to Entanglement. Cambridge Univ. Press, 2012.

[36] B. Jacobs. Semantics of weakening and contraction. Ann. Pure \& Appl. Logic, 69(1):73-106, 1994.

[37] B. Jacobs. Categorical Logic and Type Theory. North Holland, Amsterdam, 1999.

[38] B. Jacobs. Convexity, duality, and effects. In C. Calude and V. Sassone, editors, IFIP Theoretical Computer Science 2010, number 82(1) in IFIP Adv. in Inf. and Comm. Techn., pages 1-19. Springer, Boston, 2010.

[39] B. Jacobs. Probabilities, distribution monads, and convex categories. Theor. Comp. Sci., 412(28):33233336, 2011.

[40] B. Jacobs. Measurable spaces and their effect logic. In Logic in Computer Science. IEEE, Computer Science Press, 2013.

[41] B. Jacobs. On block structures in quantum computation. In D. Kozen and M. Mislove, editors, Math. Found. of Programming Semantics, volume 298 of Elect. Notes in Theor. Comp. Sci., pages 233-255. Elsevier, Amsterdam, 2013.

[42] B. Jacobs. Dijkstra and Hoare monads in monadic computation. TCS, 2015. DOI http://dx.doi.org/10.1016/j.tcs.2015.03.020.

[43] B. Jacobs. A recipe for state and effect triangles. In L. Moss and P. Sobocinski, editors, Conference on Algebra and Coalgebra in Computer Science (CALCO 2015), LIPIcs, 2015.

[44] B. Jacobs and J. Mandemaker. Coreflections in algebraic quantum logic. Found. of Physics, 42(7):932958, 2012.

[45] B. Jacobs and J. Mandemaker. The expectation monad in quantum foundations. In B. Jacobs, P. Selinger, and B. Spitters, editors, Quantum Physics and Logic (QPL) 2011, volume 95 of Elect. Proc. in Theor. Comp. Sci., pages 143-182, 2012.

[46] B. Jacobs and A. Westerbaan. An effect-theoretic account of Lebesgue integration, 2015. Math. Found. of Programming Semantics XXXI.

[47] B. Jacobs, B. Westerbaan, and A. Westerbaan. States of convex sets. In A. Pitts, editor, Foundations of Software Science and Computation Structures, number 9034 in Lect. Notes Comp. Sci., pages 87-101. Springer, Berlin, 2015.

[48] P. Johnstone. Stone Spaces. Number 3 in Cambridge Studies in Advanced Mathematics. Cambridge Univ. Press, 1982.

[49] R. Kadison. A representation theory for commutative topological algebra. Memoirs of the AMS, 7, 1951.

[50] G. Kalmbach. Orthomodular Lattices. Academic Press, London, 1983.

[51] F. Katrnoška. Logics of idempotents of rings. In C. Bandt, J. Flachsmeyer, and H. Haase, editors, Topology, Measure, and Fractals, number 66 in Math. Research, pages 131-136. Academie Verlag, 1992.

[52] M. Kelly and M. Laplaza. Coherence for compact closed categories. Journ. of Pure ES Appl. Algebra, 19:193-213, 1980.

[53] A. Kock. Bilinearity and cartesian closed monads. Math. Scand., 29:161-174, 1971.

[54] A. Kock. Closed categories generated by commutative monads. Journ. Austr. Math. Soc., XII:405-424, 1971.

[55] D. Kozen. Semantics of probabilistic programs. Journ. Comp. Syst. Sci, 22(3):328-350, 1981.

[56] D. Kozen. A probabilistic PDL. Journ. Comp. Syst. Sci, 30(2):162-178, 1985.

[57] K. Kraus. States, Effects, and Operations. Springer Verlag, Berlin, 1983.

[58] N.P. Landsman. Algebraic quantum mechanics. In D. Greenberger, K. Hentschel, and F. Weinert, editors, Compendium of Quantum Physics, pages 6-10. Springer, 2009.

[59] P. Martin-Löf. Intuitionistic Type Theory. Bibliopolis, Napoli, 1984.

[60] S. Mac Lane. Categories for the Working Mathematician. Springer, Berlin, 1971.

[61] S. Mac Lane and I. Moerdijk. Sheaves in Geometry and Logic. A First Introduction to Topos Theory. Springer, New York, 1992.

[62] E. Moggi. Notions of computation and monads. Inf. \& Comp., 93(1):55-92, 1991.

[63] D. Mundici. Advanced Eukasiewicz calculus and MV-algebras, volume 35 of Trends in Logic. Springer, 2011.

[64] J. von Neumann. Mathematische Grundlagen der Quantenmechanik. Springer, Berlin, 1932.

[65] B. Nordström, K. Peterson, and J.M. Smith. Programming in Martin-Löf's Type Theory: an introduction. Number 7 in Logic Guides. Oxford Science Publ., 1990. 
[66] M. Ozawa. Quantum measuring processes of continuous observables. Journ. Math. Physics, 25:79-87, 1984.

[67] P. Panangaden. Labelled Markov Processes. Imperial College Press, 2009.

[68] S. Pulmannová and S. Gudder. Representation theorem for convex effect algebras. Commentationes Mathematicae Universitatis Carolinae, 39(4):645-659, 1998.

[69] H. Reichenbach. The theory of probability. Univ. California Press, 1949.

[70] M. Rennela. Towards a quantum domain theory: Order-enrichment and fixpoints in $W^{*}$-algebras. In B. Jacobs, A. Silva, and S. Staton, editors, Math. Found. of Programming Semantics, number 308 in Elect. Notes in Theor. Comp. Sci., pages 289-307, 2014.

[71] M. Rennela and S. Staton. Complete positivity and natural representation of quantum computations, 2015. Math. Found. of Programming Semantics XXXI.

[72] E. Rieffel and W. Polak. Quantum Computing. A Gentle Introduction. MIT Press, Cambridge, MA, 2011.

[73] W. Rudin. Functional Analysis. McGraw-Hill Book Company, 1987. Third, intern. edition.

[74] P. Selinger. Towards a quantum programming language. Math. Struct. in Comp. Sci., 14(4):527-586, 2004.

[75] S. Staton. Algebraic effects, linearity, and quantum programming languages. In Principles of Programming Languages, pages 395-406. ACM SIGPLAN-SIGACT, 2015.

[76] M. Stone. Postulates for the barycentric calculus. Ann. Math., 29:25-30, 1949.

[77] F. Strocchi. An Introduction to the Mathematical Structure of Quantum Mechanics: a short Course for Mathematicians, volume 27 of Adv. Series in Math. Physics. World Scientific, 2005.

[78] T. Swirszcz. Monadic functors and convexity. Bull. de l'Acad. Polonaise des Sciences. Sér. des sciences math., astr. et phys., 22:39-42, 1974.

[79] M. Takesaki. Theory of Operator Algebras I, volume 124 of Encyclopedia of Mathematical Sciences. Springer, $2^{\text {nd }}$ edition, 2001.

[80] M. Takesaki. Theory of Operator Algebras III, volume 127 of Encyclopedia of Mathematical Sciences. Springer, 2003.

[81] J. Tomiyama. On the projection of norm one in $W^{*}$-algebras. Proc. Japan Acad., 10:608-612, 1957.

[82] B. Valiron, N. Ross, D. Scott Alexander, P. Selinger, and J. Smith. Programming the quantum future. Communications of the ACM, 58(8):52-61, 2015.

[83] W. Wootters and W. Zurek. A single quantum cannot be cloned. Nature, 299:802-803, 1982.

\section{APPENDIX A.}

This appendix describes some background information for our running examples, involving discrete probability, continuous probability, Hilbert spaces, and $C^{*}$-algebras.

A.1. Discrete probability. To describe finite discrete probabilities categorically one uses the distribution monad $\mathcal{D}$ : Sets $\rightarrow$ Sets. It maps a set $X$ to the set $\mathcal{D}(X)$ of probability distributions over $X$, which we describe as formal finite convex sums:

$$
\sum_{i} r_{i}\left|x_{i}\right\rangle \quad \text { where } \quad x_{i} \in X \text { and } r_{i} \in[0,1] \text { satisfy } \sum_{i} r_{i}=1 .
$$

We use the "ket" notation $|-\rangle$ to distinguish elements $x \in X$ and their occurrences in formal sums. Alternatively one may describe $\mathcal{D}(X)$ as the set of functions $\varphi: X \rightarrow[0,1]$ with finite support and $\sum_{x} \varphi(x)=1$. We freely switch between these two descriptions. Each function $f: X \rightarrow Y$ gives a function $\mathcal{D}(f): \mathcal{D}(X) \rightarrow \mathcal{D}(Y)$, where:

$$
\mathcal{D}(f)\left(\sum_{i} r_{i}\left|x_{i}\right\rangle\right)=\sum_{i} r_{i}\left|f\left(x_{i}\right)\right\rangle .
$$

The unit $\eta: X \rightarrow \mathcal{D}(X)$ of this distribution monad $\mathcal{D}$ sends $x \in X$ to the singleton/Dirac distribution $\eta(x)=1|x\rangle$. The Kleisli extension $f_{*}: \mathcal{D}(X) \rightarrow \mathcal{D}(Y)$ of a function $f: X \rightarrow$ 
$\mathcal{D}(Y)$ is defined by:

$$
f_{*}(\varphi)(y)=\sum_{x} \varphi(x) \cdot f(x)(y)
$$

Like for any monad, one can form the Kleisli category $\mathcal{K} \ell(\mathcal{D})$. In this case we get the category of sets and stochastic matrices, as the objects of $\mathcal{K} \ell(\mathcal{D})$ are sets, and its maps $X \rightarrow Y$ are functions $X \rightarrow \mathcal{D}(Y)$. The unit function $\eta: X \rightarrow \mathcal{D}(X)$ is then the identity map $X \rightarrow X$ in $\mathcal{K} \ell(\mathcal{D})$. Composition of $f: X \rightarrow Y$ and $g: Y \rightarrow Z$ in $\mathcal{K} \ell(\mathcal{D})$ yields a map $g \odot f: X \rightarrow Z$, which, as a function $X \rightarrow \mathcal{D}(Z)$ is given by $g \circ f=g_{*} \circ f$, using Kleisli extension from (A.2).

There is a forgetful functor $\mathcal{K} \ell(\mathcal{D}) \rightarrow$ Sets, sending $X$ to $\mathcal{D}(X)$ and $f$ to $\mu \circ \mathcal{D}(f)$. It has a left adjoint $J$ : Sets $\rightarrow \mathcal{K} \ell(\mathcal{D})$ which is the identity on objects and sends $f$ to $\eta \circ f$. The Kleisli category $\mathcal{K} \ell(\mathcal{D})$ has coproducts $X_{1}+X_{2}$, on objects like in Sets. There are coprojection maps $J\left(\kappa_{i}\right)=\eta \circ \kappa_{i}: X_{i} \rightarrow \mathcal{D}\left(X_{1}+X_{2}\right)$, where $\kappa_{i}: X_{i} \rightarrow X_{1}+X_{2}$ are the coprojections in Sets. Cotuples in $\mathcal{K} \ell(\mathcal{D})$ are like in Sets. We have $\mathcal{D}(1)=1$ - making $\mathcal{D}$ an 'affine' functor. As a result, the singleton/final set $1=\{0\}$ is final in the category $\mathcal{K} \ell(\mathcal{D})$. The copower $n \cdot 1$ in $\mathcal{K} \ell(\mathcal{D})$ is thus the $n$-element set $n$.

The distribution monad $\mathcal{D}$ is 'commutative': there exists a natural transformation dst: $\mathcal{D}(X) \times \mathcal{D}(Y) \rightarrow \mathcal{D}(X \times Y)$, given by $d s t(\varphi, \psi)(x, y)=\varphi(x) \cdot \psi(y)$. It makes the monad 'monoidal'. Via this map dst the product $\times$ on Sets becomes a tensor on $\mathcal{K} \ell(\mathcal{D})$. Explicitly, dst is used for functoriality: for functions $f_{i}: X_{i} \rightarrow \mathcal{D}\left(Y_{i}\right)$ we can define a function $f_{1} \otimes f_{2}=d s t \circ\left(f_{1} \times f_{2}\right): X_{1} \times X_{2} \rightarrow \mathcal{D}\left(Y_{1} \times Y_{2}\right)$. The final object $1 \in \mathcal{K} \ell(\mathcal{D})$ is the unit for this tensor.

A.2. Continuous probability. We write Meas for the category with measurable spaces $X=\left(X, \Sigma_{X}\right)$ as objects, where $\Sigma_{X} \subseteq \mathcal{P}(X)$ is a $\sigma$-algebra. A measurable space $X$ is discrete if all subsets of $X$ are measurable, i.e. if $\Sigma_{X}=\mathcal{P}(X)$. A map $f: X \rightarrow Y$ in Meas is a 'measurable' function, satisfying $f^{-1}(M) \in \Sigma_{X}$ for each measurable subset $M \in \Sigma_{Y}$. We use the unit interval $[0,1]$ as measurable space, with 'Borel' measurable subsets generated by the intervals $[q, 1]$, where $q$ is a rational number in $[0,1]$.

The (discrete) empty and singleton spaces 0 and 1 are initial and final in the category Meas. The (categorical) product $X_{1} \times X_{2}$ of two measurable spaces $X_{i}$ carries the least $\sigma$-algebra making both projections $\pi_{i}: X_{1} \times X_{2} \rightarrow X_{i}$ measurable functions; equivalently, this $\sigma$-algebra is generated by the rectangles $M_{1} \times M_{2}$ with $M_{i} \in \Sigma_{X_{i}}$. The coproduct $X_{1}+X_{2}$ in Meas involves the disjoint union of the underlying sets with the $\sigma$-algebra given by the direct images $\kappa_{i} M=\left\{\kappa_{i} x \mid x \in M\right\}$ for $M \in \Sigma_{X_{i}}$, where $\kappa_{i}: X_{i} \rightarrow X_{1}+X_{2}$ is the coprojection map. Thus, if $X_{1}, X_{2}$ are discrete, then so is the coproduct $X_{1}+X_{2}$. In particular, the copower $n \cdot 1$ is the $n$-element discrete space.

A probability space consists of a measurable space $X=\left(X, \Sigma_{X}\right)$ together with a function $\phi: \Sigma_{X} \rightarrow[0,1]$ which satisfies $\phi(X)=1$ and is countably additive: $\phi\left(\emptyset_{i \in I} M_{i}\right)=$ $\sum_{i \in I} \phi\left(M_{i}\right)$, for each pairwise disjoint, countable collection of measurable subsets $M_{i} \in \Sigma_{X}$. Here we use $\oslash$ for disjoint union, where $\Sigma_{X}$ is understood as effect algebra. We write:

$$
\mathcal{G}(X)=\left\{\phi: \Sigma_{X} \rightarrow[0,1] \mid \phi \text { is a probability measure }\right\} .
$$

For each finite discrete space $n$ we have $\mathcal{G}(n) \cong \mathcal{D}(n)$. In particular, $\mathcal{G}(2) \cong[0,1]$ and $\mathcal{G}(1) \cong 1$.

Each measurable subset $M \in \Sigma_{X}$ yields a function $\mathrm{ev}_{M}: \mathcal{G}(X) \rightarrow[0,1]$, namely $\operatorname{ev}_{M}(\phi)=\phi(M)$. Thus one can equip the set $\mathcal{G}(X)$ with the least $\sigma$-algebra making all 
these maps $e_{M}$ measurable. We obtain the 'Giry' functor $\mathcal{G}:$ Meas $\rightarrow$ Meas from [28] (see also [55]): for a map $f: X \rightarrow Y$ in Meas we get a measurable function $\mathcal{G}(f): \mathcal{G}(X) \rightarrow \mathcal{G}(Y)$ given by:

$$
\mathcal{G}(f)\left(\Sigma_{X} \stackrel{\phi}{\rightarrow}[0,1]\right)=\left(\Sigma_{Y} \stackrel{f^{-1}}{\rightarrow} \Sigma_{X} \stackrel{\phi}{\rightarrow}[0,1]\right) .
$$

For a probability measure $\phi \in \mathcal{G}(X)$ we describe integration, but only for measurable functions (predicates) $X \rightarrow[0,1]$, with the unit interval as codomain, and not for more general real- or complex-valued functions (see [46] for a general effect-theoretic account). For each $M \in \Sigma_{X}$ we write $\mathbf{1}_{M}: X \rightarrow[0,1]$ for the indicator function given by $\mathbf{1}_{M}(x)=1$ for $x \in M$ and $\mathbf{1}_{M}(x)=0$ for $x \notin M$. The mapping $M \mapsto \mathbf{1}_{M}$ is a homomorphism of effect algebras form $\Sigma$ to the predicates on $X$, i.e. to the measurable functions $X \rightarrow[0,1]$. A step function is a finite linear combination $r_{1} \mathbf{1}_{M_{1}}+\cdots+r_{k} \mathbf{1}_{M_{k}}=\sum_{i} r_{i} \bullet \mathbf{1}_{M_{i}}$ of indicator functions with $r_{i} \in[0,1]$ and $M_{i} \in \Sigma_{X}$ pairwise disjoint measurable subsets. It is a basic fact that each measurable predicate $p: X \rightarrow[0,1]$ can be approximated (from below) by step functions: $p=\bigvee_{n \in \mathbb{N}} p_{n}$. The (Lebesgue) integral is then defined as:

$$
\int p \mathrm{~d} \phi=\bigvee_{n \in \mathbb{N}} \int p_{n} \mathrm{~d} \phi \in[0,1] \quad \text { where } \quad \int\left(\sum_{i} r_{i} \mathbf{1}_{M_{i}}\right) \mathrm{d} \phi=\sum_{i} r_{i} \phi\left(M_{i}\right) .
$$

Unit $\eta: X \rightarrow \mathcal{G}(X)$ and multiplication maps $\mu: \mathcal{G}^{2}(X) \rightarrow \mathcal{G}(X)$ can now be defined, making $\mathcal{G}$ a monad on the category Meas:

$$
\eta(x)(M)=\mathbf{1}_{M}(x) \quad \mu(\Phi)(M)=\int e v_{M} \mathrm{~d} \Phi .
$$

For details, we refer to [67] or [40, 46]. The Kleisli category $\mathcal{K} \ell(\mathcal{G})$ of the Giry monad contains measurable spaces as objects; a morphism $X \rightarrow Y$ in $\mathcal{K} \ell(\mathcal{G})$ is a measurable map $f: X \rightarrow \mathcal{G}(Y)$, which can be understood as a Markov transition map. There is an associated Kleisli extension map, which we write as $f_{*}: \mathcal{G}(X) \rightarrow \mathcal{G}(Y)$, namely:

$$
f_{*}(\phi)(N)=\int f(-)(N) \mathrm{d} \phi \quad \text { where } \quad f(-)(N): X \rightarrow[0,1] .
$$

Coproducts $(+, 0)$ are inherited from Meas. The final/singleton space 1 is also final in $\mathcal{K} \ell(\mathcal{G})$, since $\mathcal{G}(1)=1$. Further, the Giry monad is also commutative, like the distribution monad $\mathcal{D}$, via a map dst: $\mathcal{G}(X) \times \mathcal{G}(Y) \rightarrow \mathcal{G}(X \times Y)$ determined by $\operatorname{dst}(\phi, \psi)(M \times N)=\phi(M) \times \psi(N)$. As a result, the product $\times$ of measurable spaces becomes a tensor on the Kleisli category $\mathcal{K} \ell(\mathcal{G})$, with the final space 1 as tensor unit. For more information, see [67.

A.3. Hilbert spaces. A Hilbert space is a vector space (over $\mathbb{C}$ ) with an inner product such that the induced norm makes the space complete. We write Hilb for the category of Hilbert spaces, with linear and bounded (equivalently: continuous) maps between them. This category is symmetric monoidal, with the complex numbers $\mathbb{C}$ as unit for the tensor $\otimes$. There are also finite biproducts $(\oplus, 0)$, given by the cartesian product of the underlying space. Tensors $\otimes$ distribute over finite biproducts. Further, Hilb is a 'dagger category', where $f^{\dagger}$ is the conjugate transpose of a map $f$. A map $f$ is an isometry (aka. dagger monic) if $f^{\dagger} \circ f=\mathrm{id}$, and a unitary if not only $f^{\dagger} \circ f=$ id but also $f \circ f^{\dagger}=\mathrm{id}$. In that case $f^{\dagger}$ is the inverse of $f$. For a Hilbert space $\mathcal{H}$ we write $\mathcal{B}(\mathcal{H})$ for the set of endomaps $\mathcal{H} \rightarrow \mathcal{H}$ in Hilb. There is a subset $\mathcal{E} f(\mathcal{H})=\{A \in \mathcal{B}(\mathcal{H}) \mid 0 \leq A \leq$ id $\}$ of effects. Hilbert spaces will be used mostly as source of examples for $C^{*}$-algebras, via this $\mathcal{B}(-)$ construction. 
A.4. $C^{*}$-algebras. In the current context we use $C^{*}$-algebra over the complex numbers $\mathbb{C}$, with a multiplicative unit element 1 . Thus a $C^{*}$-algebra is a vector space over $\mathbb{C}$ which is *-algebra - with multiplication $\cdot$, involution $(-)^{*}$, and unit 1 - and carries a norm $\|-\|$ that makes the space complete and interacts with multiplication via $\|a \cdot b\| \leq\|a\| \cdot\|b\|$ and $\left\|a^{*} \cdot a\right\|=\|a\|^{2}$. A $C^{*}$-algebra is called commutative if its multiplication is commutative, and finite-dimensional if it has finite dimension when considered as a vector space.

An element $a \in A$ of a $C^{*}$-algebra $A$ is called self-adjoint if $a^{*}=a$ and positive if it is of the form $a=x^{*} x$ for some element $x \in A$. There is a partial order $a \leq b$ iff $b-a$ is positive.

A linear map $f: A \rightarrow B$ between $C^{*}$-algebras $A, B$ is called:

(1) multiplicative (M) if $f\left(a \cdot a^{\prime}\right)=f(a) \cdot f\left(a^{\prime}\right)$ for all $a, a^{\prime} \in A$;

(2) involutive (I) if $f\left(a^{*}\right)=f(a)^{*}$ for all $a \in A$;

(3) unital (U) if $f(1)=1$, and subunital ( $\mathrm{sU}$ ) if $0 \leq f(1) \leq 1$;

(4) positive (P) if $f(a) \geq 0$ for each $a \geq 0$;

(5) completely positive (CP) if for each $n \in \mathbb{N}$ the map $M_{n}(f): M_{n}(A) \rightarrow M_{n}(B)$ is positive, where $M_{n}(A)$ is the $C^{*}$-algebra of $n \times n$ matrices with entries from $A$, with matrix multiplication.

We call $f$ a MIU-map if it is multiplicative, involutive, and unital, and a (C)PU-map if it (completely) positive and unital. There are implications $\mathrm{MIU} \Rightarrow \mathrm{CPU} \Rightarrow \mathrm{PU} \Rightarrow \mathrm{I}$. A MIUmap is traditionally called a ${ }^{*}$-homomorphism. If either $A$ or $B$ is commutative, then a positive map $f: A \rightarrow B$ is automatically completely positive. We use categories of $C^{*}$-algebras Cstar $_{\mathrm{MIU}} \hookrightarrow$ Cstar $_{\mathrm{CPU}} \hookrightarrow$ Cstar $_{\mathrm{PU}}$ with maps as indicated by the subscripts. There are obvious subcategories $\mathbf{C C s t a r}_{\mathrm{PU}}$ and $\mathbf{C C s t a r}_{\mathrm{MIU}}$ of commutative $C^{*}$-algebras with $\mathrm{PU} / \mathrm{MIU}$ maps. The famous Gelfand duality involves an equivalence $\mathbf{C H} \simeq\left(\mathbf{C C s t a r}_{\mathrm{MIU}}\right)^{\mathrm{op}}$ where $\mathbf{C H}$ is the category of compact Hausdorff spaces and continuous maps. For PU-maps an equivalence $\mathcal{K} \ell(\mathcal{R}) \simeq\left(\mathbf{C C s t a r}_{\mathrm{PU}}\right)^{\text {op }}$ is shown in [26], where $\mathcal{R}$ is the Radon monad on CH.

The collection of endomaps $\mathcal{B}(\mathcal{H})$ of a Hilbert space $\mathcal{H}$ is a $C^{*}$-algebra, with multiplication given by composition. This mapping $\mathcal{H} \mapsto \mathcal{B}(\mathcal{H})$ gives rise to functors:

$$
\text { Hilb }_{\text {unit }} \stackrel{\mathcal{B}}{\longrightarrow}\left(\text { Cstar }_{\mathrm{MIU}}\right)^{\text {op }} \quad \text { Hilb }_{\text {isom }} \stackrel{\mathcal{B}}{\longrightarrow}\left(\text { Cstar }_{\mathrm{CPU}}\right)^{\text {op }}
$$

where $\mathbf{H i l b}_{\text {unit }}$, resp. Hilb $\mathbf{b}_{\text {isom }}$, is the category of Hilbert spaces with unitaries, resp. isometries, as morphisms. A map $f: \mathcal{H} \rightarrow \mathcal{K}$ is sent to $\mathcal{B}(f)=f^{\dagger}(-) f: \mathcal{B}(\mathcal{K}) \rightarrow \mathcal{B}(\mathcal{H})$.

The algebra $\mathbb{C}$ of complex numbers is initial among $C^{*}$-algebras, in all categories

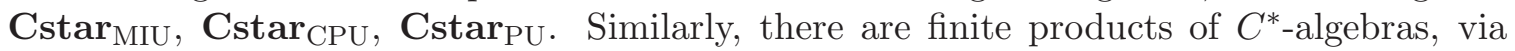
the trivial singleton space $\{0\}$ as final object, and via direct sums $\oplus$ of vector spaces (i.e. products of the underlying sets) as cartesian products. The operations are used pointwise. There are also tensor products of $C^{*}$-algebras. These are described in more detail in [79, section IV.4], but we outline them here. The $C^{*}$-tensors for two $C^{*}$-algebras $A$ and $B$ are obtained by taking the usual tensor of underlying vector spaces $A \otimes B$, defining a *-algebra structure as follows:

$$
\left(a_{1} \otimes b_{1}\right)\left(a_{2} \otimes b_{2}\right)=\left(a_{1} a_{2}\right) \otimes\left(b_{1} b_{2}\right) \quad(a \otimes b)^{*}=a^{*} \otimes b^{*} .
$$

One then obtains a $C^{*}$-algebra by introducing a $C^{*}$-norm compatible with the ${ }^{*}$-algebra structure, and taking the completion. There are minimal and maximal, or injective and projective $C^{*}$-norms, but if $A$ or $B$ is finite dimensional these coincide, see [80, chapter $\mathrm{XV}$, 
1.4-1.6]. Since $A \otimes B$ is the completion of the algebraic tensor of $A$ and $B$, the span of elements of the form $a \otimes b$ is dense, and in fact in the finite dimensional case, $A \otimes B$ is just the linear span of such elements.

The positive cone of $A \otimes B$ contains the positive elements according to the multiplication and involution, i.e. $a \in A \otimes B$ is positive if $a=x^{*} x$ for some other element $x$ in the tensor product. We note at this point that this cone is larger than the cone obtained by taking sums of elements $a \otimes b$ with $a \in A$ and $b \in B$ both positive. The effect of this is that no $C^{*}$-tensor is a functor on $\mathbf{C s t a r}_{\mathrm{PU}}$. Instead, the maps that can be tensored are the completely positive ones (see [79, §IV.3, and $\S I V .4$ Prop. 23]). In short, it is the category Cstar $_{\mathrm{CPU}}$ that is symmetric monoidal, with the (initial) object $\mathbb{C}$ as tensor unit. In [15] it is shown that the "minimal" tensor $\otimes$ on $\mathbf{C s t a r}_{\mathrm{CPU}}$ distributes over finite products $(\oplus,\{0\})$. We use this result in the form: the opposite category $\left(\mathbf{C s t a r}_{\mathrm{CPU}}\right)^{\mathrm{op}}$ is symmetric monoidal with the (final) object $\mathbb{C}$ as tensor unit, and $\otimes$ distributing over finite coproducts.

For $n \in \mathbb{N}$ one can write the matrix algebra $M_{n}(A)$ as tensor product $M_{n}(A) \cong M_{n}(\mathbb{C}) \otimes$ $A=\mathcal{B}\left(\mathbb{C}^{n}\right) \otimes A$. In particular, when $A$ is of the form $\mathcal{B}(\mathcal{H})$ we can move the tensor inside $\mathcal{B}(-)$ as in:

$$
\mathcal{B}\left(\mathbb{C}^{n}\right) \otimes \mathcal{B}(\mathcal{H}) \cong M_{n}(\mathcal{B}(\mathcal{H})) \cong \mathcal{B}\left(\mathcal{H}^{n}\right) \cong \mathcal{B}\left(\mathbb{C}^{n} \otimes \mathcal{H}\right),
$$

where $\mathcal{H}^{n}$ is the $n$-fold biproduct $\mathcal{H} \oplus \cdots \oplus \mathcal{H} \cong \mathbb{C}^{n} \otimes \mathcal{H}$.

A $W^{*}$-algebra (also called a von Neumann algebra) is a special kind of $C^{*}$ algebra that satisfies additional closure properties, see e.g. [79] for details. 